Pontifícia Universidade C $_{\text {atólica }}$

Marília Maurell Assad

\title{
Analysis of control strategies for autonomous scale motorcycles stabilization and trajectory tracking
}

Tese de Doutorado

Thesis presented to the Programa de PósGraduação em Engenharia Mecânica of PUC-Rio in partial fulfillment of the requirements for the degree of Doutor em Ciências - Engenharia Mecânica.

Advisor: Prof. Marco Antonio Meggiolaro

Rio de Janeiro

May 2018 


\section{Pontifícia Universidade Católica DO RIO DE JANEIRO}

Marília Maurell Assad

\section{Analysis of control strategies for autonomous scale motorcycles stabilization and trajectory tracking}

Thesis presented to the Programa de Pós-graduação em Engenharia Mecânica of PUC-Rio in partial fulfillment of the requirements for the degree of Doutor em Ciências Engenharia Mecânica. Approved by the undersigned Examination Committee.

Prof. Marco Antonio Meggiolaro Advisor Departamento de Engenharia Mecânica - PUC-Rio

Prof. Mauro Speranza Neto Departamento de Engenharia Mecânica - PUC-Rio

Prof. Helon Vicente Hultmann Ayala Departamento de Engenharia Mecânica - PUC-Rio

Prof. Fernando Ribeiro da Silva CEFET/RJ

Prof. Ricardo Alexandre Amar de Aguiar CEFET/RJ

Prof. Márcio da Silveira Carvalho

Vice Dean of Graduate Studies Centro Técnico Científico - PUC-Rio

Rio de Janeiro, May 21st, 2018 
All rigths reserved.

\section{Marília Maurell Assad}

Marília Assad graduated as a Control and Automation Engineer in 2010 from Pontifical Catholic University (Rio de Janeiro, RJ) and obtained her Master's degree in Mechanical Engineering in 2013 from the same institution.

Bibliographic data

Assad, Marília Maurell

Analysis of control strategies for autonomous scale motorcycles stabilization and trajectory tracking/ Marília Maurell Assad; advisor: Marco Antonio Meggiolaro. 2018.

131 f.: il. color; $30 \mathrm{~cm}$.

Tese (doutorado) - Pontifícia Universidade Católica do Rio de Janeiro, Departamento de Engenharia Mecânica, 2018.

Inclui referências bibliográficas.

1. Engenharia Mecânica - Teses. 2. Veículos autônomos. 3. Dinâmica de motocicletas. 4. Controle de estabilidade. 5. Controle de trajetória. 6. Ensino de engenharia. I. Meggiolaro, Marco Antonio. II. Pontifícia Universidade Católica do Rio de Janeiro. Departamento de Engenharia Mecânica. III. Título. 


\section{Acknowledgments}

First I would like to thank my family for all the support given through the development of this work and career choice.

To my advisor, Marco Antonio Meggiolaro, and professor Mauro Speranza for the guidance and support on the development of this thesis.

To my friends from graduate school and colleagues from LDSM (Laboratório de Desenvolvimento de Sistemas Mecatrônicos, in portuguese), who were not only indispensable to the construction of the test bench but also contributed with ideas and suggestions in moments of doubt.

To the Coordenação de Aperfeicoamento de Pessoal de Nivel Superior (CAPES) and to Conselho Nacional de Desenvolvimento Científico $e$ Tecnológico $(\mathrm{CNPq})$ for the scolarship and financial support to the research. To the Puc-Rio graduate course in Mechanical Engineering for the institutional support. 


\section{Abstract}

Assad, Marília Maurell. Analysis of control strategies for autonomous scale motorcycles stabilization and trajectory tracking. Rio de Janeiro, 2018. 131p. Tese de Doutorado - Departamento de Engenharia Mecânica, Pontifícia Universidade Católica do Rio de Janeiro.

Autonomous vehicles are an interesting and recent problem, with its application in cars and motorcycles still in its early stages. In addition to the inherent difficulties in making a vehicle move independently, the autonomous motorcycle has to be able to remain stable at any speed and trajectory. The vehicle's stability can be achieved by different solutions and control techniques. The main objective of this work is to develop an autonomous electric motorcycle with low cost sensing system. For this, a dynamic model of two-wheeled vehicles is analyzed, capable of describing the dynamic behavior while being simple enough to allow the implementation of real-time linear control strategies. The controller has two different objectives: to maintain the motorcycle stable and to follow a desired trajectory, in an autonomous way. Experiments were carried out with the small scale motorcycle aiming to characterize its elements for the theoretical model; then the proposed control strategies were simulated with the adjusted dynamic model. Finally, the control algorithms are applied to the real system through an actuated platform capable of reproducing the dynamic behavior of single-track vehicles. At last, the present work is a tool for teaching engineering, involving multilevel students around a complex, but familiar, problem. The system allows for continuous learning with increasing difficulty, involving multibody dynamics, experimental results analysis via software simulations, electronics and filters present in the embedded instrumentation and many control techniques to keep the system stable in every desired path, culminating in the experimental application of cited concepts.

\section{Keywords}

Autonomous vehicles; Motorcycle dynamics; Stability control; Trajectory control; Engineering education. 


\section{Resumo}

Assad, Marília Maurell. Análise de estratégias de controle para estabilização e acompanhamento de trajetórias de motocicletas autônomas em escala. Rio de Janeiro, 2018. 131p. Tese de Doutorado Departamento de Engenharia Mecânica, Pontifícia Universidade Católica do Rio de Janeiro.

Veículos autônomos são um problema recente, com aplicação em carros e motocicletas ainda nos estágios iniciais. Além das dificuldades inerentes de fazer um veículo mover-se independentemente, a motocicleta autônoma deve permanecer estável em qualquer velocidade e trajetória. O objetivo principal deste trabalho é desenvolver uma motocicleta elétrica autônoma com sistema de instrumentação de baixo custo. Para tanto, foi analisado um modelo dinâmico de motocicleta, capaz de reproduzir o comportamento real e permitindo a implementação de estratégias de controle linear em tempo real. O controlador tem dois objetivos diferentes: manter a motocicleta estável e seguir uma trajetória desejada, de forma autônoma. Experimentos foram realizados com a motocicleta de escala reduzida com o objetivo de caracterizar seus elementos; as estratégias de controle propostas foram simuladas com o modelo dinâmico ajustado. Por fim, os algoritmos de controle são aplicados ao sistema real através de uma plataforma atuada capaz de reproduzir a dinâmica de veículos de duas rodas. O presente trabalho é uma ferramenta para o ensino de engenharia, envolvendo estudantes de diferentes níveis em torno de um problema complexo. O sistema permite uma aprendizagem contínua com dificuldade crescente, envolvendo temas como dinâmica de multicorpos; análise de resultados através de simulações de software; eletrônica e filtros na instrumentação embutida e técnicas de controle para manter o sistema estável em todos os caminhos desejados, culminando na aplicação experimental dos conceitos citados.

\section{Palavras-chave}

Veículos autônomos; Dinâmica de motocicletas; Controle de estabilidade; Controle de trajetória; Ensino de engenharia. 


\section{Contents}

1 Introduction $\quad 15$

$\begin{array}{lll}1.1 & \text { Objective } & 15\end{array}$

$\begin{array}{lll}1.2 \text { Motivation } & 15\end{array}$

$\begin{array}{lll}1.3 & \text { Literature review } & 18\end{array}$

1.4 Original contributions 25

1.5 Thesis organization $\quad 27$

2 System characterization $\quad 28$

2.1 Data acquisition and control system 29

2.2 Inertial properties 30

$\begin{array}{lll}2.3 & \text { Traction system } & 37\end{array}$

$\begin{array}{lll}2.4 & \text { Steering system } & 40\end{array}$

3 Two-wheeled vehicle dynamics $\quad 44$

3.1 Linearized dynamic model 45

3.2 Analysis of the uncontrolled vehicle $\quad 50$

$4 \quad$ Control strategies $\quad 55$

4.1 Stability control through state feedback 56

4.2 Stability control through observer feedback 58

4.3 Path tracking through state feedback 62

4.4 Path tracking through linear quadratic regulator 65

$\begin{array}{lll}4.5 & \text { Path tracking through trajectory preview } & 67\end{array}$

$5 \quad$ State estimation through sensor fusion technique 78

$\begin{array}{lll}5.1 \text { Kalman filter algorithm } & 78\end{array}$

5.2 Motorcycle sensor fusion strategy 80

6 Actuated platform for motorcycle's simulation 90

6.1 Hardware in the loop 90

$\begin{array}{ll}6.2 \text { Actuated platform } & 91\end{array}$

$\begin{array}{lll}6.3 & \text { Experiments } & 94\end{array}$ 
7 Conclusions $\quad 98$

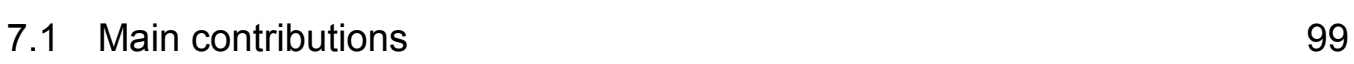

$\begin{array}{lll}7.2 & \text { Future works } & 100\end{array}$

$\begin{array}{lll}7.3 & \text { Thesis publications } & 101\end{array}$

$\begin{array}{ll}\text { Bibliography } & 103\end{array}$

Appendix A.Coefficients of the linearized two-wheeled model equations $\quad 117$

$\begin{array}{ll}\text { Appendix B.Traction motor characterization } & 121\end{array}$

$\begin{array}{ll}\text { Appendix C.IMU measurer } & 124\end{array}$

$\begin{array}{ll}\text { Appendix D.Programs } & 130\end{array}$ 


\section{List of Figures}

Figure 1.1 - Autonomous car Waymo and its instrumentation system. 16

$\begin{array}{ll}\text { Figure } 1.2 \text { - Yamaha's Motobot. } & 17\end{array}$

Figure 1.3 - Motorcycle C1, from Lit Motors, and its gyroscopic stabilizers. 17

Figure 1.4 - Honda's Riding Assist-e. 18

Figure 1.5 - Klein's rear-steered bicycle (Aström et al , 2005). 20

Figure 1.6 - Two-mass-skate bicycle (Kooijman et al, 2011). 20

Figure 2.1 - Small scale motorcycle, Duratrax DX450. 28

Figure 2.2 - Motorcycle with instrumentation system. 29

Figure 2.3 - Virtual model of motorcycle. 31

Figure 2.4 - Motorcycle's additional parameters. 31

Figure 2.5 - Motorcycle four main bodies. 32

Figure 2.6 - Experimental rig to measure center of mass. 32

Figure 2.7 - Center of mass measurement in x axis. 34

Figure 2.8 - Schematic of center of mass measurement in $\mathrm{x}$ axis. $\quad 34$

Figure 2.9 - Center of mass measurement in z axis. 35

Figure 2.10 - Schematic of center of mass measurement in $z$ axis. $\quad 35$

Figure 2.11 - Transmission system computational model. 38

Figure 2.12 - Transmission system test with 10\% step. 39

Figure 2.13 - Transmission system with 50\% step. 39

Figure 2.14 - Steering system experiment setup. $\quad 40$

Figure 2.15 - Potentiometer versus steering angle. 41

Figure 2.16 - Command signal versus steering angle. 42

Figure 2.17 - Steering system with 10\% step. 42

Figure 2.18 - Steering system with 40\% step. 43

Figure 3.1 - Motorcycle geometry main parameters. 46

Figure 3.2 - Global and local coordinate systems. 46

Figure 3.3 - Capsize mode, by <www.dynamotion.it>. 50

Figure 3.4 - Weave mode, by <www.dynamotion.it>. 51

Figure 3.5 - Wobble mode, by <www.dynamotion.it>. 51

Figure 3.6 - Regions of instability and self-stability of the motorcycle with forward speed increase. 53

Figure 3.7 - Motorcycle's poles values with forward speed increase. 53

Figure 4.1 - Block diagram for stability control through state feedback. 56 
Figure 4.2 - Angular speed and position results for stability control through state feedback.

Figure 4.3 - Torque input for stability control through state feedback. 58

Figure 4.4 - Block diagram for stability control through observer feedback. 58

Figure 4.5 - Angular speed and position results for stability control through observer feedback. 61

Figure 4.6 - Torque input for stability control through observer feedback. 61

Figure 4.7 - Block diagram for path tracking through output feedback. 62

Figure 4.8 -Position results for path tracking through state feedback. 64

Figure 4.9 - Torque input for path tracking through state feedback. 64

Figure 4.10 - Angular speed and position results for path tracking through state feedback. 64

Figure 4.11 - Position results for path tracking through LQR. 66

Figure 4.12 - Torque input for path tracking through LQR. 66

Figure 4.13 - Angular speed and position for path tracking through LQR. $\quad 67$

Figure 4.14 - Block diagram for roll angle control. 68

Figure 4.15 - Roll angles results for roll control through path preview. $\quad 69$

Figure 4.16 - Torque input for roll control through path preview. 69

Figure 4.17 - Angular speed and position results for roll control.

Figure 4.18 - Comparison between roll control strategies. 71

$\begin{array}{ll}\text { Figure } 4.19 \text { - Path preview schematic. } & 71\end{array}$

Figure 4.20 - Block diagram for trajectory control through path preview. 73

Figure 4.21 - Vehicle position and error in simulation for trajectory control $\begin{array}{ll}\text { through path preview. } & 74\end{array}$

$\begin{array}{ll}\text { Figure } 4.22 \text { - Torque input for trajectory control through path preview. } & 74\end{array}$

Figure 4.23 - Angular speed and position in simulation for trajectory control through path preview, at $5 \mathrm{~m} / \mathrm{s}$.

Figure 4.24 - Angular speed and position in simulation for trajectory control through path preview, at $10 \mathrm{~m} / \mathrm{s}$.

Figure 4.25 - Angular speed and position in simulation for trajectory control through path preview, at $15 \mathrm{~m} / \mathrm{s}$.

Figure 4.26 - Comparison between control strategies with the double lane change maneuver, at $10 \mathrm{~m} / \mathrm{s}$.

Figure 4.27 - Comparison between control strategies with the slalom maneuver, at $10 \mathrm{~m} / \mathrm{s}$.

Figure 5.1 - Motorcycle rotational planes. 
Figure 5.2 - Distance from IMU to origin point. 82

Figure 5.3 - Kinematic Kalman filter schematic. $\quad 85$

Figure 5.4 - Dynamical filter schematic. 86

Figure 5.5 - Concatenated Kalman filters schematic. 86

Figure 5.6 - Generated noisy data. $\quad 87$

Figure 5.7 - Kalman filter on the double lane change maneuver. 88

Figure 5.8 - Kalman filter on the slalom maneuver. 88

Figure 6.1 - Actuated platform concept. 92

Figure 6.2 - Actuated platform device. $\quad 92$

Figure 6.3 - Data flow on actuated platform. 93

Figure 6.4 - Real-time Labview interface. 93

Figure 6.5 - Roll control measurements from IMU. 94

Figure 6.6 - Simulation versus measurements for roll control. 95

Figure 6.7 - Trajectory and torque on path tracking simulation for platform. 96

Figure 6.8 - Angular speeds and positions on path tracking simulation for platform. $\quad 96$

Figure 6.9 - Path tracking measurements from IMU. $\quad 97$

Figure A.1 - Perpendicular distance $u$ (Sharp ,1971). 119

Figure A.2 - Axis in relation to frames. 120

$\begin{array}{ll}\text { Figure B.1 -Dynamometer for electric motors. } & 121\end{array}$

Figure B.2 -Dynamometer test with 10\% step. 122

Figure B.3 -Dynamometer test with 20\% step. 122

Figure B.4 -Dynamometer test with 30\% step. 123

$\begin{array}{ll}\text { Figure C.1 - IMU measurer. } & 124\end{array}$

Figure C.2 - Accelerations with planar position on the $x$ axis. 125

Figure C.3 - Comparison with encoder measurements, $x$ axis. 125

Figure C.4 - Accelerations with planar position on the $y$ axis. 126

Figure C.5 - Comparison with encoder measurements, $y$ axis. 126

Figure C.6 - Accelerations with planar position on the $z$ axis. 127

Figure C.7 - Comparison with encoder measurements, $z$ axis. 127

$\begin{array}{ll}\text { Figure C.8 - Inclined IMU position. } & 128\end{array}$

Figure C.9 - Accelerations with leaning position on the $x$ axis. 128

Figure C.10 - Comparison with encoder measurements, leaning position on the $x$ axis. 


\section{List of Tables}

Table 2.1 - Pixhawk technical features. 30

Table 2.2 - Motorcycle's parameters. 33

Table 2.3 - Experimental position of $x \boldsymbol{t}$. 34

Table 2.4 - Experimental position of $\boldsymbol{z t}$, with $\boldsymbol{h}=\mathbf{0 . 1 3 5} \mathbf{m}$. 36

Table 2.5 - Experimental position of $\boldsymbol{z t}$, with $\boldsymbol{h}=\mathbf{0 . 0 8 5} \mathbf{m}$. 36

Table 2.6 - Experimental position of $\boldsymbol{z t}$, with $\boldsymbol{h}=\mathbf{0 . 0 4 5} \boldsymbol{m}$. 36

Table 2.7 - Center of mass via software and experiments. 36

Table 2.8 - Traction motor technical features. 37

Table 2.9 - Transmission system. 38

Table 2.10 - Steering motor technical features. $\quad 40$

Table 4.1 - State feedback gain to three different speeds. 57

Table 4.2 - Observer feedback gain to three different speeds. 61

Table 4.3 - Path tracking state feedback gain to three different speeds. 63

Table 4.4 - Linear quadratic regulator gain to three different speeds. $\quad 65$

Table 4.5 - Roll control feedback gain to three different speeds. 69

Table 4.6 - Maximum error to path preview controller. 73 


\section{List of Symbols}

\section{Symbols}

\begin{tabular}{|c|c|}
\hline${ }^{j} a_{i}$ & Acceleration vector of body $i$ on reference $j$ \\
\hline$c_{i}$ & Cossine of angle $i$ \\
\hline$e_{p}$ & Path preview trajectory error \\
\hline$f$ & Ratio between mechanical trail and front wheel basis \\
\hline$F_{y}$ & Front wheel lateral reaction force \\
\hline$g$ & Gravity acceleration \\
\hline$I_{i_{a a}}$ & Moment of inertia of the body $i$ at axis $a$, in center of mass \\
\hline$J_{i a a}$ & Moment of inertia of the body $i$ at axis $a$, in reference system \\
\hline$K$ & Controller gain \\
\hline$m_{i}$ & Mass of the body $i$ \\
\hline$O$ & Coordinate system origin \\
\hline$r_{r w}, r_{f w}$ & Radius of rear and front wheel \\
\hline$r_{x}, r_{z}$ & IMU distance to system origin \\
\hline$R_{i}$ & Rotational matrix about axis $i$ \\
\hline$s_{i}$ & Sine of angle $i$ \\
\hline$S_{t}, S_{r}, S_{f}$ & Angular momentum of total system, rear wheel and front wheel \\
\hline$S_{u}$ & Static inertia term \\
\hline$t$ & Trail distance between steer axis and ground projection \\
\hline$T_{\delta}$ & Torque applied to the handlebar \\
\hline$T_{\varphi}$ & Torque applied to the rear frame \\
\hline$T_{p}$ & Time preview \\
\hline$u_{s}, u_{t}$ & PWM signal sent to servomotor and brushless motor, from 0 to 1 \\
\hline$u$ & $\begin{array}{l}\text { Perpendicular distance from front frame center of mass to the } \\
\text { steering axis }\end{array}$ \\
\hline$v_{x}$ & Speed of rear wheel along the $x_{r w}$ axis \\
\hline$x_{t}, z_{t}$ & Center of mass position \\
\hline$x_{r w}, y_{r w}, z_{r w}$ & Rear frame body coordinate system \\
\hline$X, Y, Z$ & Ground-fixed coordinate system \\
\hline$w$ & Distance between rear and front wheel \\
\hline
\end{tabular}




\section{Greek symbols}

$\delta \quad$ Motorcycle steering angle

$\epsilon \quad$ Front steering axis caster angle

$\xi \quad$ Integral of roll tracking error

$\tau \quad$ Torque

$\varphi \quad$ Rear frame roll angle at rear wheel referential

$\psi \quad$ Rear frame yaw angle at rear wheel referential

$\omega_{i} \quad$ Angular speed on axis $i$

\section{Superscript}

$\begin{array}{ll}\cdot & \text { First derivative } \\ - & \text { Second derivative } \\ \sim & \text { Estimated state } \\ B & \text { Predicted state } \\ R & \text { Reference system B } \\ T & \text { Reference system R } \\ & \text { Matrix transpose }\end{array}$

\section{Subscript}

$\epsilon$

$I M U$

Steering axis

IMU

Inertial Unit Measurement

$f f$

Front frame

$f w$

Front wheel

$m \quad$ Main body

$r f$

Rear frame

rw

Rear wheel

$s$

Steering body

$t \quad$ Total system (multibody motorcycle)

$x, y, z$

Axis 


\section{Introduction}

This chapter presents the main objectives, motivations and contributions of this work, as well as the organization of this document and literature review on autonomous two-wheeled vehicles.

\subsection{Objective}

The main objective of this work is to develop an autonomous electric motorcycle with low cost sensing system. To this end, a dynamic model of twowheeled vehicles is analyzed, which is capable of describing the real dynamic behavior while being simple enough to allow the implementation of real-time linear control strategies. The controller, in turn, has two different objectives: to maintain the motorcycle stable and to follow a desired trajectory, in an autonomous way.

Experiments were carried out with the small scale motorcycle aiming to characterize its elements for the theoretical model; then the proposed control strategies were simulated with the adjusted dynamic model. Finally, the control algorithms are applied to the real system through an actuated platform capable of reproducing the dynamic behavior of single-track vehicles.

\subsection{Motivation}

Autonomous vehicles - defined as land vehicles capable of transporting people or cargo without a human driver - are an interesting and recent problem. Though autopilot has been used on planes and ships since 1950, its application in cars and motorcycles is still in its early stages. Therefore, there is much to be developed in the area, taking into consideration that, in addition to being able to position itself globally and predict trajectories, there is an extra difficulty in dealing with obstacles and traffic signs - a common situation for a human drives, but a complex combination of sensors and control techniques for these autonomous systems. 
Since 2005, major automotive companies have started testing unmanned automotive systems. The most successful example so far is Waymo, previously known as Google's standalone car. Through embedded sensing and a robust control system, the equipment is capable of generating detailed threedimensional maps of its surroundings, dealing with obstruction on the track, tracing and identifying traffic signs such as speed limits, traffic lights and pedestrian tracks (Figure 1.1).

\section{HOW WAYMO'S SELF-DRIVING CAR WORKS}

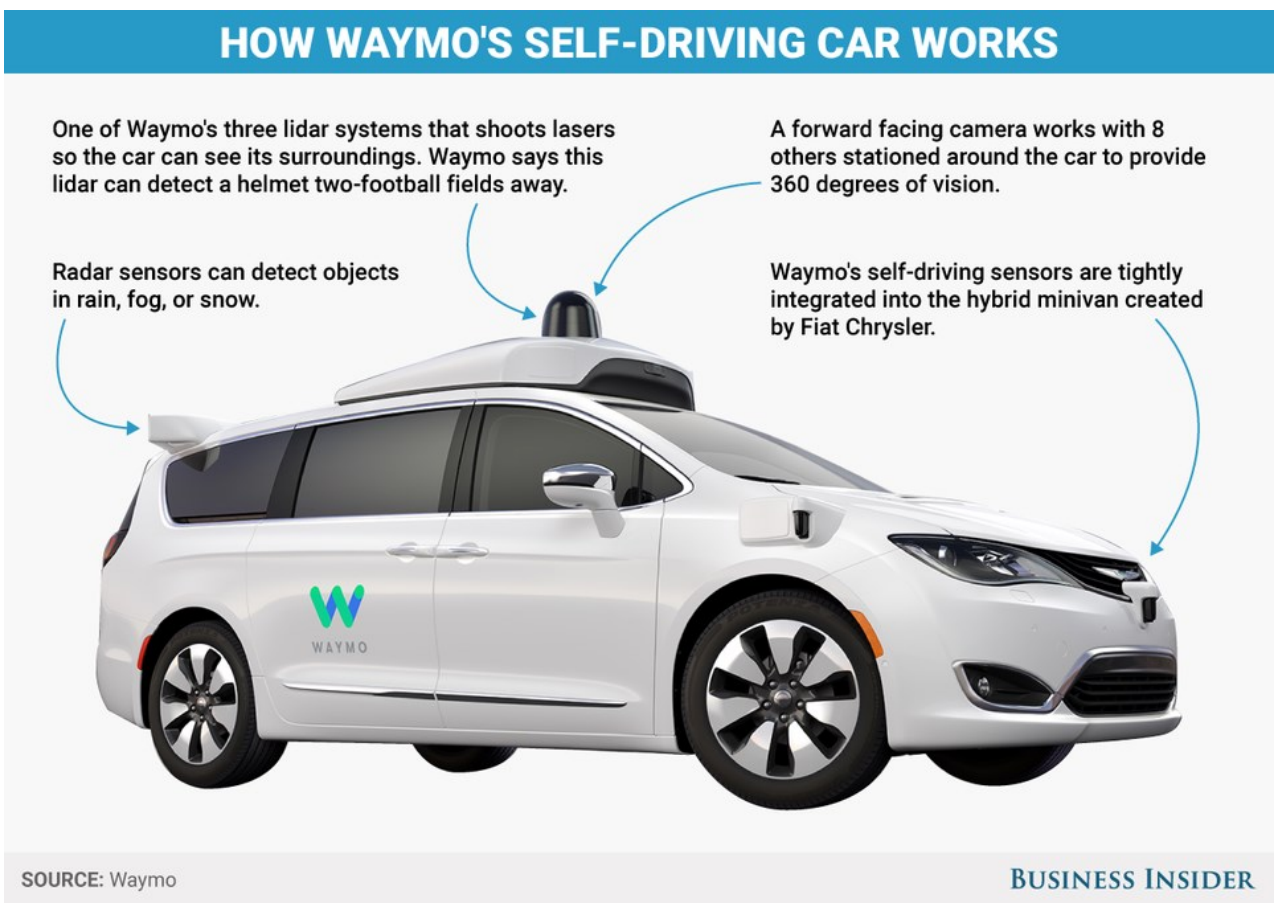

Source: <http://www.businessinsider.com/how-does-googles-waymo-self-driving-carwork-graphic-2017-1> Accessed in July, 2017.

Figure 1.1 -Autonomous car Waymo and its instrumentation system.

Regarding autonomous vehicles, another option less explored is the motorcycle. In addition to the inherent difficulties in making a vehicle move independently, the autonomous motorcycle has to be able to remain vertically stable at any speed and trajectory. Without steering or velocity control, singletrack vehicles tend to capsize, an additional problem that needs to be addressed by the autonomous control algorithm.

The industry has recently taken an interest in autonomous two-wheeled vehicles, with products such as the robot driver Motobot, from Yamaha (Figure 1.2). Its main purpose is to mimic a human rider, without making any fundamental modifications to the vehicle itself, knowledge that can be later applied to commercial motorcycles. In 2017, the robot was able to reach over $200 \mathrm{~km} / \mathrm{h}$ while autonomously driving on a racetrack. 


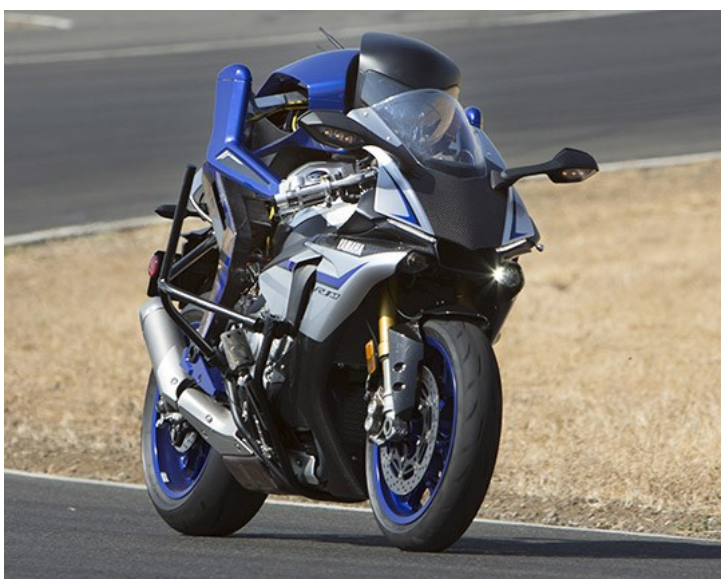

Source: <https://global.yamaha-motor.com/showroom/motobot/>. Accessed in October, 2017.

Figure 1.2 - Yamaha's Motobot.

Another solution to the roll stability problem of single-track vehicles, adopted by American company Lit Motors (Figure 1.3), is to use the controlled motion of masses, whose gyroscopic effect is capable of keeping the vehicle stable when moving, allowing it to lean itself into and out of turns and staying upright when stationary.

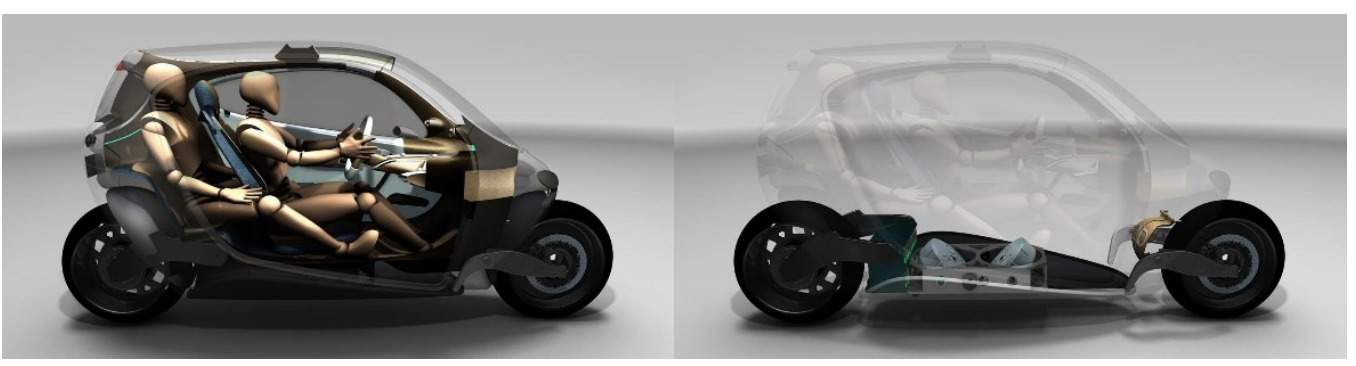

Source: <http://litmotors.com/c1/> Accessed January,2018.

Figure 1.3 - Motorcycle C1, from Lit Motors, and its gyroscopic stabilizers.

A third control alternative, implemented on Honda's Riding Assist-e, is to use the steer torque to balance the motorcycle (Figure 1.4). The system, not completely autonomous, aims to help inexperienced or inattentive pilots by steering the vehicle at low speeds, thus balancing itself while keeping a straight trajectory.

Therefore, the interest of the market and automotive industry in autonomous systems is proven. For the particular case of motorcycles, other factors make it particularly challenging and the forms of solutions to its inherent vertical instability problem are varied. Additionally, two-wheeled systems are a complex multibody system while being familiar to most students, which makes it a good candidate as a tool for teaching engineering. 


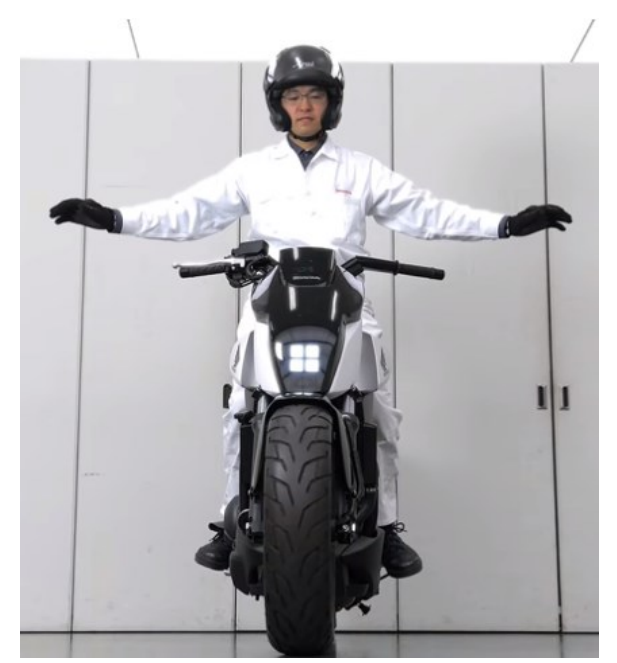

Source: < https://www.honda.com/mobility/riding-assist> Accessed February, 2017.

Figure 1.4 - Honda's Riding Assist-e.

\subsection{Literature review}

Several works in two-wheeled vehicles modeling and control - manned or unmanned - have been developed in order to overcome the problems inherent to this vertically unstable system. Regarding the mathematical modeling of the system, the most complete dynamical model of this type of vehicle is the nonlinear approach, such as the one developed in Yi et al (2009) and Zhang (2014). This model includes the complex tire-road interaction (Schiehlen, 2009), with tire deformation and slippage, and a constrained Lagrange modeling approach. The result, though physically more representative of a real motorcycle behavior, has greater complexity and implementation difficulty in a real-time control system.

The opposite strategy is to consider a linear model with reduced order, as Getz (1995): the wheels of the vehicle are negligible, providing only roll movement without side or longitudinal-slip and the frame is considered a point mass. This inverted-pendulum approach is interesting as a study object, to analyze the system stability or to simulate linear control strategies; however, the limitations of the model suggest a low practicality when applying the results on experimental single-track vehicles.

Multibody models bring a larger complexity to the system; the motorcycle is interpreted as a joint of rigid or flexible bodies, including the wheels, suspension, chassis, handlebar, fork and driver. Each body has a separate characteristic behavior, but when combined they influence the dynamics of one another. 
The main appeal of this method is the ability to add new elements while maintaining its linear characteristic, if necessary, or to be considered a junction of nonlinear components. The first approach results in lower order models which are, therefore, easier to simulate and implement control strategies (Aström et al, 2005).

A multibody linear second-order model can be achieved by assuming the vehicle is rolling on the horizontal plane, the rider has a fixed position and orientation relative to the frame, the forward velocity is constant and the steer axis is vertical. Increasing once again the complexity of this multibody model results in a linear fourth-order model, in which the static front fork model is replaced with a dynamic model; this is the Whipple bicycle model, developed in 1899 , and still currently used in two-wheeled vehicles studies.

Limebeer et al (2006) compared the differences between the cited approaches. The simpler inverted-pendulum models leads to conclude that steer angle and longitudinal speed are the only parameters that determine the lateral displacement, while self-stability characteristics are absent. The Whipple's model and its second order linearization are simple enough to be the study object of classical and modern control strategies; they also contain a sufficient level of physical realism, with complex interactions between its variables. At last, the nonlinear models provide insights into tire-road interaction, driver behavior, chassis flexibility, among other factors, at the expense of higher computational effort.

Due to the aforementioned advantages, this work uses the linear fourthorder model developed by Sharp (1971), a derivation of Whipple's bicycle model. The model considers four rigid bodies - two wheels, a rear frame with the rider rigidly attached to it and a front frame - characterized by 25 parameters (Meijaard et al, 2007). The model accounts for steer and roll torque inputs and has a general geometry and mass distribution that allows selfstability and uncontrolled steer dynamics effects.

Once the motorcycle model is defined, it is necessary to measure its parameters in order to guarantee realistic simulations. Kooijman et al (2008) and Moore et al (2010) present some simple experiments to determine the geometrical and mass parameters of a bicycle, analogue to motorcycles. The first author used a torsional pendulum to measure the mass moments of inertia; the second author repeated the same tests to eight different bicycles, with consistent results, which corroborates the method efficiency. 
It is interesting to analyze how the design parameters influence the maneuverability and stability of two-wheeled vehicles. Tak et al (2010) concludes that head (or camber) angle is the most significant parameter for the bicycle stability, followed by other front frame elements. Aström et al (2005) and Limebeer et al (2006) have expanded this analysis with unusual theoretical bicycles prototypes, such as the rear-steered model (Figure 1.5). Kooijman et al (2011) built an experimental two-mass-skate bicycle (Figure 1.6), capable of self-stability without trail. In summary, these works conclude that the stability of two-wheeled vehicles is affected by two dominant phenomena: a destabilizing gravity torque and the linear and angular acceleration due to steering.

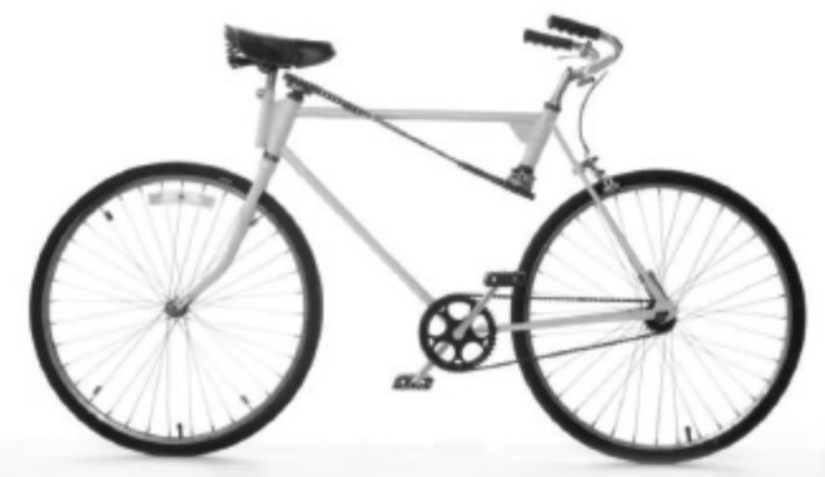

Figure 1.5 - Klein's rear-steered bicycle (Aström et al , 2005).

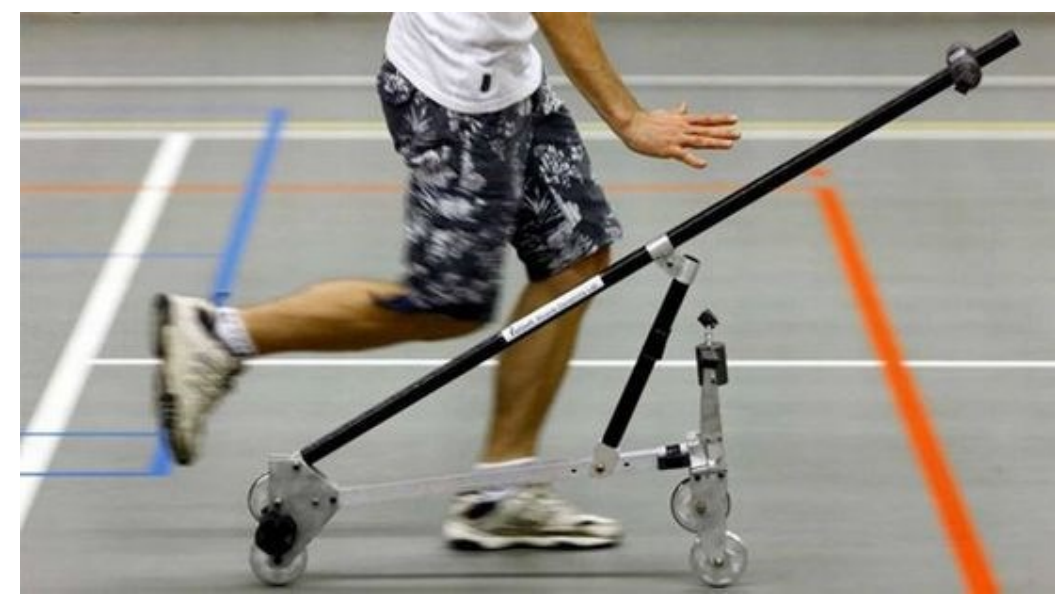

Figure 1.6 - Two-mass-skate bicycle (Kooijman et al , 2011).

The precession of the front wheel has been thoroughly studied by many authors; Lowell and McKell (1982) argue that this gyroscopic torque contributes to the stability of the vehicle by balancing the lean caused by perturbations with a centrifugal force. On the other hand, the gyroscopic effect makes the front wheel direction more oscillatory, since steering corrections result in new perturbations to the vehicle motion. 
A second interesting phenomenon that happens with the steering system is the counterintuitive need to turn the handlebar initially right when making a turn to the left. This countersteering produces a centrifugal force which leans the motorcycle frame to the opposite side - the initially intended turn side similar to the inverted-pendulum behavior; once the centrifugal and gravitational torques balance, the lean angle reaches a steady state and the vehicle can be steered to the original intended direction (Fajans, 2000). The same result can be obtained from throwing the hips in the direction counter to the turn, strategy largely used by racing motorcycle riders at high speeds.

A third well known phenomenon on motorcycles is the wobble effect, a spontaneous steering oscillation of the front wheel, which usually builds as the speed approaches a certain threshold (Ringwood and Feng, 2007). Ott et al (2010) analyzed the influence of cornering stiffness of the front tire, handling grip of rider and steering assembly on wobble.

Finally, once the vehicle is completely defined by mathematical models, the missing element to the system is the rider. Much like the dynamical twowheeled vehicle model, the driver can be modeled with more or less degrees of freedom, depending on the rider control outputs expected. The simpler definition is to consider the driver a rigid extension to the rear frame, while more complex analysis can model it as an inverted pendulum with one or two joints allowing upper body and hip movements - or even a separate multi-body with multiple links.

Kooijman (2012) does an extensive study on rider modeling through the multi-body approach, with experimental observation of riders on a treadmill and on the road. The work has concluded that human rider predominantly uses steering actions to stabilize the bicycle in favor of leaning control motions, being easily described by a rigid extension of the rear frame. Lange (2011) opts for a different approach, by modelling the driver as a black box transfer function, whose parameters were tuned by comparison with experimental data from human drivers.

Regarding the motorcycle stability control, authors are divided between two strategies: to control the vehicle steering the handlebar or through a physical balancer, imitating the inverted double pendulum effect caused by human drivers. The advantage of the second strategy is the possibility of remaining stable even at zero speeds, though Zhang et al (2011), unlike commonly expected, have proven a stationary riderless motorcycle can maintain its balance within a certain dynamic region only by steering. 
The balancer controllers appears in many different settings: Beznos et al (1998) and the previously cited C1 prototype, from Lit Motors, use two coupled gyroscopes spinning in opposite directions; Gallaspy and Hung (1999) mounted a disc, spinning around the vertical axis, on the top of a bicycle. Yamakita et al (2006) utilized a balancer - an actuated rod - whose behavior resembles more a human driver's upper body. Keo et al (2011) compared this controller to the flywheel approach, in which a spinning rod functions as a gyroscope, and concluded the balancer has a poorer performance stabilizing the vehicle and coping with larger disturbances, though it can perform more dynamic motions by changing the motorcycle's center of gravity position.

The steering control takes advantage of the aforementioned countersteering phenomenon: in order to yield a lean movement without an actuated pendulum, the vehicle is initially steered to the opposite desired direction, causing a centrifugal force that tilts the motorcycle in the desired direction. Aström and Murray (2010) present a simple linear steering controller, with the steer angle being proportional to the roll angle; Tanaka and Murakami (2004) develop a linear second order steering controller while Yi et al (2006) are able to keep the motorcycle upright, even when stationary, with a nonlinear balancing controller. As the objective of this work is to develop a small scale autonomous motorcycle, the steering control method is adopted in detriment of the balancer controller.

Regarding the trajectory control, one of the most applied strategies is the path preview control, in which the system analyzes a single-point at a predefined distance ahead and adjust the vehicle's path accordingly. Largely used in autonomous four-wheeled vehicles, this technique needs an adaptation to single-track systems: the result of the path tracking controller is the input to a roll angle controller, guaranteeing the stability of the vehicle at any trajectory (Getz and Marsden, 1995). Dao and Chen (2011) and Schmitt et al (2009) use a full state feedback controller to achieve this goal, while Sharp (2007) simulates an optimal linear controller. Yi et al (2006) and Zhang et al (2011) use a nonlinear model to correct the actual vehicle's path into the next desired trajectory point and to keep the equilibrium of the internal state, the roll angle.

Other path tracking control strategies are worth mentioning, such as the reinforcement learning proposed by Randlov and Alstrom (1998), in which a neural network is trained to balance a bicycle and to drive to a specific place. Chen and Dao (2007) developed a PID controller for the roll angle equilibrium and a fuzzy controller for the path tracking problem - which is able to minimize 
the distance between actual and desired position without using dynamic models as a basis - whose parameters are optimized via a genetic algorithm. Frezza et al (2004) present a model predictive controller, where the steering control action is calculated by considering the roll angle as an input and inverting the system's dynamics.

The control algorithms need information on the current motorcycle state to take the most appropriate actions. Since it is costly and inefficient to use a sensor for each state variable, the most interesting approach is to use a parameter estimation technique. Chenane et al (2012), for example, implemented a proportional two-integral observer capable of estimating the steering angle, its disturbance and the state variables (yaw and roll angular speeds, longitudinal velocity and roll angle) based on the linear dynamical motorcycle model.

However, the most widespread estimation technique is the Kalman filter, due to its ability to merge information from different sensors while simultaneously filtering measurement uncertainties. Additionally, since the Kalman filter design depends on the considered model, it can be easily adjusted to the available sensors and desired estimated variables. Zhang et al (2013) applies an extended Kalman filter to merge the data from inertial measurement units in the bicycle and rider with force sensors on the seat, analyzing both the vehicle's and driver states, as well as their interaction.

Corbetta et al (2010) compares the performance of an unscented and extended Kalman filter when estimating the motorcycle roll angle through inertial measuring unit and electro-optimal triangulators on the side of the vehicle; Boniolo et al (2008), on the other hand, proposes to obtain the same result by using only gyrometers and a wheel encoder, and having two Kalman filters - one for high-frequency signals and one for low-frequency signals, processed via static relationships. Tanelli et al (2014) expands the former approach by adding a vehicle speed estimation filter, considering the wheel slip and fusing data from the wheel encoder and longitudinal accelerometer.

Schilipsing et al (2012) also developed a filter to estimate a motorcycle's roll angle, comparing the performance of an extended Kalman filter via inertial measurement unit with a purely video-based filter, concluding that each filter produce errors in different situations and a fusion of both methods can yield improvements. 
The Kalman filter can also estimate the two-wheeled vehicles position. Gasbarro et al (2004) reconstruct a motorcycle trajectory by merging images from an on-board camera and MEMS accelerometers data; Guarnieri et al (2013) does the same through a cascade of a Kalman filter and Bayesian particle filter. Teerhuis and Jansen (2012) provide a more complete extended Kalman filter, estimating the motorcycle position and orientation via the data fusion of five sensors (steering angle encoder, inertial measurement unit, GPS, front and rear wheel speed sensors).

At last, two-wheeled vehicles are an interesting study object, since it can be presented as a simple model to undergraduate students to a complex multibody system to advanced classes. Besides, bicycles are a familiar system to students, so they become a concrete example of a dynamic system that uses a full range of systems-theoretic tolls so as to be challenging, but not overpowering (Klein, 1989).

Starting at the analysis of the dynamic model, students can learn to interpret a wide variety of concepts such as open-loop stabilization; the role of poles and zeros - especially in the right-half plane; the importance of a good design through parametric influences, as altering mass distribution or damping constants; physical limitations like maximum torque supplied by driver or motor.

An additional parameter, inherent only to motorcycles, is the in-plane dynamics, in which the suspension system contributes to the vehicle stability. Though not explored in this work, this feature provides the opportunity of discussing frequency domain characteristics with the students, as presented in Cossalter (2006), Tanelli et al (2014) and Speranza Neto et al (2017).

The next step is to simulate the expected results in a virtual ambient. Escalona and Recuero (2010) created an interactive real-time simulator using Matlab/Simulink and the non-linear equations of a bicycle, which helps engineering students to visualize the practical application of the previous computational analysis. Frezza et al (2004) broaden this concept by adding a driver to the simulation environment, allowing the reproduction of different control strategies.

Evolving into the physical world, hardware-in-the-loop simulators merge dynamical simulations with real-time visual or physical responses, creating a controlled environment for reproducing dynamic behaviors or analyzing human responses in certain conditions. Schwab and Recuero (2013) developed a bicycle simulator using a haptic feedback handlebar and visual simulation; Tanelli et al (2014) built a full motorcycle mock-up with sensorized handlebar 
and footpads, and fourteen degrees of freedom - including the reproduction of the vehicle's main motions, such as lateral displacement, yaw, roll, pitch and steer rotations, all supplemented by a virtual scenario.

Through a multidisciplinary approach, the two-wheeled vehicle is also a good study object, involving the previously cited multibody dynamics, electronics and filters present in the embedded instrumentation and many control techniques to keep the system stable in every desired path. The system allows for continuous learning with increasing difficulty, since the students start with identifying the physical parameters, progress to analyze its geometry and characteristics through computer software, test their own control strategy with the help of computational simulations and, at last, apply the result in the real system (Åström, 2005).

This problem-based learning technique can involve multilevel students around one single and very complex problem, as advocated by Martins (2016). Undergraduate students from the first semester can become familiar with simple instrumentation tasks such as collecting data from inertial measurement units via low cost microcontrollers like Arduino, while more advanced classes may apply complex techniques such as modern control or sensors fusion to the same system. Additionally, students can develop different side projects on the same theme (Assad and Speranza Neto, 2017); these devices can be used for testing, simulating and optimizing prototypes, as well as engaging students to combine practical activities with theory.

\subsection{Original contributions}

As will be exposed in Chapter 3, the dynamic modelling of two-wheeled vehicles has intrigued scientists since the nineteenth century, with the Whipple model. It has gained greater repercussion since the seventies, with the increase of ecological consciousness and the search for alternative transports.

The effort to characterize the dynamic model of single-track vehicles is notorious; Kooijman (2012) presented a literature review on the subject, starting with the Whipple model - the first equation of motion for a bicycle that could predict instability modes - up to the multibody approach (Meijaard, 2007), which demands the aid of computational programs.

Other works develop different strategies, from simple transfer functions (Getz, 1995), to state space models (Aström et al, 2005) and nonlinear approaches (Yi et al, 2009; Zhang, 2014). Thus, it was not the scope of this 
work to develop a new multibody model for motorcycles; the contribution of this thesis is to apply this widespread knowledge in a small scale system and verify its representativeness on this type of vehicle.

As shown in Chapter 2, the use of model building technology is noteworthy, as previous works studied ridable bicycles or motorcycles. One exception (Beznos et al, 1998) employed a gyroscopic stabilization in a small bicycle with front wheel drive, which cannot be associated to conventional twowheeled vehicles - due to major differences in its design - as opposed to the small scale electric motorcycle analyzed in this work.

Additionally, the pieces of equipment developed to characterize the scale system - namely, the center of mass measurer, IMU measurer and dynamometer - also use model building technology, being interesting study objects for engineering teaching. Klein (1989) and Aström et al (2005) reinforced the importance of using familiar systems as study objects to engage students' curiosity; this work contributed with three scale low-cost equipment, which are easily manipulated by students with different levels and could be reused in other projects.

Still focusing on using this thesis as an educational object, the control strategies developed in this work present an increasing complexity, in order to engage students. Regarding the stability control, previous works have studied linear control approaches such as proportional-integration-derivative feedback (Aström and Murray, 2010; Tanaka and Murakami, 2004), though not with the full state feedback, as developed here. This work also contributed with the use of a minimum-order observer to estimate the variables not directly measured by sensors.

As for the trajectory control strategies, Schmitt et al (2009) developed a state feedback controller by shifting the unstable poles to the stable region of the s-plane; the system response is then dependent on the longitudinal speed of the system. This work contributed to this modern control approach by aiming to guarantee the same dynamic behavior independently of the speed, with the same desired eigenvalues for the pole placement technique. Another addition to the literature is the linear quadratic regulator controller applied to the linear model using exclusively the steering torque input.

The sensor fusion strategy based on the use of low cost inertial measurement units brings economic advantages in the instrumentation costs and innovation, in the sense that previous works (Boniolo et al, 2010; Zhang, 2014) use the fusion of accelerometers and gyrometers to determine only the 
roll and pitch angle of the vehicle. This work, on the other hand, contributed to the study of the use of Kalman filters with the previous two sensors to additionally obtain the motorcycle position in the plane, without the aid of supplementary devices such as GPS, computer vision or external locators.

Finally, this work developed a hardware-in-the-loop simulator, such as the ones built by Schwab and Recuero (2013) or Tanelli et al (2014). The scale actuated platform, besides aiding to visually verify the control and sensor fusion strategies implemented, may also serve as a teaching object to several undergraduate and graduate students, in the Mechanical and Control and Automation fields. The experimental bench is complex enough to allow three degrees of freedom motions, which can represent several vehicles, such as cars, bicycles or ships, while its graphic and real visualization simplifies to the students the dynamics happening in the background.

\subsection{Thesis organization}

Chapter 2 describes the experimental study object, its components and sensors, as well as the tests made to characterize the system. Chapter 3 brings the multibody linear model description - with greater detail in Appendix A - and a self-stability analysis. In Chapter 4 , five control strategies are analyzed and simulated with the state space model adjusted to the experimental motorcycle. Chapter 5 describes a data fusion technique, the Kalman filter, applied to the system; an additional experiment test its validity, the IMU measurer. Finally, Chapter 6 presents a new actuated platform, built to simulate single-track vehicles dynamics as a risk-free environment to test the proposed control strategies. Chapter 7 brings suggestions for future work and the conclusions of the thesis. 


\section{System characterization}

In order to verify the validity of the proposed mathematical model and control strategies, a small-scale two-wheeled system is used as test rig. This chapter presents the motorcycle's experimental characterization, including its main geometric and inertial characteristics, traction motor, steering system and embedded instrumentation. The vehicle studied in this work, represented in Figure 2.1 , is a 1:5 scale motorcycle, propelled by a brushless motor, steered by a servomotor and originally radio-controlled.

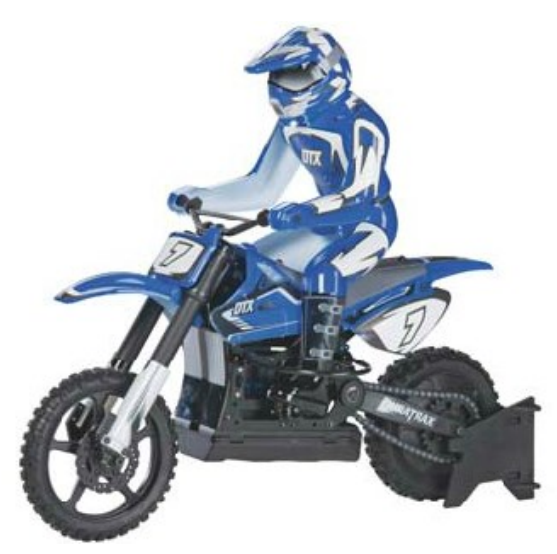

Figure 2.1 - Small scale motorcycle, Duratrax DX450.

An instrumentation system - consisting of a tachometer on the rear wheel and a potentiometer on the handlebar - and microcontroller were embedded to measure the motorcycle's linear and angular position, speed and acceleration, while guarantying its stability and trajectory (Figure 2.2). The main microcontroller is responsible for collecting the multiple sensors data, executing the control algorithm and applying the corrective signals to both electric motors, ensuring the vehicle's stable path.

The main advantages of using a scale model as study object are the easiness for transportation and visualization in classrooms, reduced workspace and low cost, since most of its components can be found in the accessible model building market. In the next sessions, the specifications and calibration tests of each component are further detailed. 


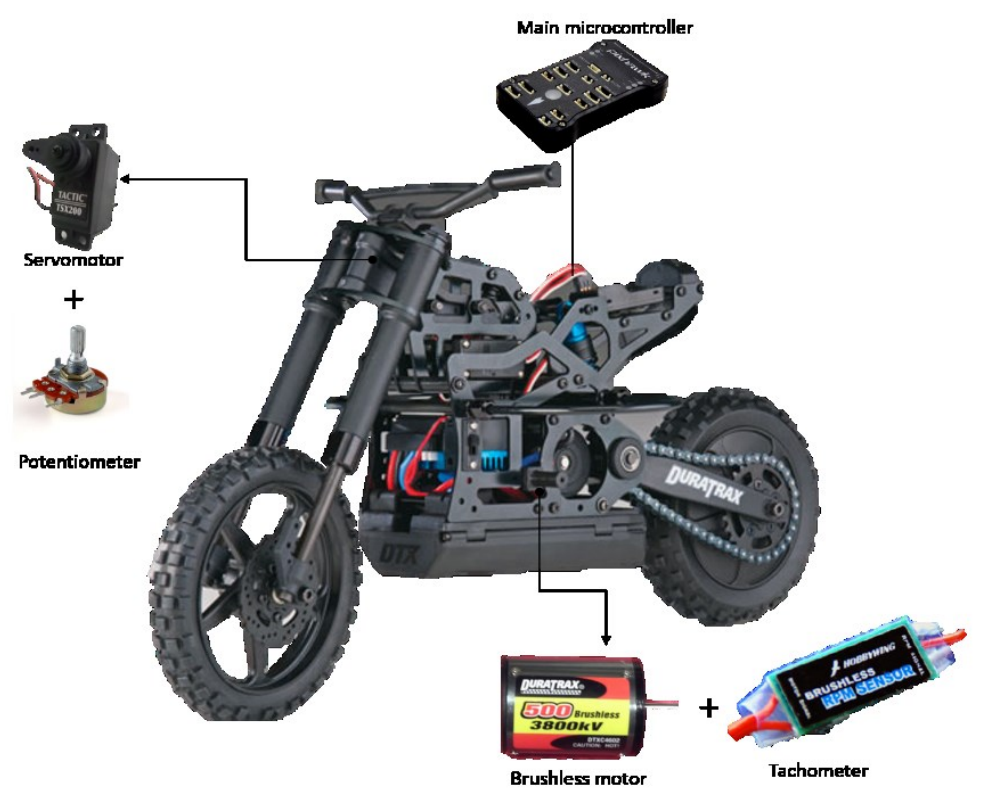

Figure 2.2 - Motorcycle with instrumentation system.

\subsection{Data acquisition and control system}

The system has two microcontrollers: one is responsible for collecting information from the instrumentation system and the other makes the necessary calculations and applies the result of the control algorithm to the motorcycle's speed and steer angle. The first task is accomplished by an Arduino Nano, which is exclusively dedicated to count the tachometer's number of pulses in a certain time frame in order to calculate the motor's angular speed in rpm, as well as to obtain the resistance value of the potentiometer; both information are then sent via serial communication to the main controller.

The second task is executed by the main controller, a Pixhawk board, whose main features are at Table 2.1. Its principal advantage is having the main sensors needed for autonomous navigation already embedded, allied with a good processing capacity and lightweight. Additionally, the board has a radio telemetry system that allows for real time data analysis via computer, and a $S D$ card system, for post-processing analysis. With a multithreading architecture in $C$ language, the board is capable of managing simultaneously the sensor's data acquisition - from both the Arduino board and the embedded sensors, sensor fusion and feedback control techniques.

The Pixhawk board has five sensor modules, a gyrometer, accelerometer, magnetometer, barometer and GPS. Only the first two sensors were used in this work: the magnetometer's reading was highly influenced by the electric brushless motor, being no longer able to correctly identify the Earth's magnetic 
field; the barometer measures altitude from different atmospheric pressures, which is only interesting in flying vehicles; finally, the GPS module does not work indoors, where all the tests of this work were done.

The first sensor module consists of a gyrometer, capable of measuring the angular velocities on three axes; this MEMS (MicroElectroMechanical System) equipment measures the vibration of objects under a rotational effect, with a maximum range of 2000 degrees per second. The second module contains an accelerometer and a magnetometer, both capable of sensing linear accelerations and the Earth's magnetic field on three axes, at a maximum range of 16 times the gravity acceleration and 12 gauss; the third module used contained another set of three axe gyrometer and accelerometer, for redundancy.

Table 2.1 - Pixhawk technical features.

\begin{tabular}{|c|c|c|}
\hline \multicolumn{2}{|l|}{ Manufacturer } & 3DRobotics \\
\hline \multicolumn{2}{|l|}{ Weight } & $39[\mathrm{~g}]$ \\
\hline \multicolumn{2}{|l|}{ Dimensions } & $50 \times 15,5 \times 81,5[\mathrm{~mm}]$ \\
\hline \multicolumn{2}{|c|}{ Telemetry frequency } & $915[\mathrm{MHz}]$ \\
\hline \multirow{3}{*}{ Sensor modules } & ST Micro L3GD20 & 3-axis gyrometer \\
\hline & ST Micro LSM303D & $\begin{array}{l}\text { 3-axis accelerometer } \\
\text { 3-axis magnetometer }\end{array}$ \\
\hline & Invensense MPU 6000 & $\begin{array}{l}\text { 3-axis gyrometer } \\
\text { 3-axis accelerometer }\end{array}$ \\
\hline
\end{tabular}

\subsection{Inertial properties}

The Duratrax DX450 motorcycle has been completely disassembled and all parts have been measured and weighed so that the virtual model represents accurately the real system. Figure 2.3 shows the final result in SolidWorks; the vehicle's center of mass and inertial moments were then calculated through the computational model and later verified through experiments. 


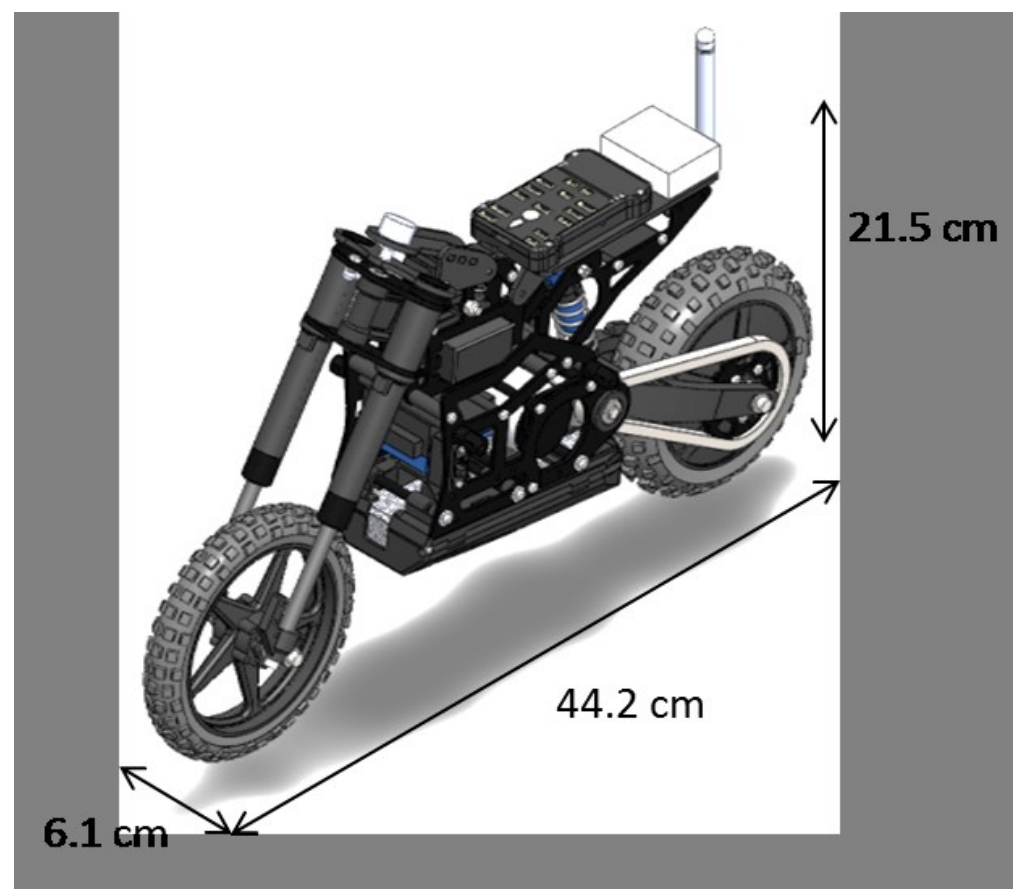

Figure 2.3 - Virtual model of motorcycle.

The system was divided in four main bodies: the front and rear wheel, the steering system and the main body (Figure 2.5), and the end result is in Table 2.2, with additional parameters explicit on Figure 2.4, such as the distance between wheels $(w)$, caster angle $(\epsilon)$, trail distance $(t)$ and approximate center of mass for the motorcycle $\left(x_{t}, z_{t}\right)$. Appendix A explicits how those parameters are combined in order to create the linearized two-wheeled vehicle model. It is interesting to note that the virtual position of the whole vehicle's center of mass is close to zero on the $y$ axis, which corroborates the hypothesis of the motorcycle being symmetrical on this particular axis.

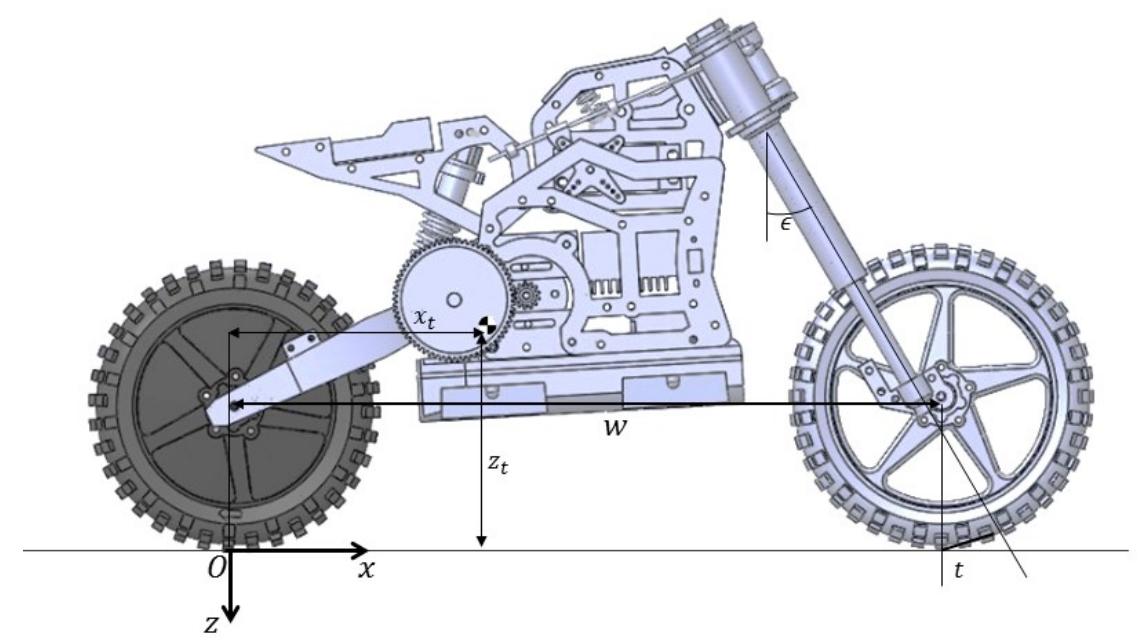

Figure 2.4 - Motorcycle's additional parameters. 


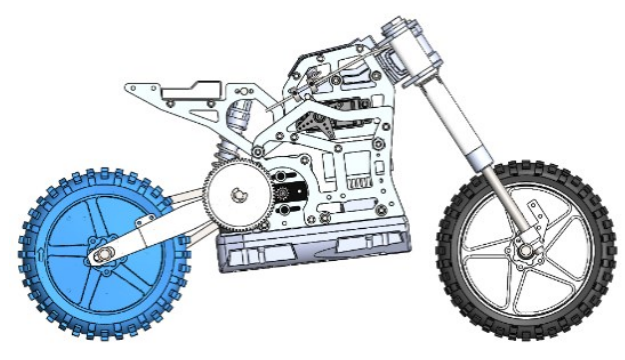

(a)

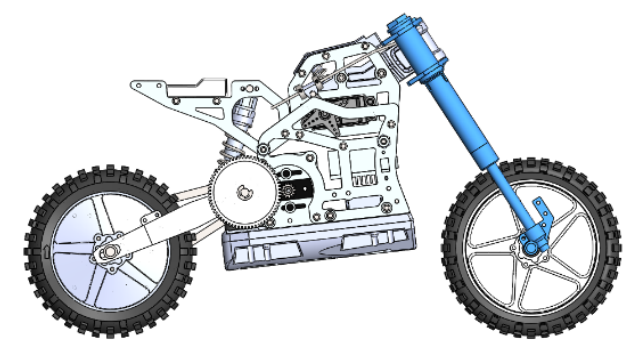

(c)

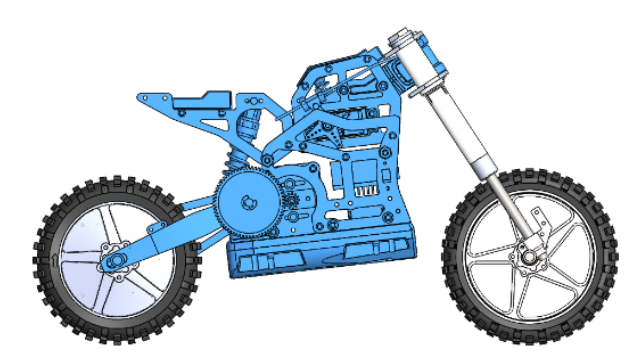

(b)

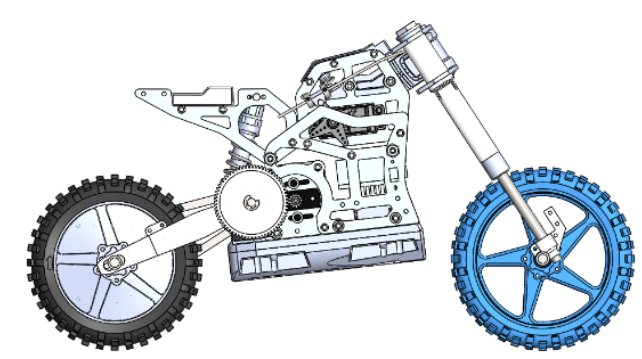

(d)

Figure 2.5 - Motorcycle four main bodies: (a) Rear wheel; (b) Main body;

(c) Steering body; (d) Front wheel.

In order to validate the motorcycle's inertial and geometric properties calculated via software, a simple experiment was used to obtain the vehicle center of mass. The apparatus consisted of two scales (TrackStar Corner) capable of measuring up to $2 \mathrm{~kg}$ with $0.5 \mathrm{~g}$ of uncertainty; the test rig is free to move in the $x$ and $z$ axis, i.e., both scales have variable distance and height, as exposed in Figure 2.6.

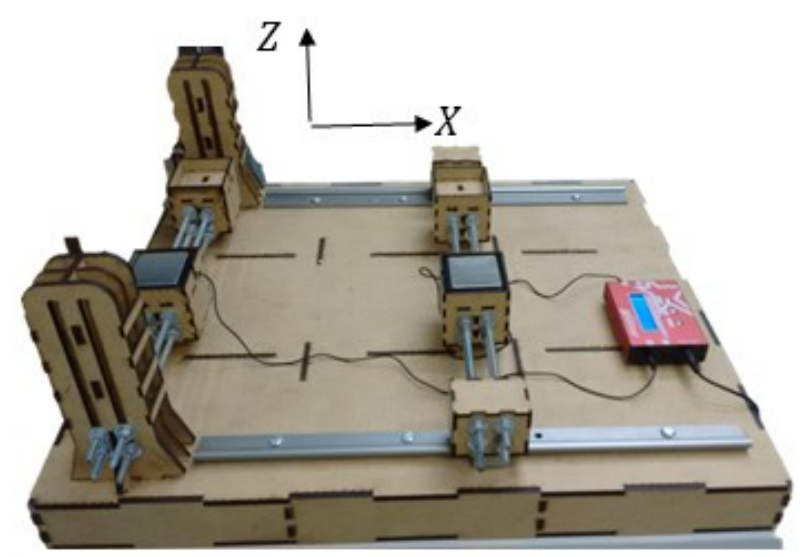

Figure 2.6 - Experimental rig to measure center of mass. 
Table 2.2 - Motorcycle's parameters.

\begin{tabular}{lcl} 
Parameter & Symbol & Value \\
\hline Wheel base & $w$ & $0.311 \mathrm{~m}$ \\
Trail & $t$ & $0.053 \mathrm{~m}$ \\
Caster angle & $\epsilon$ & $0.99 \mathrm{rad}$ \\
Longitudinal speed & $v_{x}$ & Up to $15 \mathrm{~m} / \mathrm{s}$ \\
Gravity & $g$ & $9.81 \mathrm{~m} / \mathrm{s}^{2}$
\end{tabular}

\section{Rear wheel}

\begin{tabular}{|c|c|c|c|}
\hline Radius & $r_{r w}$ & $0.064 \mathrm{~m}$ & \\
\hline Mass & $m_{r w}$ & $0.704 \mathrm{~kg}$ & \\
\hline $\begin{array}{l}\text { Mass moments of } \\
\text { inertia }\end{array}$ & $\left(J_{r w_{x x}} J_{r w_{y y^{\prime}}} J_{r w_{z z}}\right)$ & $\left(\begin{array}{ll}5.30 & 9.39\end{array}\right.$ & $5.30) 10^{-4} \mathrm{~kg}-\mathrm{m}^{2}$ \\
\hline
\end{tabular}

\section{Main body}

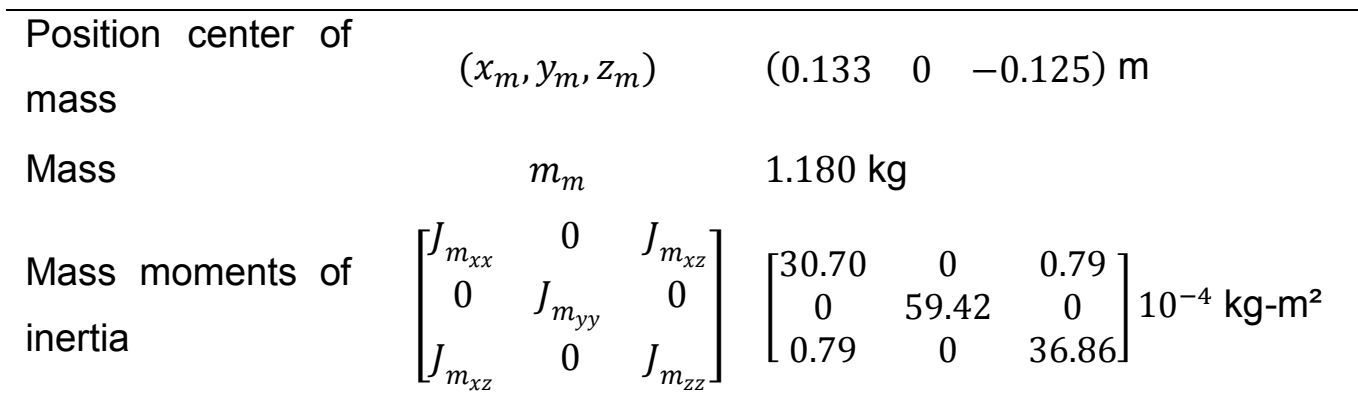

\section{Steering body}

\begin{tabular}{|c|c|c|c|c|}
\hline $\begin{array}{l}\text { Position center of } \\
\text { mass }\end{array}$ & $\left(x_{s}, y_{s}, z_{s}\right)$ & $\left(\begin{array}{ll}0.267 & 0\end{array}\right.$ & $-0.150)$ & $m$ \\
\hline Mass & $m_{s}$ & $0.177 \mathrm{~kg}$ & & \\
\hline $\begin{array}{l}\text { Mass moments of } \\
\text { inertia }\end{array}$ & {$\left[\begin{array}{ccc}J_{s_{x x}} & 0 & J_{s_{x z}} \\
0 & J_{s_{y y}} & 0 \\
J_{s_{x z}} & 0 & J_{s_{z z}}\end{array}\right.$} & {$\left[\begin{array}{cc}6.54 & 0 \\
0 & 7.68 \\
-3.4 & 0\end{array}\right.$} & $\left.\begin{array}{c}-3.4 \\
0 \\
3.19\end{array}\right]$ & $10^{-4} \mathrm{~kg}-\mathrm{m}^{2}$ \\
\hline
\end{tabular}

\section{Front wheel}

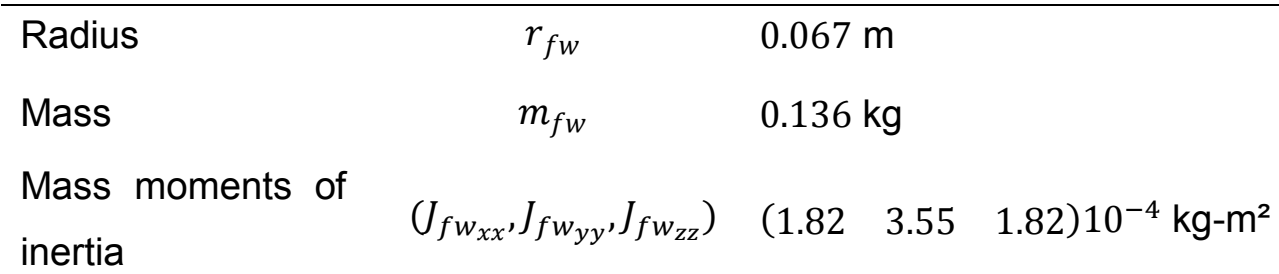


The first test performed was with each scale horizontally aligned under both wheels, as shown in Figure 2.7 and Figure 2.8, to determine the distance between the rear wheel and the center of mass in the $x$ axis. Considering the motorcycle at rest in the described position, the sum of forces and moments acting in the system are given by Eq. (2.1); Table 2.3 brings the test results.

$\left\{\begin{array}{l}\sum F_{z}=m_{t} g=N_{f f}+N_{r f} \\ \sum M_{O}=m_{t} g x_{t}=N_{r f} w\end{array} \therefore x_{t}=\frac{N_{r f} w}{N_{f f}+N_{r f}}\right.$

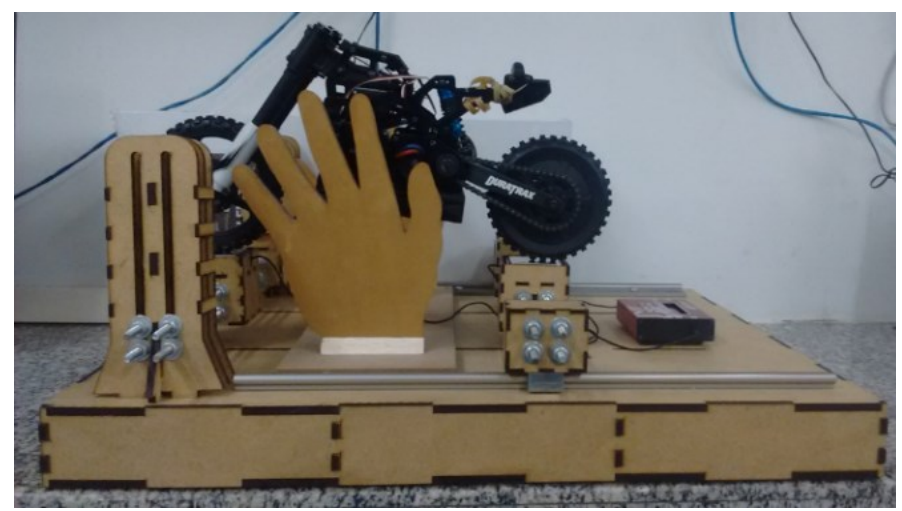

Figure 2.7 - Center of mass measurement in $x$ axis.

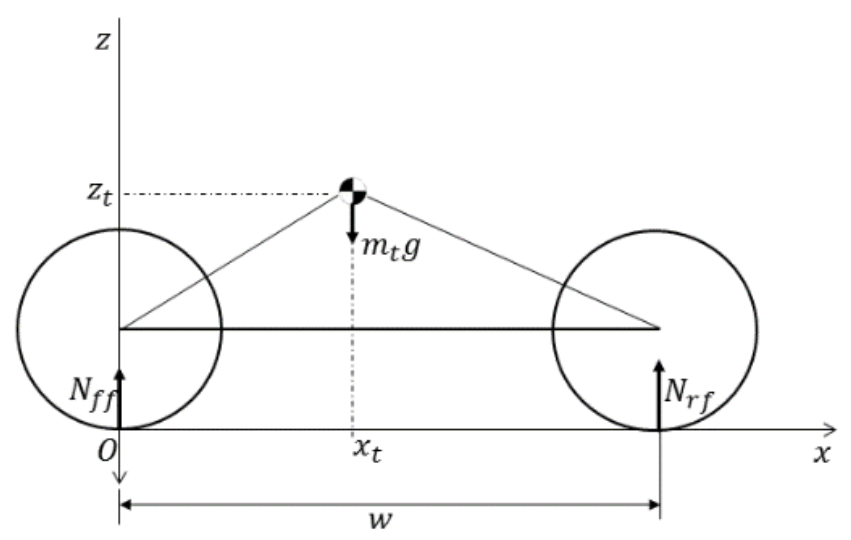

Figure 2.8 - Schematic of center of mass measurement in $x$ axis.

Table 2.3 - Experimental position of $x_{t}$.

\begin{tabular}{|l|l|l|l|l|l|l|}
\cline { 1 - 3 } \multicolumn{1}{|l|}{ Reaction (N) } & \multicolumn{9}{|l|}{} & Mean \\
\cline { 1 - 4 }$N_{r f}$ & 8.432 & 8.514 & 8.395 & 8.380 & 8.357 & 8.416 \\
\hline$N_{f f}$ & 13.675 & 13.636 & 13.724 & 13.685 & 13.734 & 13.691 \\
\hline Mass (kg) & 2.254 & 2.258 & 2.255 & 2.249 & 2.252 & 2.254 \\
\hline $\boldsymbol{x}_{\boldsymbol{t}}(\boldsymbol{m})$ & 0.118 & 0.119 & 0.118 & 0.118 & 0.117 & 0.118 \\
\hline
\end{tabular}


The following test, to determine the center of mass position in the $z$ axis, involved raising the motorcycle at different heights (Figure 2.9 and Figure 2.10) and calculating the center of mass projection at both axis. Eq. (2.2) illustrates the sum of forces and moments acting on the system, while Eq. (2.3) explicit some geometric relations; combining both equations results in the height of the center of mass with respect to the floor-rear wheel contact point (Eq. (2.4)). Table 2.4 to Table 2.6 contains the experimental data for this configuration.

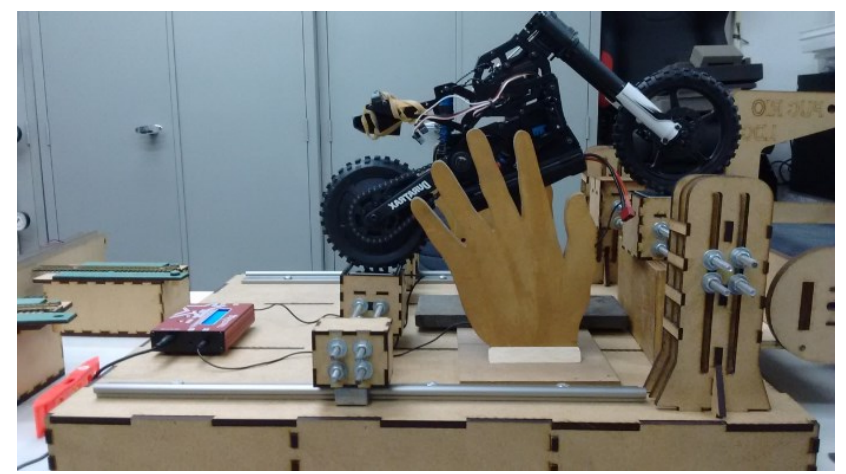

Figure 2.9 - Center of mass measurement in $z$ axis.

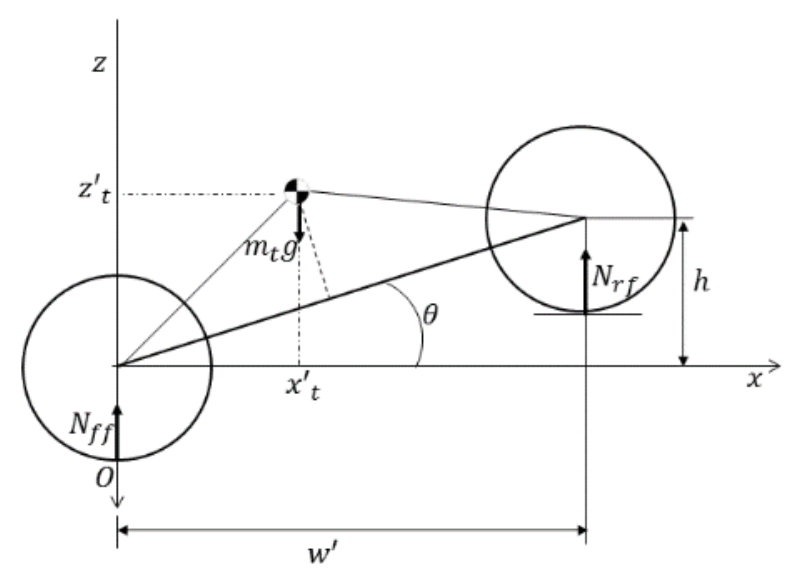

Figure 2.10 - Schematic of center of mass measurement in $z$ axis.

$$
\begin{aligned}
& \left\{\begin{array}{l}
\sum F_{z}=m_{t} g=N_{f f}+N_{r f} \\
\sum M_{O}=m_{t} g x^{\prime}{ }_{t}=N_{r f} w^{\prime}
\end{array} \therefore x^{\prime}{ }_{t}=\frac{N_{r f} w^{\prime}}{N_{f f}+N_{r f}}\right. \\
& \left\{\begin{array}{l}
\sin (\theta)=-h / w \\
x_{t} \cos (\theta)=x_{t}^{\prime}+\left(z_{t}-\left(-r_{r w}\right)\right) \sin (\theta)
\end{array}\right. \\
& z_{t}=-r_{r w}-\frac{x_{t}}{\tan (\theta)}+\frac{N_{r f}}{N_{r f}+N_{f f}} \cdot \frac{w}{\tan (\theta)}=-r_{r w}+\frac{w w^{\prime}}{h}\left[\frac{N_{r f}}{N_{r f}+N_{f f}}-\frac{x_{t}}{w}\right]
\end{aligned}
$$


Table 2.4 - Experimental position of $z_{t}$, with $h=0.135 \mathrm{~m}$.

\begin{tabular}{|l|l|l|l|l|l|l|}
\cline { 1 - 4 } \multicolumn{1}{|l|}{ Reaction (N) } & \multicolumn{5}{|l|}{} & Mean \\
\hline$N_{r f}$ & 7.503 & 7.681 & 7.492 & 7.487 & 7.484 & 7.529 \\
\hline$N_{f f}$ & 14.323 & 14.479 & 14.695 & 14.676 & 14.637 & 14.562 \\
\hline Mass (kg) & 2.225 & 2.259 & 2.262 & 2.259 & 2.255 & 2.252 \\
\hline$z_{\boldsymbol{t}}(\boldsymbol{m})$ & -0.086 & -0.084 & -0.090 & -0.089 & -0.089 & -0.088 \\
\hline
\end{tabular}

Table 2.5 - Experimental position of $z_{t}$, with $h=0.085 \mathrm{~m}$.

\begin{tabular}{|l|l|l|l|l|l|l|}
\cline { 1 - 4 } \multicolumn{1}{|l|}{ Reaction (N) } & \multicolumn{5}{|l|}{} & Mean \\
\hline$N_{r f}$ & 8.020 & 8.083 & 8.113 & 8.319 & 8.093 & 8.126 \\
\hline$N_{f f}$ & 14.784 & 14.951 & 14.833 & 14.951 & 14.892 & 14.882 \\
\hline Mass (kg) & 2.325 & 2.348 & 2.339 & 2.372 & 2.343 & 2.345 \\
\hline$z_{\boldsymbol{t}}(\boldsymbol{m})$ & -0.093 & -0.094 & -0.091 & -0.087 & -0.093 & -0.091 \\
\hline
\end{tabular}

Table 2.6 - Experimental position of $z_{t}$, with $h=0.045 \mathrm{~m}$.

\begin{tabular}{|l|l|l|l|l|l|l|}
\cline { 1 - 4 } Reaction (N) & \multicolumn{5}{|l|}{} & Mean \\
\hline$N_{r f}$ & 8.196 & 8.240 & 8.281 & 8.341 & 8.184 & 8.248 \\
\hline$N_{f f}$ & 14.019 & 13.950 & 13.921 & 13.852 & 13.969 & 13.942 \\
\hline Mass (kg) & 2.265 & 2.262 & 2.263 & 2.262 & 2.258 & 2.262 \\
\hline$z_{\boldsymbol{t}}(\boldsymbol{m})$ & -0.086 & -0.081 & -0.080 & -0.072 & -0.085 & -0.081 \\
\hline
\end{tabular}

The final results and comparison between the center of mass calculated via software and experimentally are summarized in Table 2.7. The errors between theoretical and experimental values are approximately 6 and 1 milimeter for the $x$ and $z$ axis. Due to the small errors between experimental and software results, it is assumed the other simulated variables are representative of the real system, such as moments of inertia and mass distribution.

Table 2.7 - Center of mass via software and experiments.

\begin{tabular}{|l|l|l|}
\hline Axis & Software (m) & Experimental (m) \\
\hline$x_{t}$ & 0.112 & 0.118 \\
\hline$z_{t}$ & -0.089 & -0.088 \\
\hline
\end{tabular}




\subsection{Traction system}

The traction system is actuated by an electric brushless DC motor, a three-phase synchronous motor powered by a constant voltage source, passing through an integrated inverter that will produce the alternating electrical signal needed to start the motor. This type of engine is usually controlled by an electronic circuit known as ESC (electronic speed control); capable of varying the speed and direction of rotation of the electric motor, in addition to being able to function as a dynamic brake. The original radio signal to the ESC was substituted by a pulse-width modulation signal $(P W M)$ sent by the main microcontroller; additionally, the reverse and brake functions were disabled, meaning the motor has angular speed only in one direction (forward). Table 2.8 brings the main technical features of the brushless motor.

Table 2.8 - Traction motor technical features.

\begin{tabular}{|l|l|}
\hline Manufacturer & DURATRAX/ DTXC4602 \\
\hline Motor type & Brushless DC \\
\hline Signal & Pulse-Width Modulation (PWM) \\
\hline Velocity constant & $3900[$ RPM/V] \\
\hline Battery type & $7,2 \mathrm{~V} \mathrm{NiCd/NiMH} \mathrm{ou} \mathrm{7,4V} \mathrm{LiPo}$ \\
\hline
\end{tabular}

To characterize the traction system, two experiments were performed: the first was to identify the torque applied to the system, detailed in Appendix B; the information is useful for a velocity control strategy, which was not on the subject of this work. The second experiment was to correlate the command signal and the motorcycle's final longitudinal speed; considering no slippage on neither tires, the rear wheel angular speed has a direct relation with the linear velocity. The test used a tachometer connected to the brushless motor and a Hall effect sensor attached with four magnets equally distributed onto the rear wheel.

The transmission system, illustrated on Figure 2.11, is defined by four gears: the first $\left(E_{1}\right)$ is directly connected to the motor engine and the last $\left(E_{4}\right)$, to the rear wheel. The sizes of each gear is detailed on Table 2.9; the ratio between the motor and rear wheel angular speeds $(N)$ is defined by Eq. (2.5), which results in 14.45 to the specified system - this means the motor rotates almost fifteen times faster than the rear wheel. 
Table 2.9 - Transmission system.

\begin{tabular}{|l|l|l|l|l|}
\hline Gear & E1 & E2 & E3 & E4 \\
\hline Number of teeth & 13 & 65 & 9 & 26 \\
\hline
\end{tabular}

$\{\begin{array}{lll}v_{E 1}=v_{E 2} & \omega_{E 1} N_{E 1}=\omega_{E 2} N_{E 2} \\ \omega_{E 2}=\omega_{E 3} & \therefore & \omega_{E 2}=\omega_{E 3} \\ v_{E 3}=v_{E 4} & \omega_{E 3} N_{E 3}=\omega_{E 4} N_{E 4}\end{array} \Rightarrow \omega_{E 1}=\underbrace{\frac{N_{E 2} N_{E 4}}{N_{E 1} N_{E 3}}}_{N} \omega_{E 4}$
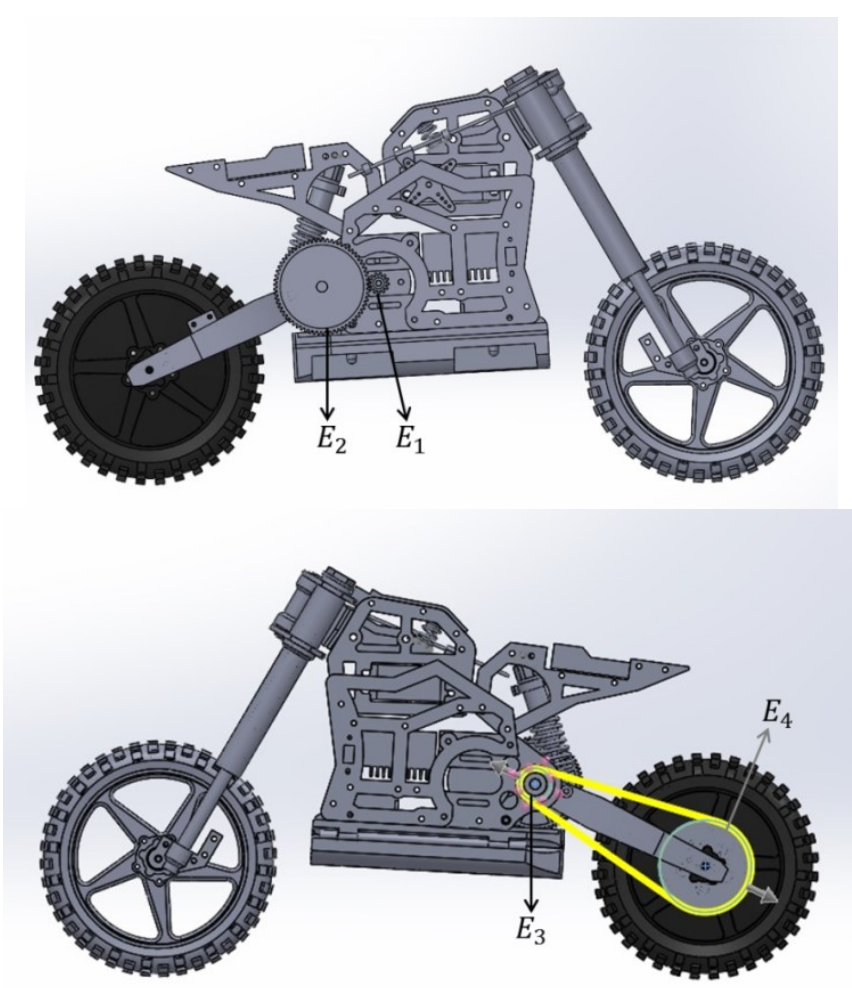

Figure 2.11 - Transmission system computational model.

The tests consisted of varying the input signal sent to the brushless motor coupled directly to the motorcycle's rear wheel, which could spin freely without contact with the ground. The experiment consisted of an increasing and decreasing command signal with step amplitude of $10 \%, 20 \%, 30 \%, 40 \%$ and $50 \%$, ignoring the range between $70 \%$ and $100 \%$ - where the motor presents a saturation and no increase of angular speed is observed.

Due to the transmission system, the maximum amplitude variation is $50 \%$ between input signals before an overcurrent, when the motor is already spinning; from the start, all tests were first stabilized at $10 \%$. The results of the motor and rear wheel angular speeds are in Figure 2.12 to Figure 2.13; the experimental ratio between both velocities is, in average, 14.52 , very close to the result calculated via the transmission setup. 
Considering the angular speeds obtained in this experiment as an upper limit to the velocity developed by the motorcycle in contact with the floor, it is possible to calculate the maximum longitudinal speed of the vehicle through a linear relation (Eq. (2.6)). Since the highest angular speed for the wheel is approximately $240 \mathrm{rad} / \mathrm{s}$ and the rear wheel radius is in Table 2.2, the maximum linear speed for the studied vehicle is $14.5 \mathrm{~m} / \mathrm{s}$.

$v_{x}=\omega_{\text {wheel }} \times r_{r w}$
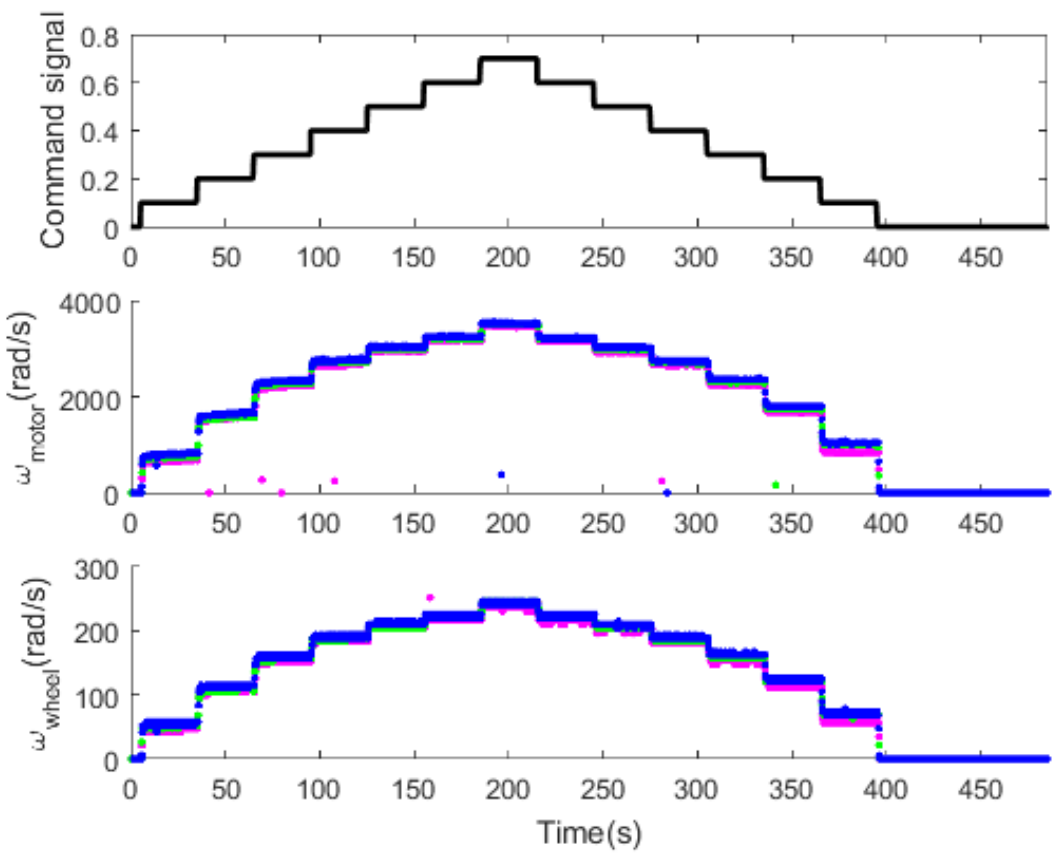

Figure 2.12 - Transmission system test with $10 \%$ step.
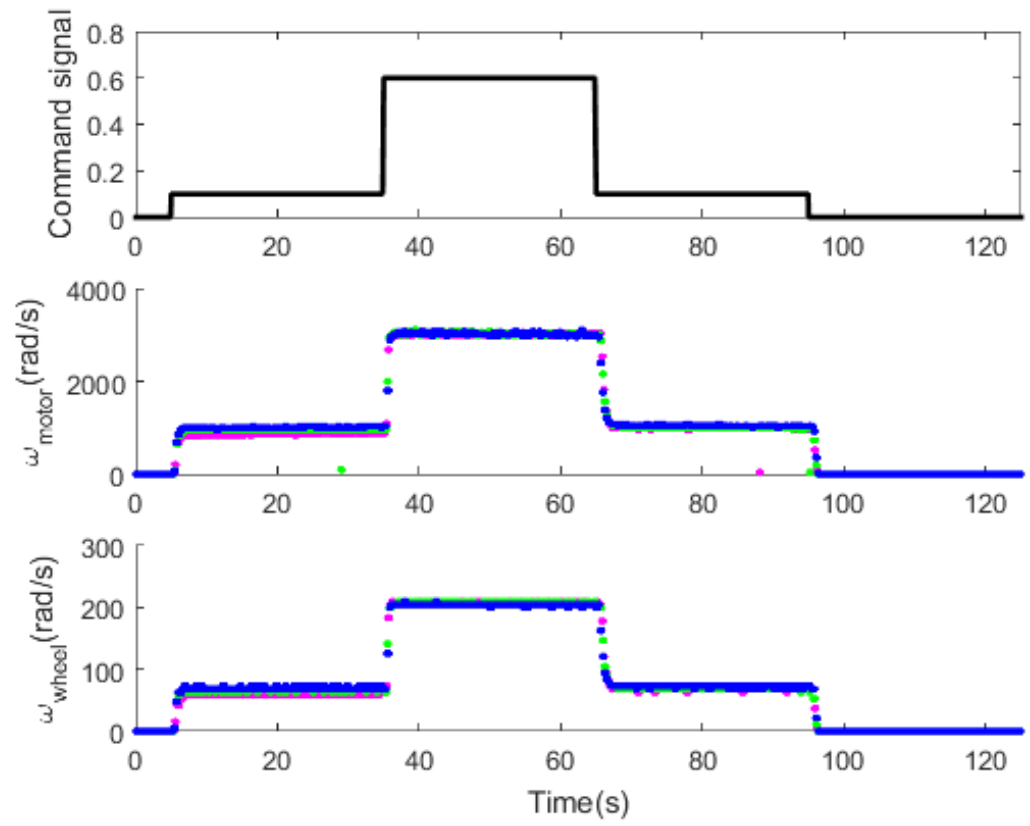

Figure 2.13 - Transmission system with $50 \%$ step. 


\subsection{Steering system}

The steering system is actuated by a servomotor - an electric motor with feedback, capable of position (angle) control. The device is also controlled by a Pulse Width Modulation (PWM) signal sent by the microcontroller, where each different duty cycle represents a desired angle position, varying from 0 to 60 degrees. Further information of the servo used is at Table 2.10.

Table 2.10 - Steering motor technical features.

\begin{tabular}{|l|l|}
\hline Manufacturer & TACTIC/ TSX200 \\
\hline Motor type & Servomotor \\
\hline Signal & Pulse Width Modulation (PWM) \\
\hline Voltage & $4.8 \mathrm{~V}$ \\
\hline Torque & $0.32 \mathrm{~N} . \mathrm{m}$ \\
\hline Speed (without load) & $0.16 \mathrm{sec} / 60$ degrees or $6.54 \mathrm{rad} / \mathrm{s}$ \\
\hline
\end{tabular}

The next step to characterize the steering system is to correlate the signal sent by the microcontroller and the final angle made by the steering axis; the experiment setup is illustrated on Figure 2.14. Using a protractor, the angle between the front wheel and the main body/rear wheel axis is measured; a potentiometer, coupled to the motorcycle handlebar, yields the same information with an electric voltage variation, read by the Arduino and sent to the Pixhawk via a serial communication. Following the right-hand rule and the proposed axis configuration, clockwise rotations on the handlebar are considered positive, i.e., steer angles to the right are positive and to the left, negative.

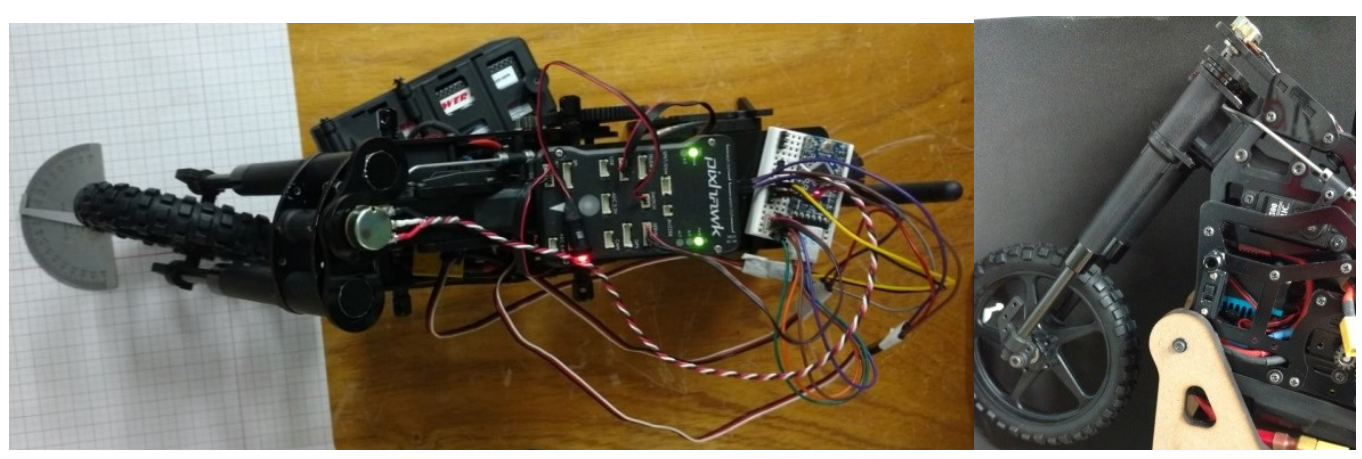

Figure 2.14 - Steering system experiment setup. 
The final relation between the potentiometer readings and the measured steering angle - in radians - is given by Eq. (2.7) and illustrated in Figure 2.15. The potentiometer signal is interpreted as an analog input by the Arduino, with range of 1024; after the normalization, the signal has a bias of 0.49 , removed at further analysis. The proposed linear fit presents an error of $5.65 \%$.

$\delta=-3.91$ Pot +0.061

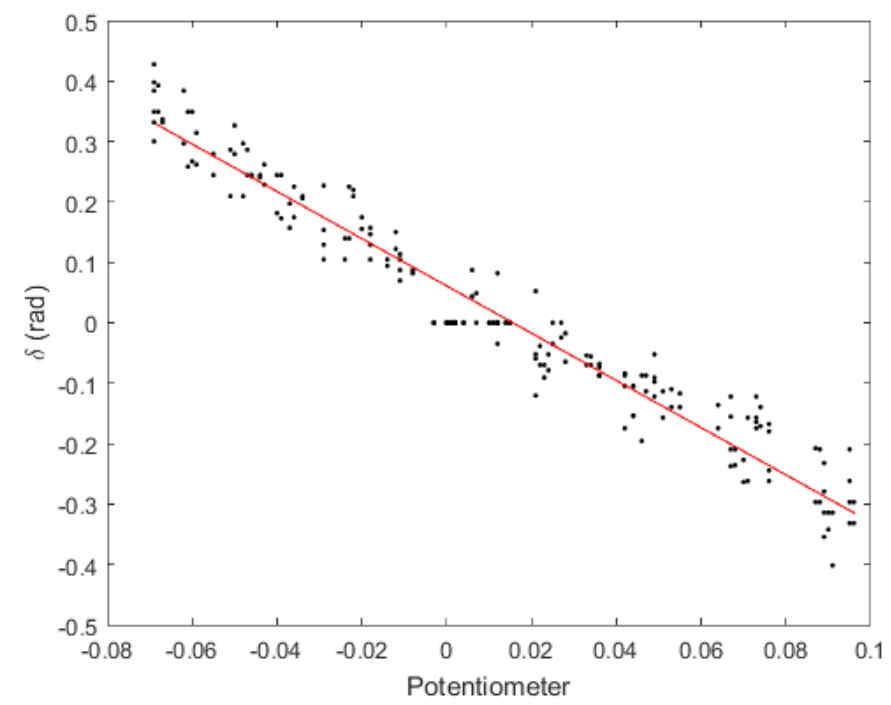

Figure 2.15 - Potentiometer versus steering angle.

Afterwards, it is necessary to stablish the relation between the command signal sent by the microcontroller and the resultant front wheel steer angle. Respecting the physical assembly and constraints of the system, the command signals sent to the servomotor vary from zero to -0.7 ; the front wheel is aligned with the main body at a -0.3 input, which indicates that the handlebar steers further to the left than to the right.

Removing the servomotor and potentiometer's bias and using Eq. (2.7), it is possible to correlate the command signal to the servo and final front wheel steer angle, in radians. Figure 2.17 and Figure 2.18 bring the experimental result to different step amplitudes between commands, at distinct start points; the angle response to the servo actuation is almost instantaneous, unlike the traction system, in which the inertial load takes up to three seconds to achieve steady state. Therefore, a linear relation between steering angle and command signal can be extrapolated from the data (Eq. (2.8) and Figure 2.16), with 1.23\% 
of error. The previous images also illustrate the proposed linear fit, with a maximum error of 0.07 rad, or approximately 5 degrees, representing $10 \%$ of the total cursor.

$\delta=0.86 u_{s}+0.07$

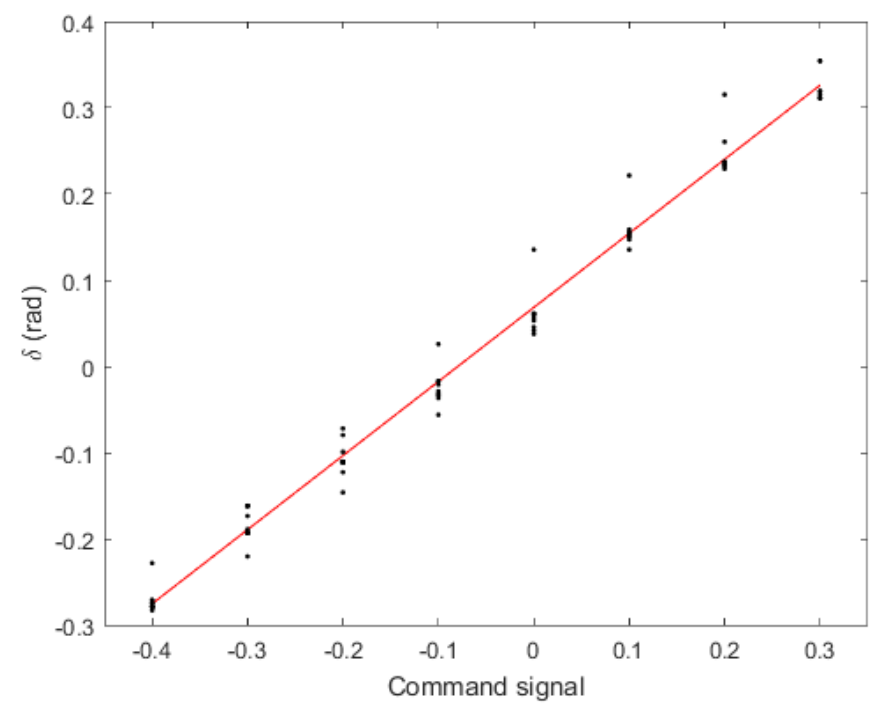

Figure 2.16 - Command signal versus steering angle.

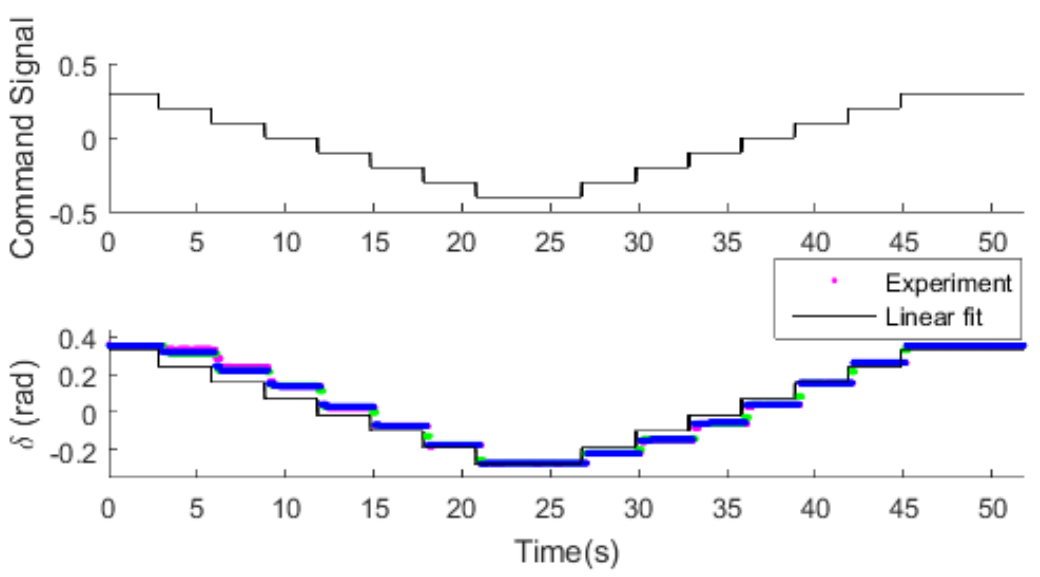

Figure 2.17 - Steering system with $10 \%$ step. 


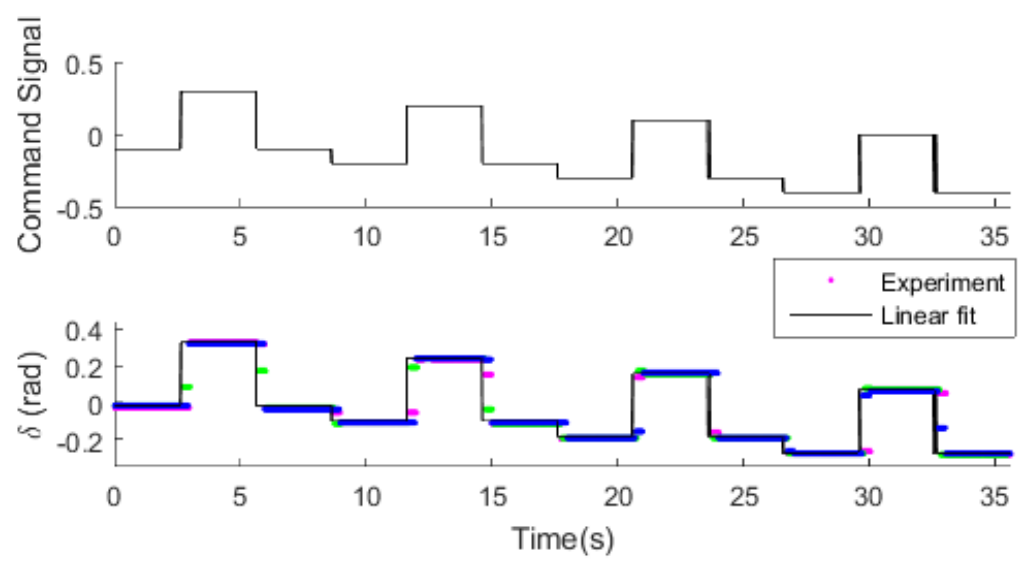

Figure 2.18 - Steering system with $40 \%$ step.

This chapter presented the chosen experimental vehicle for this work: a scale electric motorcycle, in which the proposed control algorithms will be implemented. The study object had its main components characterized. First, the data flow was detailed: the main microcontroller receives the instrumentation measurements, executes the control algorithm and sends the resulting commands to both electric motors. Then, its geometric and inertial parameters were identified via computational simulation and ratified through simple experiments.

At last, both electric motors were characterized and its coupled sensors were calibrated: a relation was determined between respective command signals and the final steady state obtained (longitudinal speed for the traction system and steering angle for the steering system).

In the next chapter, a two-wheeled vehicle dynamic model is presented, in which the parameters determined in the experimental characterization of the system are used. 


\section{Two-wheeled vehicle dynamics}

In the previous chapter, the experimental scale motorcycle was characterized, with its principal parameters being determined through computational simulations and simple test rigs. This chapter presents a linear dynamic model for two-wheeled vehicles (Sharp, 1971 and Meijaard, 2007) based on cited parameters and analyzes the self-stability of the uncontrolled scale motorcycle.

A detailed and realistic motorcycle's dynamic model is highly complex due to the vehicle's many degrees of freedom and non-trivial geometry; in order to simplify the model while keeping its dynamic effects, some aspects have to be taken into consideration such as which components can be omitted, how to deal with the wheel-to-ground interaction and the complexity that should be given to the driver model.

In this work, the motorcycle is considered as a four rigid bodies system: two wheels, the main body and the steering body, consisting of a front fork with handlebar; the influence of other usual parts of the vehicle - belts, shock absorber, pedals, brake system - are neglected. Tires deformity and slippage are not considered, i.e., the wheels provide exclusively rolling, non-holonomic constraints on the longitudinal, lateral and yawing motions of the vehicle. The longitudinal speed of the vehicle is considered constant and the steering system is free to steer itself.

Since the final objective of this work is to build an autonomous vehicle, the driver - or controller system - is represented in the dynamic model solely by the torque applied to the handlebars, without taking into account its mass on the main body or the double inverted pendulum phenomenon that physically exists with a real pilot.

Next, the procedure to obtain the motorcycle dynamic model is detailed, based on the Whipple bicycle model, and taking into account the two main elements of interest to the autonomous two-wheeled system: stability and trajectory control. 


\subsection{Linearized dynamic model}

The linear model was built based on Sharp's bicycle model (1971), where the multibody approach to a two-wheeled vehicle considers two structures, or frames, joined together by a revolution joint in the handlebars, with each frame holding a free to rotate axisymmetric wheel. Both frames are considered having a uniformly distributed mass and the wheels provide purely a rotational movement, i.e., there is no tire deformation or slippage.

In the original work, the driver is modelled as a rigid extension to the main frame, which results in a higher and closer to the rear center of mass, since the average human body is heavier than the usual bicycle frame. This center mass final location is particularly true in motorcycles, with a heavier frame, so the same hypothesis can be applied on this system, even on an unmanned vehicle.

In order to reduce the model complexity, the four body have been simplified to two sets: the front frame includes the handlebar, fork and front wheel; the rear frame consists of the main body and rear wheel. Figure 3.1 illustrates the model's main parameters, based on Meijjard (2007): the roll, pitch and yaw angles are defined as, respectively, $\varphi, \theta, \psi$, the steering angle is $\delta$; some geometric parameters such as the wheel base $(w)$, caster angle or head trail $(\epsilon)$ and trail $(t)$ are also depicted, alongside with the approximate front and rear frames center of mass localization $\left(m_{f f}, m_{r f}\right)$.

Figure 3.2 brings the global and local coordinates systems, the latter with origin at the rear wheel and floor contact point, indicated as $O$ in the figures. It is worth emphasizing the unusual coordinate orientation, with $z$ axis being positive in gravity's favor; this configuration allows for a positive angular speed when the vehicle is falling or heading to the right, i.e., the roll and yaw rates are positive when clockwise. The same principle applies to the steering angle, with right steer as positive, but the wheel rotation has a negative forward motion.

The motorcycle and bicycle multibody model is defined by 25 design parameters, which are further detailed on Appendix A. The experimental numerical parameters of the small scale vehicle analyzed in this work also appear on Table 2.2. With these parameters and considering a two-wheeled vehicle moving freely in a plane with constant longitudinal speed $\left(v_{x}\right)$, the linearized motion equations of the system are based on two main degrees of freedom: the roll and steer angles ( $\varphi$ and $\delta)$. 


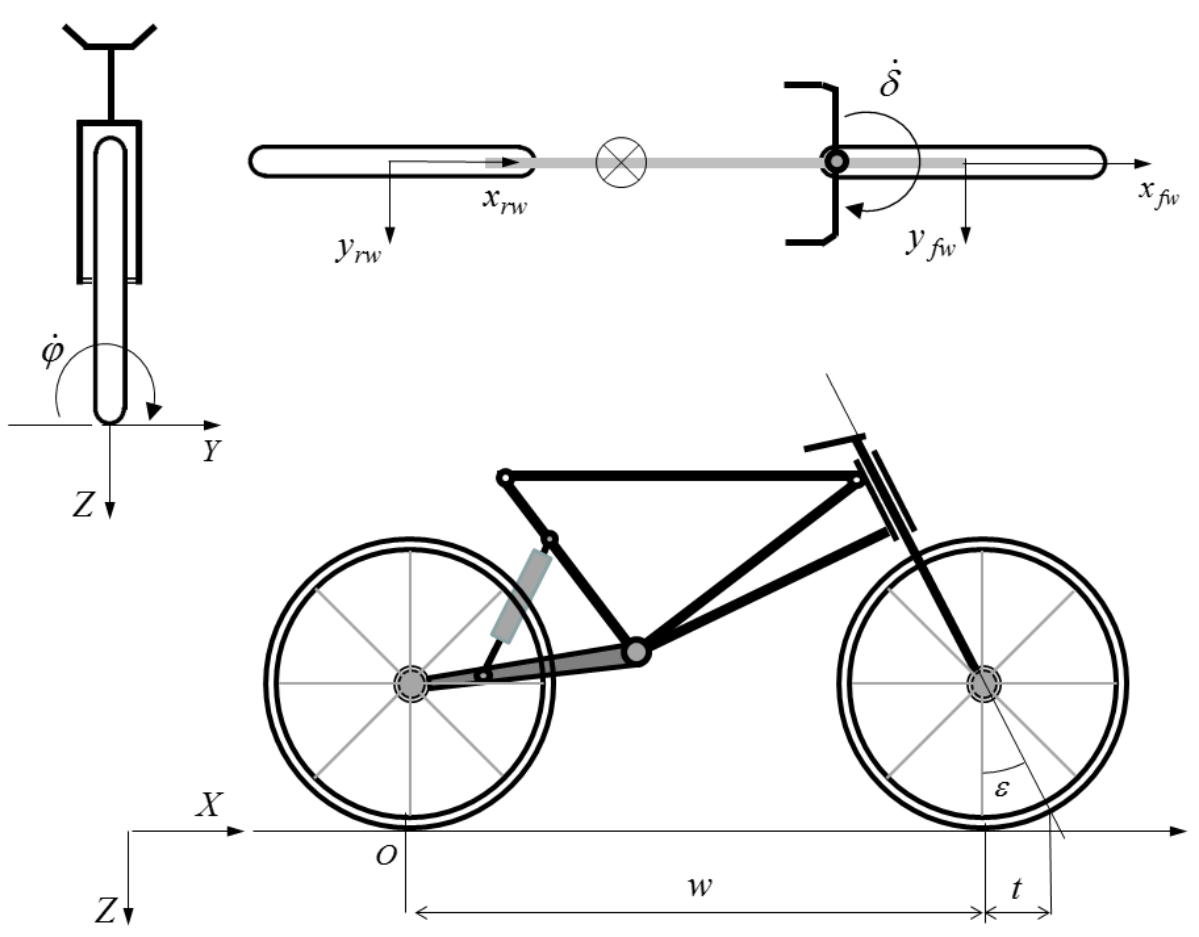

Figure 3.1 - Motorcycle geometry main parameters.

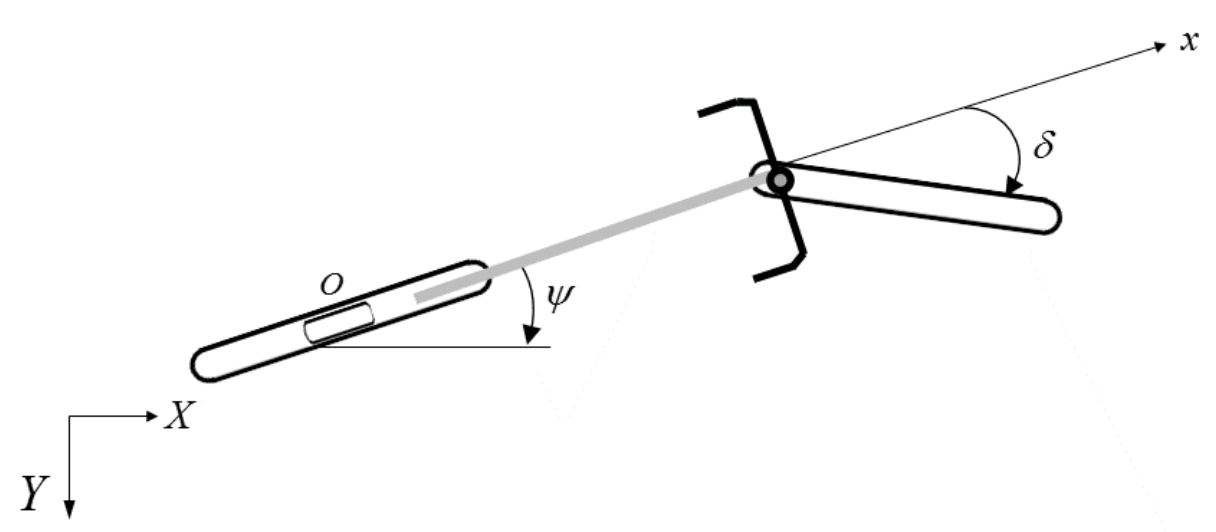

Figure 3.2 - Global and local coordinate systems.

Other important parameters to the linear model are the gravity at each mass center $\left(m_{f f}, m_{r f}\right)$, positive in the $z$ direction; vertical and horizontal ground reaction force at the front wheel $\left(-g m_{t} x_{t} / w\right.$ and $F_{y}$, respectively); a lean moment $\left(T_{\varphi}\right)$ applied to the rear frame by the driver, responsible for the motorcycle rolling; a steer torque $\left(T_{\delta}\right)$ applied to the handlebar, positive when clockwise, and applied in the opposite direction in the rear frame.

The three main dynamic equations are derived from the angular momentum balance for the roll, steer and yaw $(\psi)$ movements, respectively Eq. (3.1) to (3.3). The only unknown force, the front wheel lateral reaction $F_{y}$, can be eliminated by combining Equations (3.2) and (3.3). 


$$
\begin{gathered}
-m_{t} z_{t} \ddot{y}_{r w}+J_{x x} \ddot{\varphi}+J_{x z} \ddot{\psi}+J_{\epsilon x} \ddot{\delta}+v_{x} S_{t} \dot{\psi}+v_{x} S_{f} \cos (\epsilon) \dot{\delta}= \\
=T_{\varphi}-g m_{t} z_{t} \operatorname{sen}(\varphi)+g S_{u} \operatorname{sen}(\delta) \\
m_{f f} u \ddot{y}_{r w}+J_{\epsilon x} \ddot{\varphi}+J_{\epsilon z} \ddot{\psi}+J_{\epsilon \epsilon} \ddot{\delta}+v_{x} S_{f}(-\dot{\varphi} \cos (\epsilon)+\dot{\psi} \operatorname{sen}(\epsilon))= \\
=T_{\delta}-t \cos (\epsilon) F_{y}+g S_{u}(\operatorname{sen}(\varphi)+\operatorname{sen}(\delta) \operatorname{sen}(\epsilon)) \\
m_{t} x_{t} \ddot{y}_{r w}+J_{x z} \ddot{\varphi}+J_{z z} \ddot{\psi}+J_{\epsilon z} \ddot{\delta}-v_{x} S_{t} \dot{\varphi}-v_{x} S_{f} \operatorname{sen}(\epsilon) \dot{\delta}-w F_{y}=0
\end{gathered}
$$

Assuming small roll, steer and yaw angles, the motorcycle would be moving almost parallel to the global $x$-axis, so the wheels forward speeds, $\dot{y}_{f w}$ and $\dot{y}_{r w}$, can be simplified to Eq. (3.4) and (3.5), obtained via kinematic relations. The last result, Eq. (3.6), is an equation correlating the yaw and steer angles whose time derivative can be substituted in the previous equations.

$$
\begin{aligned}
& \dot{y}_{r w}=v_{x} \psi \\
& \begin{aligned}
\dot{y}_{f w} & =\frac{d}{d t}\left(y_{r w}+w \psi-t \delta \cos (\epsilon)\right)=v_{x} \psi+v_{x} \delta \cos (\epsilon) \\
& =\dot{y}_{r w}+w \dot{\psi}-t \dot{\delta} \cos (\epsilon)=v_{x} \psi+v_{x} \delta \cos (\epsilon)
\end{aligned} \\
& \dot{\psi}=\frac{\cos (\epsilon)}{w}\left[t \dot{\delta}+v_{x} \delta\right]
\end{aligned}
$$

Therefore, eliminating the variable $F_{y}$ and considering the previous rolling constraints, the three angular momentum balance equations become two linear equations with just the roll and steer (and their time derivative) angles as the unknown variables, as explicit in Eq. (3.7) and (3.8).

$$
\begin{gathered}
J_{x x} \ddot{\varphi}+g m_{t} z_{t} \varphi+\left[J_{\epsilon x}+f J_{x z}\right] \ddot{\delta}+\left[S_{f} \cos (\epsilon)+f\left(S_{t}-m_{t} z_{t}\right)+\right. \\
\left.+\frac{\cos (\epsilon)}{w} J_{x z}\right] v_{x} \dot{\delta}+\left[\left(S_{t}-m_{t} z_{t}\right) \frac{\cos (\epsilon)}{w} v_{x}^{2}-g S_{u}\right] \delta=T_{\varphi} \\
{\left[f J_{x z}+J_{\epsilon x}\right] \ddot{\varphi}-\left[f S_{t}+S_{f} \cos (\epsilon)\right] v_{x} \dot{\varphi}-g S_{u} \varphi+\left[f^{2} J_{z z}+2 f J_{\epsilon z}+J_{\epsilon \epsilon}\right] \ddot{\delta}} \\
+ \\
+\left[f S_{u}+\frac{\cos (\epsilon)}{w}\left(f J_{z z}+J_{\epsilon z}\right)\right] v_{x} \dot{\delta}+\left[\left(S_{u}+S_{f} \operatorname{sen}(\epsilon)\right) \frac{\cos (\epsilon)}{w} v_{x}^{2}-\right. \\
\left.-g S_{u} \operatorname{sen}(\epsilon)\right] \delta=T_{\delta}
\end{gathered}
$$


Finally, the system can be written in matrix form, which gives Eq. (3.9), summarized in Eq. (3.10) - with $q=\left[\begin{array}{ll}\varphi & \delta\end{array}\right]^{T}$ and $\tau=\left[\begin{array}{ll}T_{\varphi} & T_{\delta}\end{array}\right]^{T}$.

$$
\begin{aligned}
& \left.\begin{array}{cc}
J_{x x} & f J_{x z}+J_{\epsilon x} \\
f J_{x z}+J_{\epsilon x} & f^{2} J_{z z}+2 f J_{\epsilon z}+J_{\epsilon \epsilon}
\end{array}\right]\left[\begin{array}{l}
\ddot{\varphi} \\
\ddot{\delta}
\end{array}\right]+ \\
& +v_{x}\left[\begin{array}{cc}
0 & S_{f} \cos (\epsilon)+f\left(S_{t}-m_{t} z_{t}\right)+J_{x z} \cos (\epsilon) / w \\
-f S_{t}-S_{f} \cos (\epsilon) & f S_{u}+\left(f J_{z z}+J_{\epsilon z}\right) \cos (\epsilon) / w
\end{array}\right]\left[\begin{array}{l}
\dot{\varphi} \\
\dot{\delta}
\end{array}\right] \\
& +\quad\left(g\left[\begin{array}{cc}
m_{t} z_{t} & -S_{u} \\
-S_{u} & -S_{u} \operatorname{sen}(\epsilon)
\end{array}\right]+v_{x}^{2}\left[\begin{array}{ll}
0 & \left(S_{t}-m_{t} z_{t}\right) \cos (\epsilon) / w \\
0 & \left(S_{u}+S_{f} \operatorname{sen}(\epsilon)\right) \cos (\epsilon) / w
\end{array}\right]\right)\left[\begin{array}{l}
\varphi \\
\delta
\end{array}\right] \\
& =\left[\begin{array}{l}
T_{\varphi} \\
T_{\delta}
\end{array}\right] \\
& M \ddot{q}+v_{x} C \dot{q}+\left(g K_{0}+v_{x}^{2} K_{2}\right) q=\tau
\end{aligned}
$$

Eq. (3.10) allows for a parallel with the mechanical mass-spring-damper second order model. $M$ is a symmetric and positive-defined matrix and corresponds to the mass matrix. The matrix $C$ functions as the damping system, linearly dependent to the longitudinal speed, and illustrates the gyroscopic torques due to steer and roll rates as well as inertial reactions due to steer rate. At last, $K$ is the stiffness matrix, formed by two subsystems: a part proportional to the gravity $\left(K_{0}\right)$, which gives potential energy information, and a part quadratic in the forward speed $\left(K_{2}\right)$, which gives the gyroscopic and centrifugal effects. The system can also be represented in a state space form, which gives Eq. (3.11).

$$
\left[\begin{array}{c}
\ddot{q} \\
\dot{q}
\end{array}\right]=\left[\begin{array}{cc}
-M^{-1} v_{x} C & -M^{-1}\left(g K_{0}+v_{x}^{2} K_{2}\right) \\
I_{2 \times 2} & 0_{2 \times 2}
\end{array}\right]\left[\begin{array}{l}
\dot{q} \\
q
\end{array}\right]+\left[\begin{array}{c}
M^{-1} \\
0_{2 \times 2}
\end{array}\right][\tau]
$$

Using the numerical results from Table 2.2 and the parameters described on Appendix A, the state space model is explicit on Eq. (3.12). 


$$
\begin{gathered}
\dot{X}=A X+B U \\
{\left[\begin{array}{c}
\ddot{\varphi} \\
\ddot{\delta} \\
\dot{\varphi} \\
\dot{\delta}
\end{array}\right]=\left[\begin{array}{cccc}
-0.48 v_{x} & -1.9 v_{x} & 75 & -14 v_{x}^{2}-13 \\
2.7 v_{x} & -3.5 v_{x} & -6.6 & 0.33 v_{x}^{2}+144 \\
1 & 0 & 0 & 0 \\
0 & 1 & 0 & 0
\end{array}\right]\left[\begin{array}{c}
\dot{\varphi} \\
\dot{\delta} \\
\varphi \\
\delta
\end{array}\right]} \\
+\left[\begin{array}{cc}
51 & -100 \\
-100 & 566 \\
0 & 0 \\
0 & 0
\end{array}\right]\left[\begin{array}{l}
T_{\varphi} \\
T_{\delta}
\end{array}\right]
\end{gathered}
$$

At last, the model is missing only the global position of the vehicle in the inertial reference axis to fully represent its state. The yaw angle, described on Eq. (3.6), is dependent of the motorcycle longitudinal speed and the steering state variables - both angular speed and position. Considering that the vehicle does not slip, the rear contact point position, therefore, can be described by Equation (3.13).

$$
\dot{x}_{r w}=v_{x} \cos (\psi) \quad \dot{y}_{r w}=v_{x} \sin (\psi)
$$

With the small angle hypothesis, the previous equation can be linearized: the motorcycle global position on the $x$ axis is the integration of the constant longitudinal speed and the $y$ axis has a linear relation between its acceleration and the yaw angular speed, given by Eq. (3.14).

$\ddot{y}_{r w}=v_{x} \dot{\psi}=\frac{\cos (\epsilon)}{w}\left[v_{x} t \dot{\delta}+v_{x}^{2} \delta\right]$

Finally, Eq. (3.6) and (3.14) are added to the output of the state-space model, described on Eq. (3.15), already with the numerical values from Table 2.2 .

$Y=C X+D U$

$\left[\begin{array}{c}\dot{\varphi} \\ \dot{\delta} \\ \varphi \\ \delta \\ \dot{\psi} \\ \ddot{y}_{r w}\end{array}\right]=\left[\begin{array}{cccc}1 & 0 & 0 & 0 \\ 0 & 1 & 0 & 0 \\ 0 & 0 & 1 & 0 \\ 0 & 0 & 0 & 1 \\ 0 & 0.09 & 0 & 1.76 v_{x} \\ 0 & 0.09 v_{x} & 0 & 1.76 v_{x}^{2}\end{array}\right]\left[\begin{array}{c}\dot{\varphi} \\ \dot{\delta} \\ \varphi \\ \delta\end{array}\right]+0_{6 \times 2}\left[\begin{array}{c}T_{\varphi} \\ T_{\delta}\end{array}\right]$ 


\subsection{Analysis of the uncontrolled vehicle}

In the previous section, the mathematical model of a two-wheeled vehicle, controlled by torques in the handlebar (steering) and rear frame (rolling), was developed. However, it is known that, for certain speed ranges, bicycles and motorcycles are able to remain stable without the driver aid. Therefore, it is essential to analyze the stability of the uncontrolled motorcycle, with zero input torque.

The motorcycle lateral movements stability depends on the four poles to the system's characteristic equation, that is, the eigenvalues to matrix $A$ defined on Eq. (3.12). Considering the geometry of typical motorcycles and bicycles, the fourth order model has usually two real and one pair of complex eigenvalues.

The smallest real eigenvalue - with positive or negative part - is associated with capsize mode, depicted in Figure 3.3, related to the roll angle. This mode happens when the vehicle falls sideways with the rolling angle increasing slowly, in a tightening spiral movement.
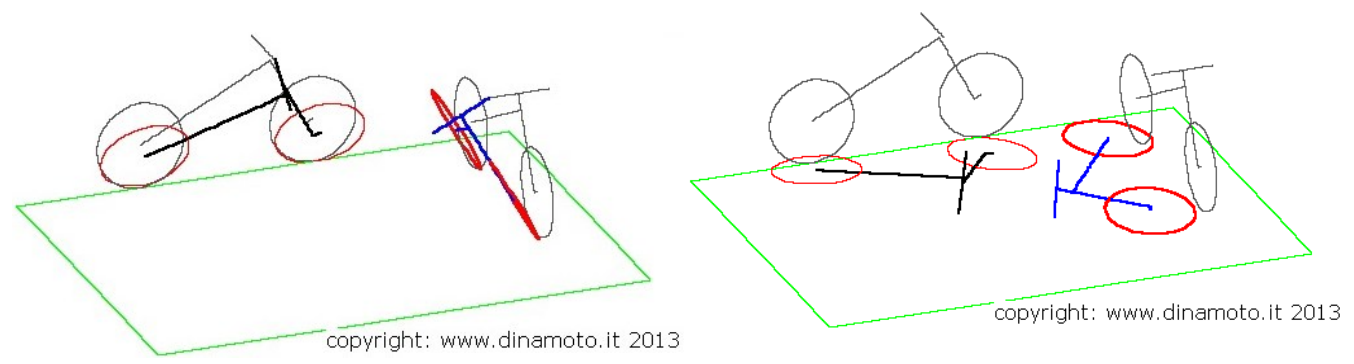

Figure 3.3 - Capsize mode, by <www.dynamotion.it>.

The second non-oscillating mode is the castering, associated with the larger real eigenvalue and the steer angle. This characteristic movement occurs when the front frame rapidly aligns with the headed direction, following the steering motion. This mode is typically associated with a negative real pole and, therefore, represents a stable behavior. This phenomenon happens because the extension of the steering axis is slightly in front of the front wheel-floor contact point (the trail distance), which allows this 'following the path' pattern, like the caster wheels in shopping carts or furniture. 
The weave mode is an oscillatory movement (Figure 3.4), where the motorcycle oscillates around the headed direction, usually with a slight phase lag between the steer and roll angles, resulting in a sinuously path. This mode is associated with the pair of conjugated complex eigenvalues and since its real part can be either positive or negative, the final movement may result in, respectively, an unstable or stable trajectory. At low speeds, the unstable mode may be easily corrected but, at high speeds, its natural frequency may be too high to be controlled by the driver, which could be potentially dangerous.
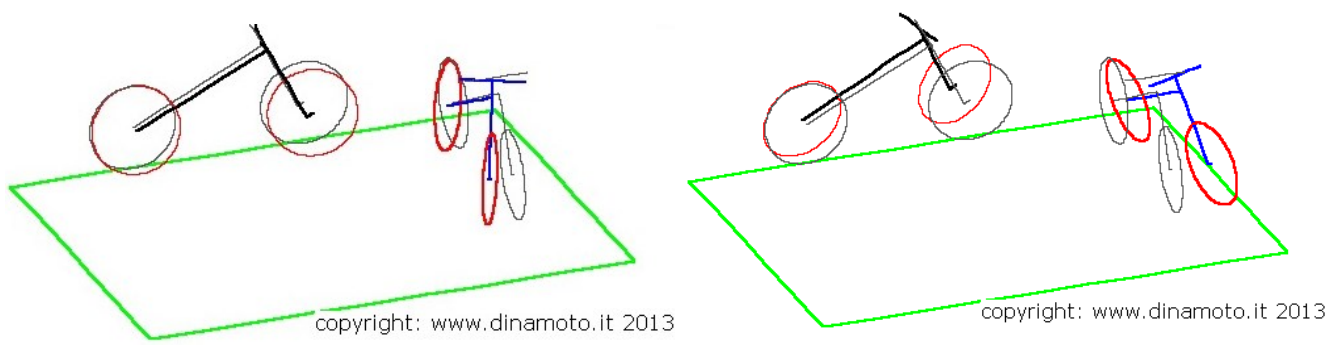

Figure 3.4 - Weave mode, by <www.dynamotion.it>.

A fourth mode is very typical in two-wheeled vehicles, but is not predicted by the model used in this work: the wobble mode, illustrated in Figure 3.5. In this mode, the front frame oscillates while the rest of the motorcycle remains in the same direction; this effect requires lateral sliding of the front wheel, which is not foreseen by the proposed model. Therefore, although this phenomenon is well observed in real motorcycles at mid-range speeds, there is not a specific pole associated with this behavior in this work.
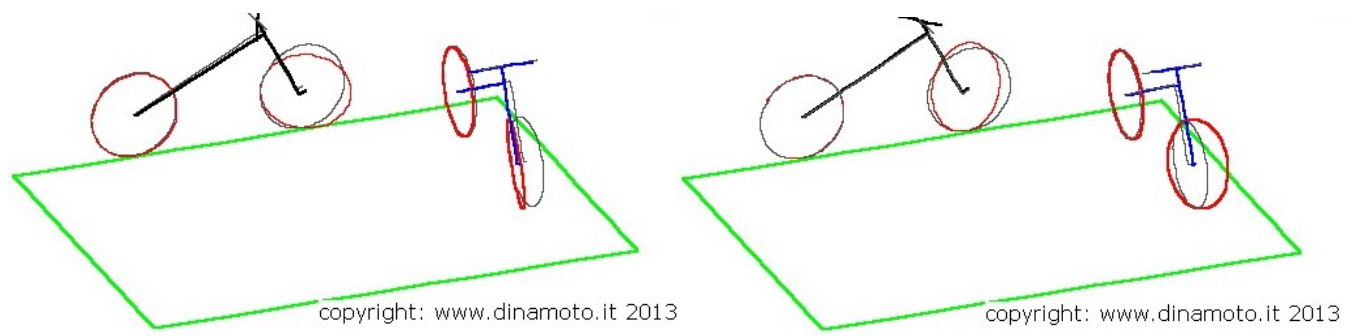

Figure 3.5 - Wobble mode, by <www.dynamotion.it $>$. 
By varying the forward speed $v_{x}$ up to $10 \mathrm{~m} / \mathrm{s}$, it is possible to analyze how the eigenvalues change its characteristics with the longitudinal speed, becoming more or less stable. Figure 3.6 separates the eigenvalues' real and imaginary parts, with an additional comparison to the benchmark bicycle, by Meijaard et al (2007); Figure 3.7 illustrates the motorcycle's result on the splane.

From the first image, it is clear that at small speeds - approximately up to $1.5 \mathrm{~m} / \mathrm{s}$ - the weave poles are purely real, indicating a non-oscillatory behavior. Since their real parts are positive, this indicates an unstable condition, which translates to falling like an inverted pendulum.

Still regarding the weave poles, after they acquire an oscillatory characteristic, their real parts tend to become more stable, i.e., less positive, with the speed increase. However, in the motorcycle's case, they are unable to effectively cross to the stable area - the negative real part of the plane. The same does not happens to the benchmark bicycle, who can acquire a stable weave mode after $4 \mathrm{~m} / \mathrm{s}$ (Hopf bifurcation). For both models, the imaginary parts increase with the vehicle's speed, which indicates higher frequency oscillations with higher velocity.

The capsize and castering modes are represented by purely real eigenvalues. The first tend to become less stable with the speed increase, since its negative module diminishes, and eventually crosses to the positive real semi-plane, becoming mildly unstable - at $12 \mathrm{~m} / \mathrm{s}$ for the motorcycle and $6 \mathrm{~m} / \mathrm{s}$ for the bicycle. The second mode, on the other hand, becomes even more stable with the speed increase, since its module moves away from the origin and positive semi-plane.

The main difference between both models is that, between 4 and $6 \mathrm{~m} / \mathrm{s}$, the benchmark bicycle model's eigenvalues all present negative real parts, which guarantees its self-stability at this speed range, i.e., the bicycle is able to keep itself in a upright position without a driver. The same phenomenon does not appears on the designed motorcycle's system, which indicates an inability to stand upright without a control strategy. Since the vehicle is originally radio controlled, this feature was probably not a concern during its design; therefore, the next chapter presents some control algorithms to work around this issue. 


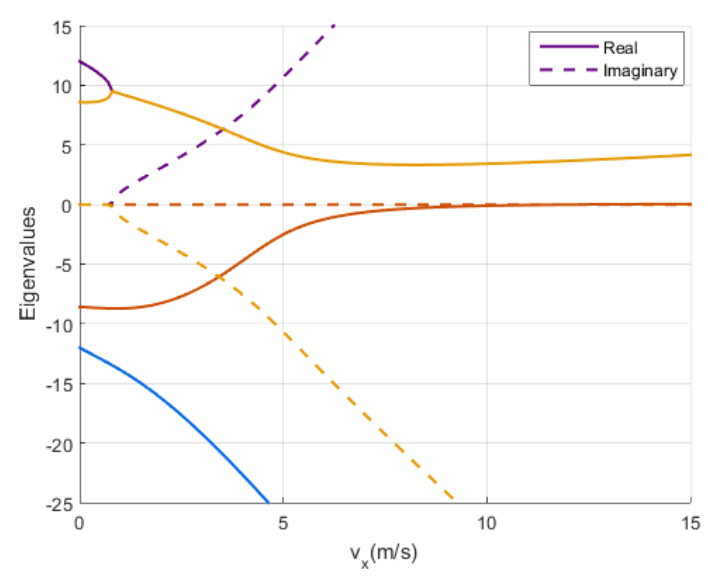

(a)

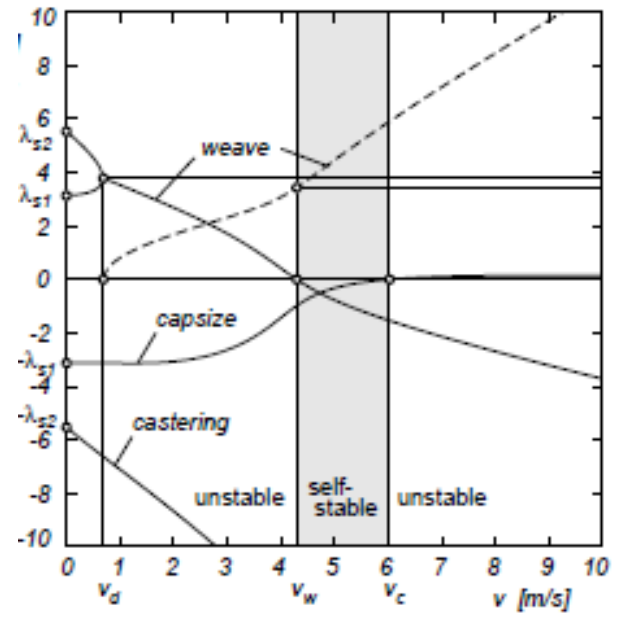

(b)

Figure 3.6 - Regions of instability and self-stability of the motorcycle with forward speed increase: (a) Motorcycle system (b) Benchmark bicycle system, Meijaard et al (2007).

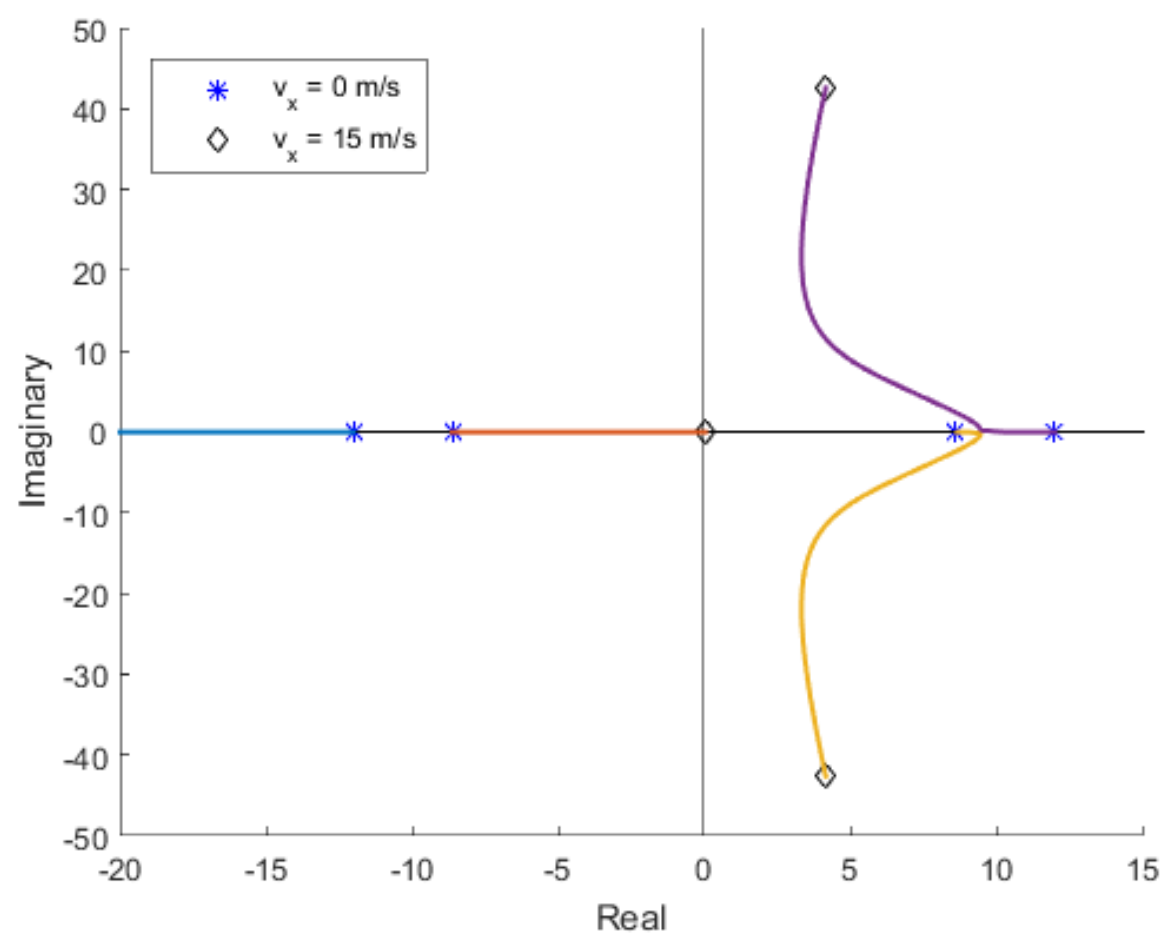

Figure 3.7 - Motorcycle's poles values with forward speed increase. 
This chapter presented a dynamic model for two-wheeled vehicles, based on the works of Sharp (1971) and Meijaard (2007). The multibody model divides the vehicle into four rigid bodies and accounts for the gyroscopic relation between them. Some simplifications are assumed, such as no tire slippage or deformation, constant longitudinal speed and small roll and yaw angles.

The parameters used were the ones experimentally determined in the previous chapter, which resulted in a fourth-order linear model with the handlebar torque as input and four state variables - the angular roll and steer speeds as well as roll and steer angles. A kinematic relation between yaw angle, lateral position and the aforementioned state variables was also included in the final state space model.

At last, the self-stability of the motorcycle was analyzed, i.e., the dynamic eigenvalue behavior that depends on the longitudinal speed, without a leaning or steering torque as input. Unlike what happens to real bicycles and motorcycles, the scale vehicle does not present a range of self-stability speed, in which the vehicle is capable of keeping upright without a rider. Therefore, the next chapter presents five different control strategies to maintain the singletrack vehicle stability and assure its trajectory. 


\section{Control strategies}

The previous chapter demonstrated how two-wheeled vehicles are capable of keeping upright without a driver, at a certain speed range, depending on its geometric design. However, in order to maintain its stability in every speed range as well as follow a determined path, an adequate control strategy is necessary.

This chapter presents five control algorithms for the proposed fourth-order linear model, based on modern control techniques (Ogata, 2010); two control strategies exclusively to maintain the roll stability, two strategies aiming to track a desired trajectory, and a fifth strategy combining both objectives. Each algorithm presents an increasing complexity, introducing the subject in a didactic order.

As seen on Chapter 3, human drivers combine two types of control to achieve this, adjusting the course via the steering system and tilting its body to avoid capsizing or to induce a roll movement. Since the studied motorcycle does not contain a mechanical counterbalance to represent the pilot's body, both stabilization and path tracking control has to be done exclusively through the steering torque. Therefore, the state space model considered for this chapter's proposed control strategies is defined by Eq. (4.1) and ((4.2), in which the lean moment $\left(T_{\varphi}\right)$ is disregarded.

$$
\begin{aligned}
& {\left[\begin{array}{c}
\ddot{\varphi} \\
\ddot{\delta} \\
\dot{\varphi} \\
\dot{\delta}
\end{array}\right]=\left[\begin{array}{cccc}
-0.48 v_{x} & -1.9 v_{x} & 75 & -14 v_{x}^{2}-13 \\
2.7 v_{x} & -3.5 v_{x} & -6.6 & 0.33 v_{x}^{2}+144 \\
1 & 0 & 0 & 0 \\
0 & 1 & 0 & 0
\end{array}\right]\left[\begin{array}{c}
\dot{\varphi} \\
\dot{\delta} \\
\varphi \\
\delta
\end{array}\right]+\left[\begin{array}{c}
-100 \\
566 \\
0 \\
0
\end{array}\right]\left[T_{\delta}\right]} \\
& {\left[\begin{array}{c}
\dot{\varphi} \\
\dot{\delta} \\
\varphi \\
\delta \\
\dot{\psi} \\
\ddot{y}_{r w}
\end{array}\right]=\left[\begin{array}{cccc}
1 & 0 & 0 & 0 \\
0 & 1 & 0 & 0 \\
0 & 0 & 1 & 0 \\
0 & 0 & 0 & 1 \\
0 & 0.09 & 0 & 1.76 v_{x} \\
0 & 0.09 v_{x} & 0 & 1.76 v_{x}^{2}
\end{array}\right]\left[\begin{array}{c}
\dot{\varphi} \\
\dot{\delta} \\
\varphi \\
\delta
\end{array}\right]+0_{6 \times 1}\left[T_{\delta}\right]}
\end{aligned}
$$




\subsection{Stability control through state feedback}

The first step to an autonomous motorcycle is to balance itself; considering all state variables ideally measurable, it is possible to make a full state feedback in order to guarantee the vehicle's stability, as depicted in Figure 4.1, based on Eq. (4.1). The considered model is completely observable and controllable, to any given longitudinal speed. Since the control input is defined by Eq. (4.3) and considering the desired state variables as null - aiming to achieve zero roll and steer angular speeds and positions -, Eq. (4.4) brings the new state space model for the system.

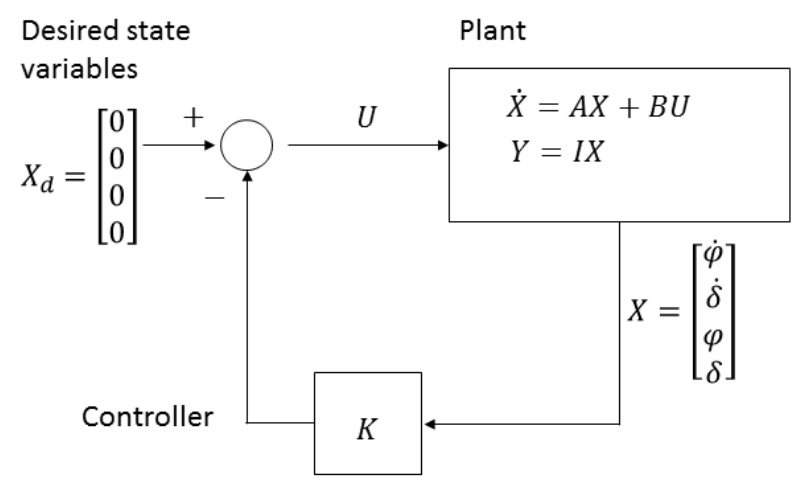

Figure 4.1 - Block diagram for stability control through state feedback.

$U=X_{d}-K X==-\left[\begin{array}{llll}K_{1} & K_{2} & K_{3} & K_{4}\end{array}\right]\left[\begin{array}{c}\dot{\varphi} \\ \dot{\delta} \\ \varphi \\ \delta\end{array}\right]$

$\dot{X}=(A-B K) X$

The new state space model will have eigenvalues depending on the chosen feedback gain; once the system is completely controllable, it is possible to change these poles through and adequate gain set, a technique known as pole placement.

Considering three different constant speeds $(5,10$ and $15 \mathrm{~m} / \mathrm{s})$, the state feedback controller is designed to keep the poles' absolute values while maintaining them at the negative quadrant of the s-plane. Table 4.1 brings the feedback gain vectors to the same desired closed-loop poles: $\left[\begin{array}{llll}-10 & -15 & -20 & -25\end{array}\right]$, chosen in order to keep the stabilization time under one second with overdamped characteristics. 
It is important to note that, for every control strategy, the steer angle is limited at -20 and 25 degrees (or -0.35 and 0.44 radians) and the steering torque, at $0.32 \mathrm{~N}-\mathrm{m}$, the servo maximum torque.

Figures 4.2 and 4.3 illustrate the simulation results to an initial condition of $0.5 \mathrm{rad} / \mathrm{s}$ roll angular speed: both angular speeds stabilize at zero, guaranteeing the system's stability; the roll and steer angles reach a null steady state value while keeping the small angle assumption for the model, so the stabilization criteria for the state feedback controller has been met. At last, the torque input calculated via the controller is under the servo limit, i.e., under $0.32 \mathrm{~N}-\mathrm{m}$.

Table 4.1 - State feedback gain to three different speeds.

\begin{tabular}{lllll}
\hline Speed $(\mathbf{m} / \mathbf{s})$ & \multicolumn{5}{l}{ State feedback gain $\boldsymbol{K}$} \\
\hline 5 & {$\left[\begin{array}{lllll}-0.07 & 0.08 & -0.82 & 2.25\end{array}\right]$} \\
10 & {$\left[\begin{array}{lllll}0.02 & 0.06 & -0.24 & 2.91\end{array}\right]$} \\
15 & {$\left[\begin{array}{llll}0.06 & 0.03 & -0.12 & 3.21\end{array}\right]$} \\
\hline
\end{tabular}
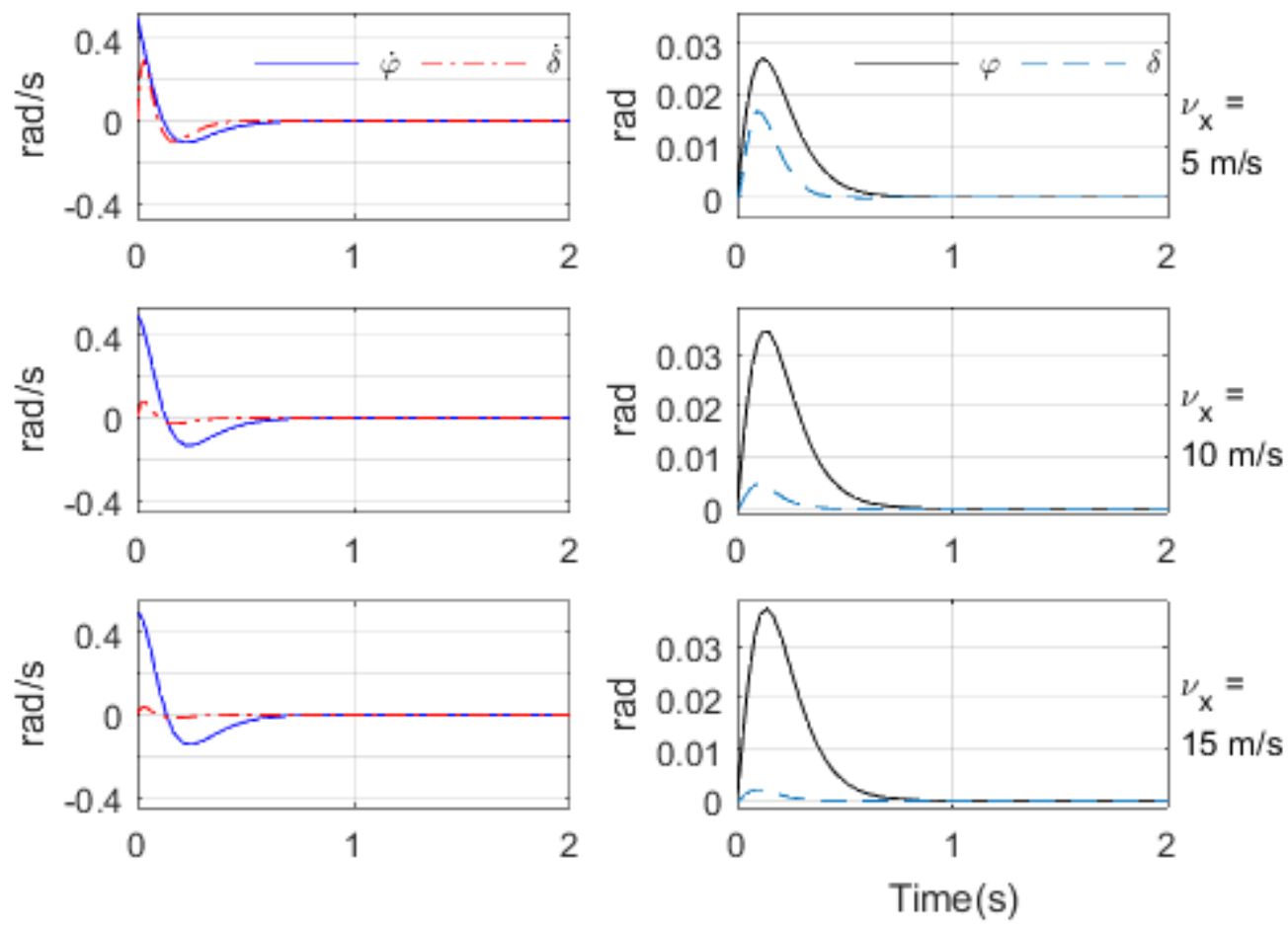

Figure 4.2 - Angular speed and position results for stability control through state feedback. 


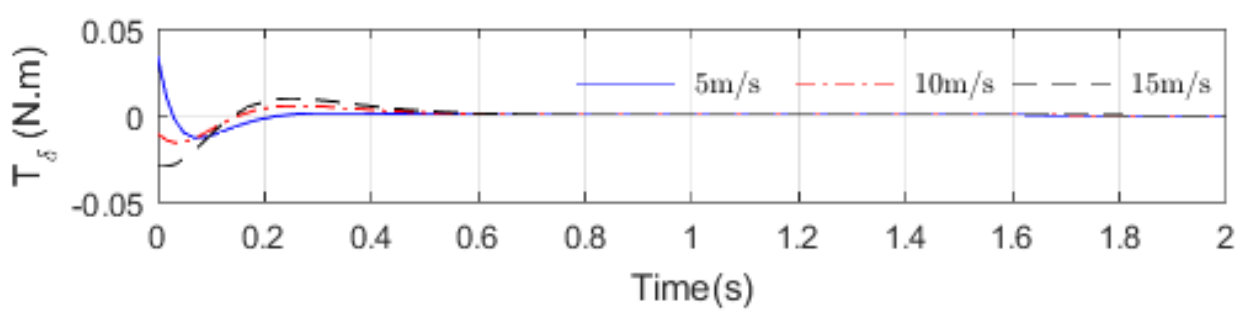

Figure 4.3 - Torque input for stability control through state feedback.

\subsection{Stability control through observer feedback}

In experimental setups, it is common to not have all sensors available; on the studied motorcycle, only the steer angle $(\delta)$, given by the potentiometer, and the roll angular speed $(\dot{\varphi})$, measured by a gyrometer, are directly measurable. Therefore, it is necessary to have a state observer estimating the missing variables in order to calculate the controller input.

Since the main objective is to stabilize the motorcycle, the model used in this section is represented by Eq. (4.5); the model has been reorganized in order to separate the measured variables from the estimated ones. Since the new system is completely observable, at any given speed, it is possible to build an adequate observer such as described in Figure 4.4.

$$
\begin{aligned}
& {\left[\begin{array}{c}
\ddot{\varphi} \\
\dot{\delta} \\
\dot{\varphi} \\
\ddot{\delta}
\end{array}\right]=\left[\begin{array}{cc:cc}
-0.48 v_{x} & -14 v_{x}^{2}-13 & 75 & -1.9 v_{x} \\
0 & 0 & 0 & 1 \\
\hdashline 1 & 0 & 0 & 0 \\
2.7 v_{x} & 0.33 v_{x}^{2}+144 & -6.6 & -3.5 v_{x}
\end{array}\right]\left[\begin{array}{c}
\dot{\varphi} \\
\delta \\
\varphi \\
\dot{\delta}
\end{array}\right]+\left[\begin{array}{c}
-100 \\
0 \\
\hdashline 0 \\
066
\end{array}\right]\left[T_{\delta}\right]} \\
& Y=\left[\begin{array}{llll}
1 & 0 & 0 & 0 \\
0 & 1 & 0 & 0
\end{array}\right]\left[\begin{array}{c}
\dot{\varphi} \\
\delta \\
-\bar{\varphi} \\
\dot{\delta}
\end{array}\right]+0_{2 \times 1}\left[T_{\delta}\right]
\end{aligned}
$$

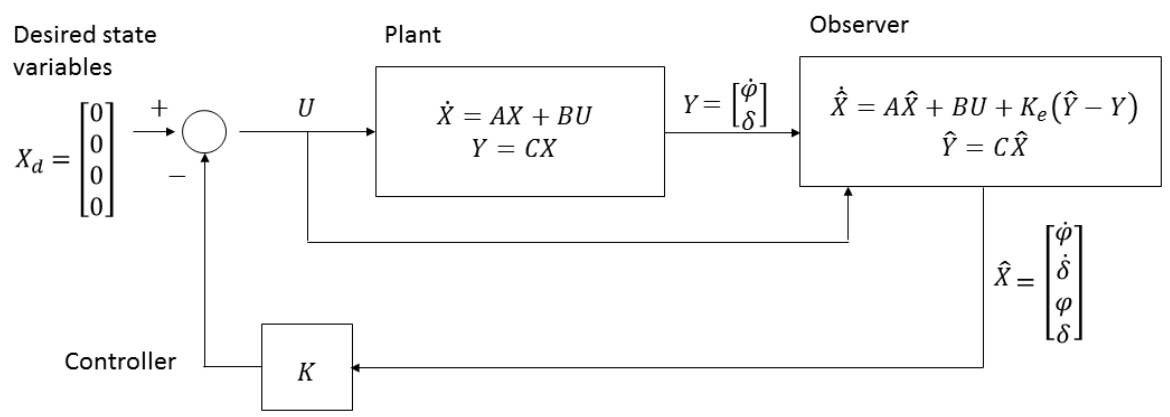

Figure 4.4 - Block diagram for stability control through observer feedback. 
Provided that the observer is estimating only the variables not directly measured, i.e., the steer angular speed and the roll angle, it is classified as a reduced-order observer. Eq. (4.6) and (4.7) bring, respectively, a generic statespace and observer model, with $x_{a}$ being the $m$ measured variables and $x_{b}$ the $n$ observed ones (Ogata, 2010). Equation (4.8 describes the generic observer state-space model, which gives the observer poles and allows to calculate the gain matrix $\left(K_{e}\right)$ through pole placement.

$$
\begin{aligned}
& {\left[\begin{array}{l}
\dot{x}_{a} \\
\dot{x}_{b}
\end{array}\right]=\left[\begin{array}{ll}
A_{a a} & A_{a b} \\
A_{b a} & A_{b b}
\end{array}\right]\left[\begin{array}{l}
x_{a} \\
x_{b}
\end{array}\right]+\left[\begin{array}{l}
B_{a} \\
B_{b}
\end{array}\right] U} \\
& Y=\left[\begin{array}{ll}
I_{n \times n} & 0_{n \times m}
\end{array}\right]\left[\begin{array}{l}
x_{a} \\
x_{b}
\end{array}\right] \\
& \dot{\hat{\eta}}=\hat{A} \hat{\eta}+\hat{B} Y+\hat{F} U \\
& \quad=\left(A_{b b}-K_{e} A_{a b}\right) \hat{\eta}+\left(\hat{A} K_{e}+A_{b a}-K_{e} A_{a a}\right) Y+\left(B_{b}-K_{e} B_{a}\right) U \\
& \hat{x}=\hat{C} \hat{\eta}+\widehat{D} Y=\left[\begin{array}{c}
0_{n \times n} \\
I_{m \times m}
\end{array}\right] \hat{\eta}+\left[\begin{array}{c}
I_{n \times n} \\
K_{e}
\end{array}\right] Y \\
& \dot{\hat{x}}_{b}-\dot{x}_{b}=\left(A_{b b}-K_{e} A_{a b}\right) \underbrace{\left(\hat{x}_{b}-x_{b}\right)}_{\eta}
\end{aligned}
$$

With the motorcycle state-space model presented in Eq. (4.5) and the previous generic observer equations, it is possible to build the motorcycle observer, defined in Eq. (4.9). It is important to emphasize that only the roll angle and steer singular speed being estimated by the observer $(\hat{\eta})$, this means the final observed state variable $(\hat{X})$ will contain both the measured and estimated variables.

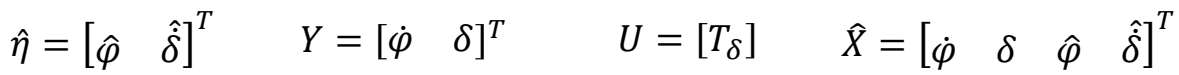

$$
\begin{aligned}
& \hat{A}=\left[\begin{array}{cc}
0 & 0 \\
-6.6 & -3.5 v_{x}
\end{array}\right]-K_{e_{2 \times 2}}\left[\begin{array}{cc}
75 & -1.9 v_{x} \\
0 & 1
\end{array}\right] \\
& \hat{B}=\hat{A} K_{e_{2 \times 2}}+\left[\begin{array}{cc}
1 & 0 \\
2.7 v_{x} & 0.33 v_{x}^{2}+144
\end{array}\right]-K_{e_{2 \times 2}}\left[\begin{array}{cc}
-0.48 v_{x} & -14 v_{x}^{2}-13 \\
0 & 0
\end{array}\right] \\
& \hat{F}=\left[\begin{array}{c}
0 \\
566
\end{array}\right]-K_{e_{2 \times 2}}\left[\begin{array}{c}
-100 \\
0
\end{array}\right] \quad \hat{C}=\left[\begin{array}{l}
0_{2 \times 2} \\
I_{2 \times 2}
\end{array}\right] \quad \widehat{D}=\left[\begin{array}{c}
I_{2 \times 2} \\
K_{e_{2 \times 2}}
\end{array}\right]
\end{aligned}
$$

Eq. (4.10) presents the feedback control equation; since the observer $\left(K_{e}\right)$ and feedback $(K)$ gains are independent, it is possible to choose two different sets of desired eigenvalues for the final closed loop system, the observer and 
the state feedback poles. The state feedback follows the same logic applied to the previous control algorithm: yielding a stable and non-oscillatory time response, by keeping the state poles at the negative and real side of the $s$ plane. The same set of desired poles was used, $\left[\begin{array}{llll}-10 & -15 & -20 & -25\end{array}\right]$; therefore, the state feedback gain $K$ is the same as presented in Table 4.1, adjusting the gain vector order to the new state variable.

$U=X_{d}-K \hat{X}==-\left[\begin{array}{llll}K_{1} & K_{4} & K_{3} & K_{2}\end{array}\right]\left[\begin{array}{c}\dot{\varphi} \\ \delta \\ \hat{\varphi} \\ \hat{\dot{\delta}}\end{array}\right]$

Usually, the observer poles are designed to have a dynamic faster than the system's (Ogata, 2010); in this work, since only two variables are being estimated, it is necessary to choose two desired poles. Thus, the observer eigenvalues are set to be three times faster than the state poles, i.e., they are equal to the lowest real part of the feedback poles multiplied by three. The observer gain is calculated through pole placement, using Eq. (4.8), where the observer state matrix $A$ is highlighted. The observer desired eigenvalues and gain to three different speeds is detailed at Table 4.2.

For comparison between control strategies performance, the same conditions are applied to the system simulation: an initial condition of $0.5 \mathrm{rad} / \mathrm{s}$ roll angular speed, with steer angle saturation at 20 degrees and steer torque up to $0.32 \mathrm{~N}-\mathrm{m}$. Figure 4.5 illustrates the angles and angular speeds behavior: though less oscillatory than the previous control strategy, the roll angle $\varphi-$ determined by the observer - peaks to high values; the worst case is at the highest speed, where it reaches a maximum of 0.2 rad or 11 degrees, approximately, which maintains the small angles hypothesis.

The steer angle varies to a maximum of $0.03 \mathrm{rad}$ (1.7 degrees). Figure 4.6 shows the torques applied to the system's handlebar, all being smaller than the servo saturation torque. Briefly, the observer feedback controller presents a less damped time response, when compared to the full state feedback, with higher roll amplitudes, though it is in conformity with the small angles hypothesis and servo saturation, while also keeping the roll and steer angles null at the steady state. 
Table 4.2 - Observer feedback gain to three different speeds.

\begin{tabular}{lll}
\hline Speed $(\mathbf{m} / \mathbf{s})$ & Desired eigenvalues & Observer feedback gain $\boldsymbol{K}_{\boldsymbol{e}}$ \\
\hline 5 & {$\left[\begin{array}{ll}-30 & -45\end{array}\right]$} & {$\left[\begin{array}{cc}0.40 & 3.80 \\
-0.09 & 26.67\end{array}\right]$} \\
10 & {$\left[\begin{array}{ll}-30 & -45\end{array}\right]$} & {$\left[\begin{array}{cc}0.40 & 7.60 \\
-0.09 & 8.33\end{array}\right]$} \\
15 & {$\left[\begin{array}{ccc}-30 & -45\end{array}\right]$} & {$\left[\begin{array}{cc}0.40 & 11.40 \\
-0.09 & -10.01\end{array}\right]$} \\
\hline
\end{tabular}
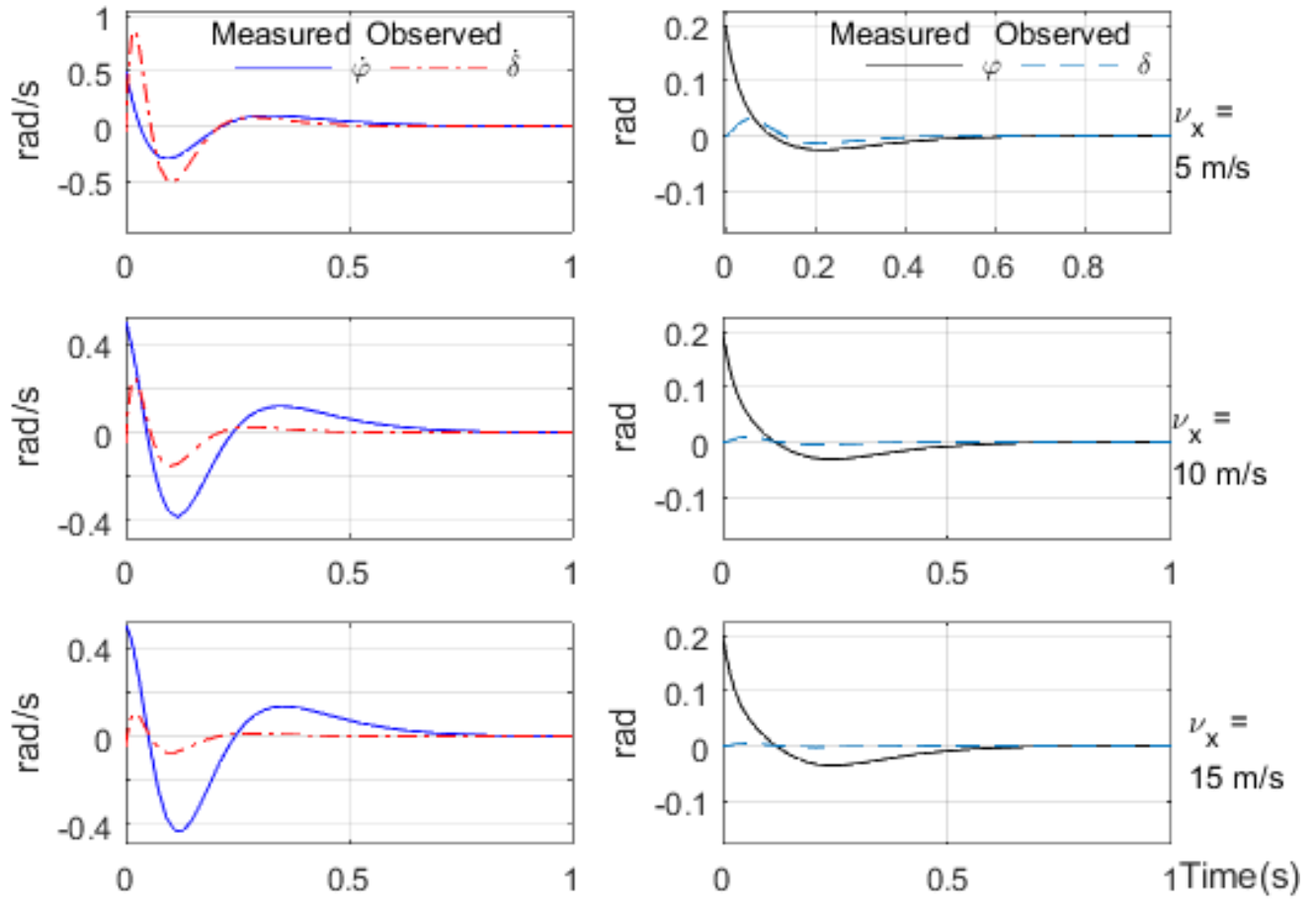

Figure 4.5 - Angular speed and position results for stability control through observer feedback.

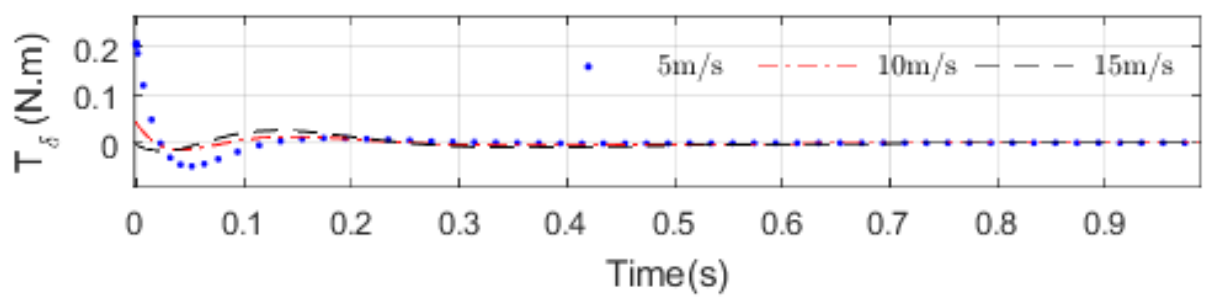

Figure 4.6 - Torque input for stability control through observer feedback. 


\subsection{Path tracking through state feedback}

The next step to control an autonomous motorcycle is to make it follow a desired trajectory, at any given speed. The mathematical model used to achieve this goal is a modification of the one presented in Eq. (4.1) and (4.2), in which the motorcycle yaw angle $(\psi)$, lateral velocity $\left(\dot{y}_{r w}\right)$ and position $\left(y_{r w}\right)$ are included. In order to avoid the double integration from the lateral acceleration, the model is rewritten in Eq. (4.11) and (4.12), with the yaw angle and lateral position as new state variables.

$$
\begin{aligned}
& {\left[\begin{array}{c}
\ddot{\varphi} \\
\ddot{\delta} \\
\dot{\varphi} \\
\dot{\delta} \\
\dot{\psi} \\
\dot{y}_{r w}
\end{array}\right]=\left[\begin{array}{cccccc}
-0.48 v_{x} & -1.9 v_{x} & 75 & -14 v_{x}^{2}-13 & 0 & 0 \\
2.7 v_{x} & -3.5 v_{x} & -6.6 & 0.33 v_{x}^{2}+144 & 0 & 0 \\
1 & 0 & 0 & 0 & 0 & 0 \\
0 & 1 & 0 & 0 & 0 & 0 \\
0 & 0.09 & 0 & 1.76 v_{x} & 0 & 0 \\
0 & 0 & 0 & 0 & v_{x} & 0
\end{array}\right]\left[\begin{array}{c}
\dot{\varphi} \\
\dot{\delta} \\
\varphi \\
\delta \\
\psi \\
y_{r w}
\end{array}\right]+\left[\begin{array}{c}
-100 \\
566 \\
0 \\
0 \\
0 \\
0
\end{array}\right]\left[T_{\delta}\right]} \\
& \mathrm{Y}=\left[\begin{array}{llllll}
1 & 0 & 0 & 0 & 0 & 0 \\
0 & 1 & 0 & 0 & 0 & 0 \\
0 & 0 & 1 & 0 & 0 & 0 \\
0 & 0 & 0 & 1 & 0 & 0 \\
0 & 0 & 0 & 0 & 1 & 0 \\
0 & 0 & 0 & 0 & 0 & 1
\end{array}\right]\left[\begin{array}{c}
\dot{\varphi} \\
\dot{\delta} \\
\varphi \\
\delta \\
\psi \\
y_{r w}
\end{array}\right]+0_{6 \times 1}\left[T_{\delta}\right]
\end{aligned}
$$

Considering all variables ideally measured, it is possible to feedback the state signals in order to guarantee both the motorcycle stability and trajectory; Figure 4.7 brings the block diagram to the state feedback. This feedback strategy is only possible because the system is completely controllable.

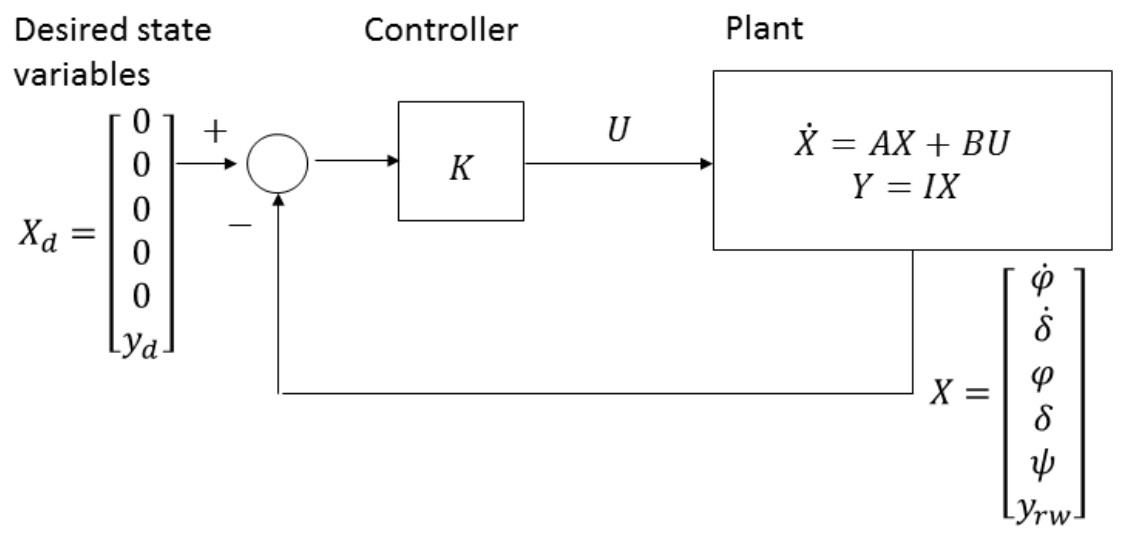

Figure 4.7 - Block diagram for path tracking through output feedback. 
Eq. (4.13) illustrates the controller definition, in which the input torque becomes the desired trajectory minus a proportional gain to the state variables, and Eq. (4.14) shows the feedback state-space model, considering the $D$ matrix null.

$U=K\left(X_{d}-X\right)=\left[\begin{array}{llllll}K_{1} & K_{2} & K_{3} & K_{4} & K_{5} & K_{6}\end{array}\right]\left[\begin{array}{c}\dot{\varphi} \\ \dot{\delta} \\ \varphi \\ \delta \\ \psi \\ y_{d}-y_{r w}\end{array}\right]$

$\dot{X}=(A-B K) X+B K X_{d}$

$\dot{X}=(A-B K) X+B K_{6} y_{d}$

Like the previous control strategies, the desired eigenvalues are the same to every speed simulated, in order to guarantee an equal time response at different speeds. The control gain vector $K$, is once again defined by the pole placement technique; Table 4.3 brings the feedback gain vector $(K)$ for three different speeds, considering the desired eigenvalues as $\left[\begin{array}{llllll}-10 & -15 & -20 & -25 & -30 & -35\end{array}\right]$.

Table 4.3 - Path tracking state feedback gain to three different speeds.

\begin{tabular}{lllllll}
\hline Speed $(\mathbf{m} / \mathbf{s})$ & \multicolumn{2}{l}{ State feedback gain $\boldsymbol{l}$} \\
\hline 5 & {$\left[\begin{array}{lllllll}-2.09 & -0.17 & -18.25 & -10.56 & -66.57 & -43.24\end{array}\right]$} \\
10 & {$\left[\begin{array}{llllll}-0.55 & 0.07 & -5.18 & 0.50 & -33.86 & -10.81\end{array}\right]$} \\
15 & {$\left[\begin{array}{llllll}-0.21 & 0.10 & -2.42 & 4.67 & -22.70 & -4.80\end{array}\right]$} \\
\hline
\end{tabular}

The simulation executed with this control algorithm was a double lane change, a common trajectory when avoiding obstacles. The desired path had a lateral displacement of one meter with a slope, to fit the small angle and displacement hypothesis. Figure 4.8 illustrates a comparison between the path traveled in each velocity, in which the counter-steering effect is slightly perceptible, with the simulated path initially going against the desired path and latter aligning to the same direction. The motorcycle trajectory is delayed in comparison to the path input, with a maximum error of 0.237 meters at the highest speed, or approximately $24 \%$ of the maximum trajectory. Figure 4.9 compares the input torque in each simulation and Figure 4.10 describes the roll, steer and yaw angular speed and positions; all cited variables are under the saturation limits and small angle hypothesis. 


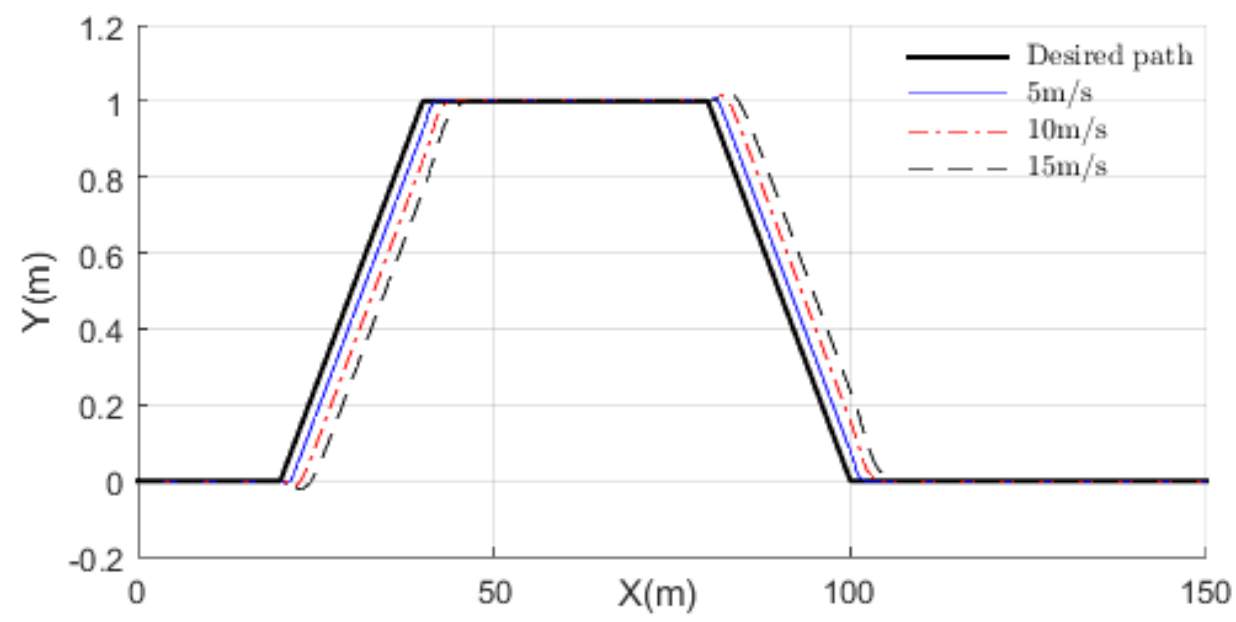

Figure 4.8 -Position results for path tracking through state feedback.

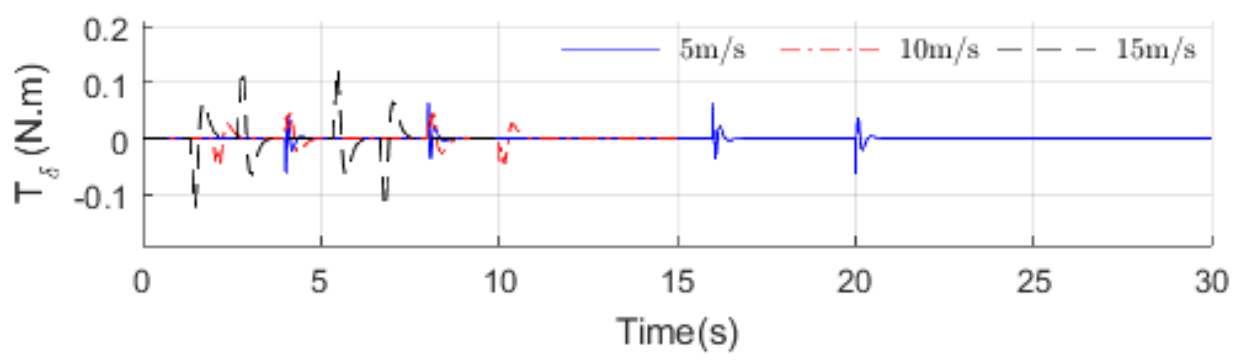

Figure 4.9 - Torque input for path tracking through state feedback.
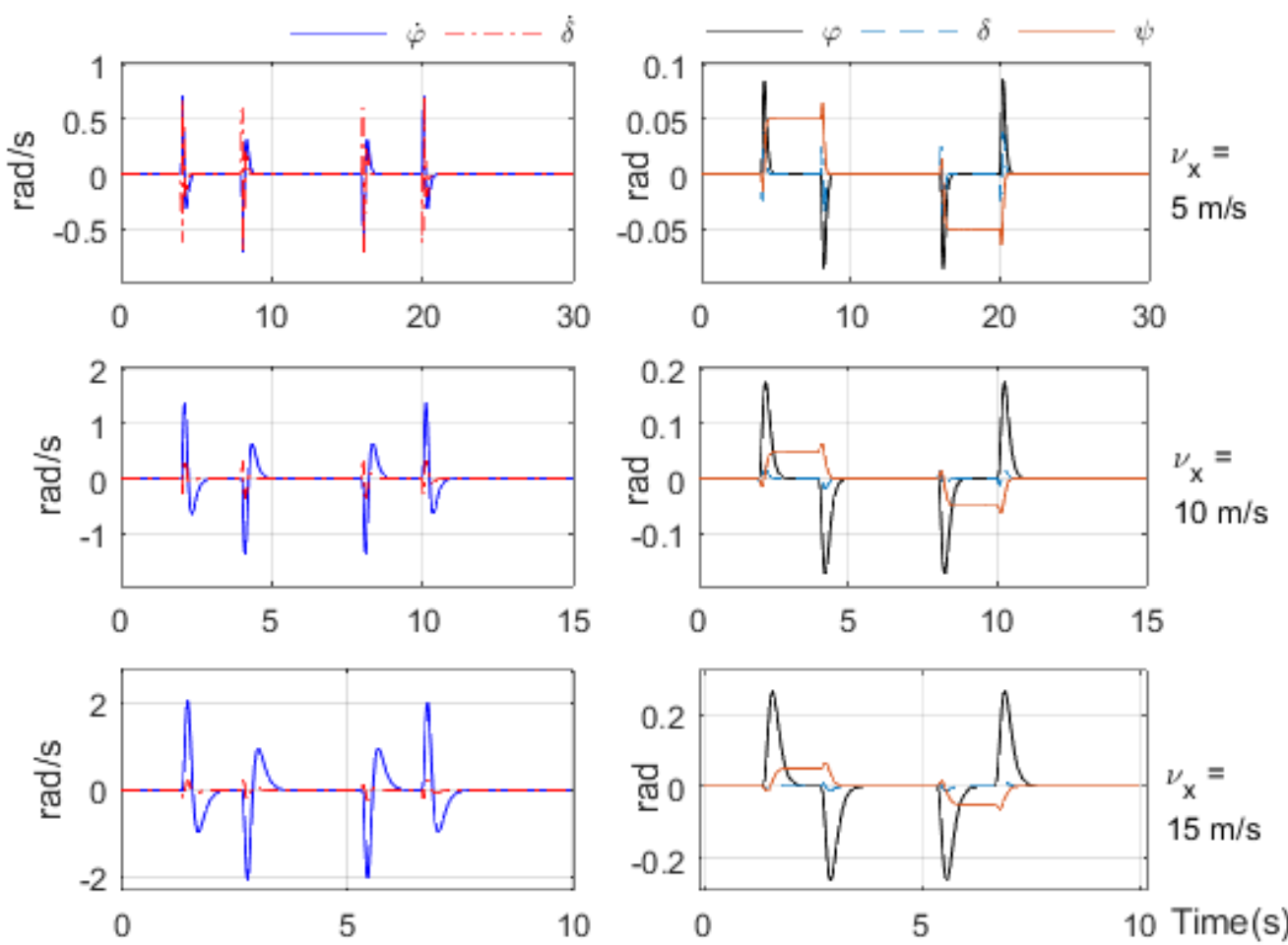

Figure 4.10 - Angular speed and position results for path tracking through state feedback. 


\subsection{Path tracking through linear quadratic regulator}

Another interesting control strategy applied to the path tracking problem is the linear quadratic regulator, in which the feedback control gain matrix is calculated in order to minimize a performance index, instead of the poleplacement method.

The steering torque (input) is still proportional to the state variables - Eq. (4.15) - but the gain matrix $K_{l}$ minimizes the cost function presented in Eq. (4.16). The parameters of matrices $Q$ and $R$ are designed to penalize deviations on the state variables and control signals, respectively; since all variables are considered independent, these matrices become diagonal weights.

$U=K_{l}\left(X_{d}-X\right)=\left[\begin{array}{llllll}K_{l 1} & K_{l 2} & K_{l 3} & K_{l 4} & K_{l 5} & K_{l 6}\end{array}\right]\left(X_{d}-\left[\begin{array}{c}\dot{\varphi} \\ \dot{\delta} \\ \varphi \\ \delta \\ \psi \\ y_{r w}\end{array}\right]\right)$

$J=\int_{0}^{\infty}\left(x^{T} Q x+u^{T} R u\right) d t$

In this control approach, higher gains - or penalties - were assigned to the roll angle, steer angle and, mainly, lateral displacement, in order to guarantee they are closer to the desired reference value. The gains were adjusted after simulations aiming to keep the minimum path error, which was around $30 \%$ of the total course. Eq. (4.17) brings the matrices used in all speeds simulated, where it resulted in different closed loop poles, explicit in Table 4.4.

$\begin{array}{ccccccc}Q_{\dot{\varphi}} & Q_{\dot{\delta}} & Q_{\varphi} & Q_{\delta} & Q_{\psi} & Q_{y_{r w}} & \boldsymbol{R} \\ 0.1 & 0.1 & 0.5 & 0.5 & 0.1 & 100 & \mathbf{0 . 1}\end{array}$

Table 4.4 - Linear quadratic regulator gain to three different speeds.

\begin{tabular}{|c|c|c|c|c|c|c|c|c|}
\hline $\begin{array}{l}\text { Speed } \\
(\mathrm{m} / \mathrm{s})\end{array}$ & $\begin{array}{l}\text { Closed loop } \\
\text { eigenvalues }\end{array}$ & & LQR g & in $K_{l}$ & & & & \\
\hline 5 & {$\left[\begin{array}{l}-4.7 \pm 5.4 i \\
-15 \pm 11 i\end{array}\right.$} & $\left.\begin{array}{c}-5.7 \\
-577\end{array}\right]$ & {$[-3.45$} & 0.44 & -28.7 & 3.79 & -68.7 & $-31.6]$ \\
\hline 10 & {$\left[\begin{array}{l}-4.8 \pm 5.3 i \\
-28 \pm 25 i\end{array}\right.$} & $\left.\begin{array}{l}-5.6 \\
-577\end{array}\right]$ & {$[-2.85$} & 0.56 & -23.5 & 9.69 & -126.3 & $-31.6]$ \\
\hline 15 & {$\left[\begin{array}{l}-4.7 \pm 5.3 i \\
-41 \pm 39 i\end{array}\right.$} & $\left.\begin{array}{l}-5.6 \\
-577\end{array}\right]$ & {$[-2.66$} & 0.61 & -21.9 & 15.6 & -184.2 & $-31.6]$ \\
\hline
\end{tabular}


With the stable poles from the linear quadratic regulator, it is possible to apply a desired double lane change to the motorcycle's lateral displacement. Figure 4.11 brings the resulting paths, in which it is clear the vehicle is capable of following the desired trajectory, but with greater delay than the previous control strategy. Figure 4.12 describes the torque input applied to the system, with amplitude within the servomotor's saturation limits, $0.32 \mathrm{~N}-\mathrm{m}$; Figure 4.13 illustrates the behavior of angular speeds and position, all maintaining the small angles hypothesis, i.e., under 0.2 rad or 11 degrees.

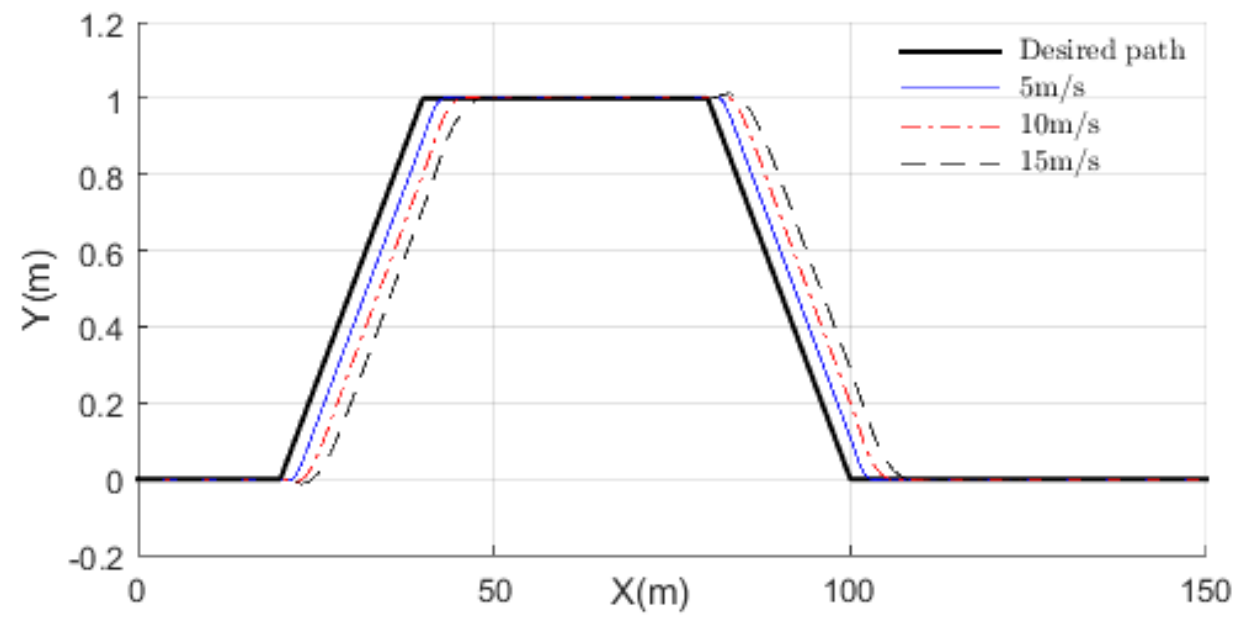

Figure 4.11 - Position results for path tracking through LQR.

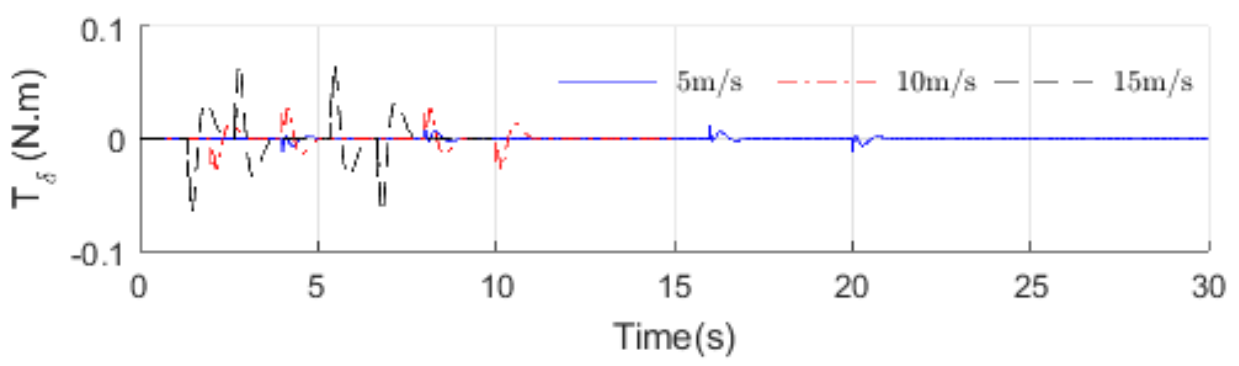

Figure 4.12 - Torque input for path tracking through LQR. 

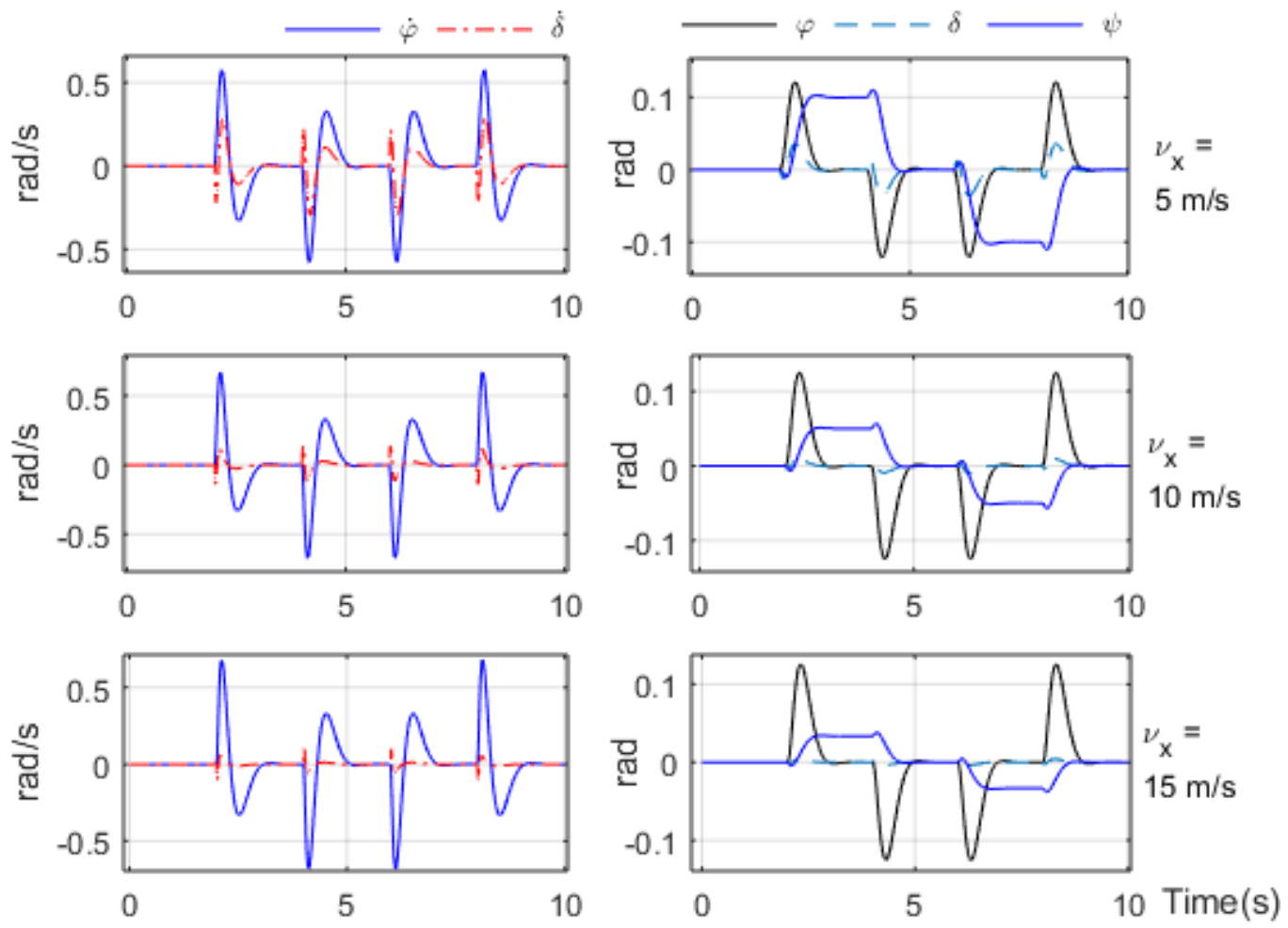

Figure 4.13 - Angular speed and position for path tracking through LQR.

\subsection{Path tracking through trajectory preview}

At last, the final control strategy applied to the motorcycle system is the path tracking through trajectory preview (Dao, Chen; 011). The previous strategies were passively responding to changes on the target path: once the error is considerably, the vehicle is actuated in order to steer closer to the trajectory goal. In real riding experience, the human driver looks forward at a preview point and adjust its trajectory aiming at future positions.

Unlike four-wheeled vehicles, path tracking for motorcycles has to take into account the roll stability, in addition to the planar motion information such as position, speed and orientation. Therefore, this control strategy is divided in two parts: a roll stability and trajectory controllers. For the roll angle control, a full state feedback was used - similar to the one depicted in Section 4.2 - and is illustrated in Figure 4.14. 


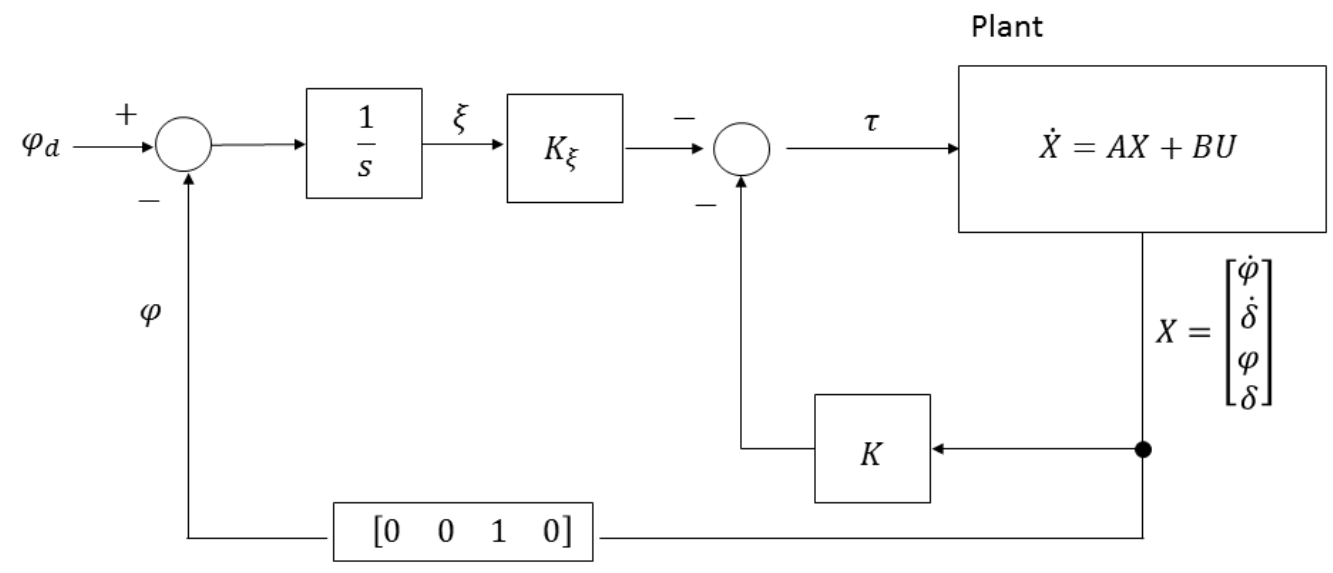

Figure 4.14 - Block diagram for roll angle control.

The roll angle control is composed of two loops: a state feedback control (inner loop) and an integral control between the error comparator and the plant. This integrator was added to attenuate the effect of external disturbances. The control law is described in Eq. (4.18), where $K$ is a vector gain with the same dimension as the state variable (Eq. (4.3)), $K_{\xi}$ is a constant control gain and $\xi$ is the integral of the roll tracking error (Eq. (4.19)).

$\tau=-K X-K_{\xi} \xi$

$\dot{\xi}=\varphi_{d}-\varphi=\varphi_{d}-\tilde{C} X$

Considering a full state feedback, the $C$ matrix is equal to the identity matrix and the $\tilde{C}=\left[\begin{array}{llll}0 & 0 & 1 & 0\end{array}\right] C$ matrix is the third row of $C$, where the value of the roll angle is located. A new state space model can be derived from adding the tracking error to the state variables, as exemplified in Eq. (4.20). The input torque can then be calculated as a feedback from the new state space model (Eq. (4.21)).

$$
\begin{aligned}
& {\left[\begin{array}{l}
\dot{X} \\
\dot{\xi}
\end{array}\right]=\left[\begin{array}{cc}
A & 0 \\
-\tilde{C} & 0
\end{array}\right]\left[\begin{array}{l}
X \\
\xi
\end{array}\right]+\left[\begin{array}{l}
B \\
0
\end{array}\right] \tau+\left[\begin{array}{l}
0 \\
1
\end{array}\right] \varphi_{d}} \\
& \tau=-\widetilde{K}\left[\begin{array}{l}
X \\
\xi
\end{array}\right]=-\left[\begin{array}{ll}
K_{1 \times 4} & K_{\xi}
\end{array}\right]\left[\begin{array}{l}
X \\
\xi
\end{array}\right]
\end{aligned}
$$


The gain vector $\widetilde{K}$ can be determined through the pole placement technique, considering the new $\tilde{A}$ and $\tilde{B}$ matrices. Since the new state space matrix has a null line, the additional pole to the open loop system is equal to zero, which could cause instabilities. All speeds have the same desired eigenvalues, $\left[\begin{array}{lllll}-10 & -15 & -20 & -25 & -30\end{array}\right]$, and the resulting feedback gain vector is at Table 4.5.

Table 4.5 - Roll control feedback gain to three different speeds.

\begin{tabular}{llllll}
\hline Speed $(\mathbf{m} / \mathbf{s})$ & \multicolumn{6}{l}{ Roll control feedback gain $(\widetilde{\mathbf{K}})$} \\
\hline 5 & {$\left[\begin{array}{llllll}-0.24 & 0.10 & -3.58 & 3.32 & 11.55\end{array}\right]$} \\
10 & {$\left[\begin{array}{lllll}-0.03 & 0.10 & -1.02 & 5.13 & 2.81\end{array}\right]$} \\
15 & {$\left[\begin{array}{lllll}0.03 & 0.08 & -0.48 & 5.89 & 1.24\end{array}\right]$} \\
\hline
\end{tabular}

To validate the analytical results, the motorcycle's model was simulated following a sinusoidal reference roll angle with 0.2 rad (11 degrees) amplitude and $0.16 \mathrm{~Hz}$ (1 rad/s) frequency. The result can be observed on Figure 4.15, which brings the comparison between target and simulated roll angle; in all three speeds, the roll angle behavior was the same, with a maximum error of 0.05 rad ( 3 degrees) or $28 \%$ of the total amplitude. In Figure 4.17 it is possible to notice that at higher speeds, it is necessary a smaller steer angle to obtain the same roll angle, which is corroborated by the torque behavior, at Figure 4.16.

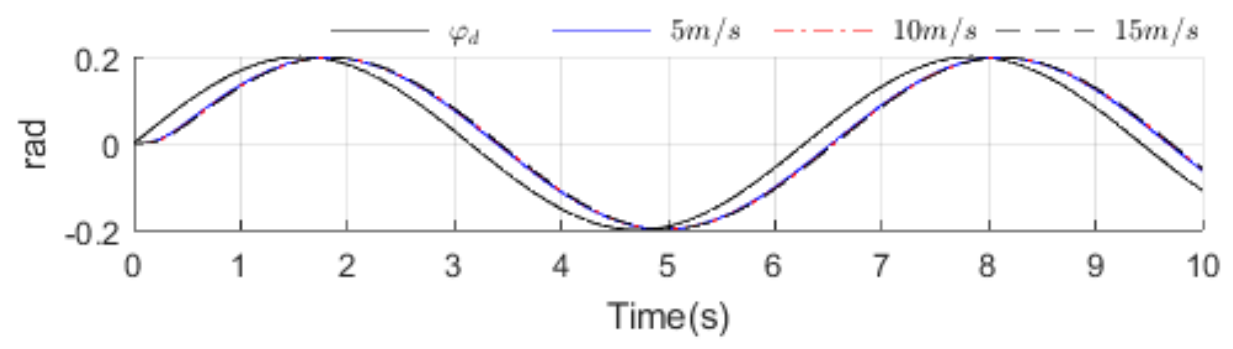

Figure 4.15 - Roll angles results for roll control through path preview.

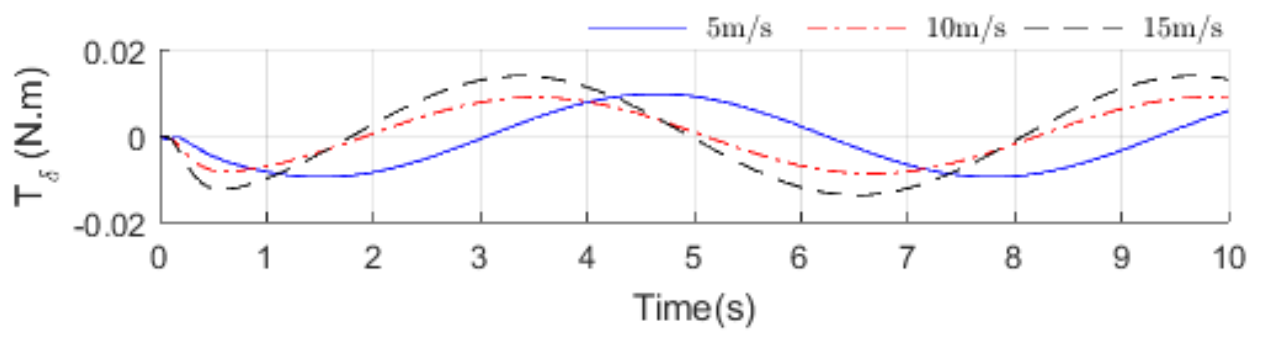

Figure 4.16 - Torque input for roll control through path preview. 

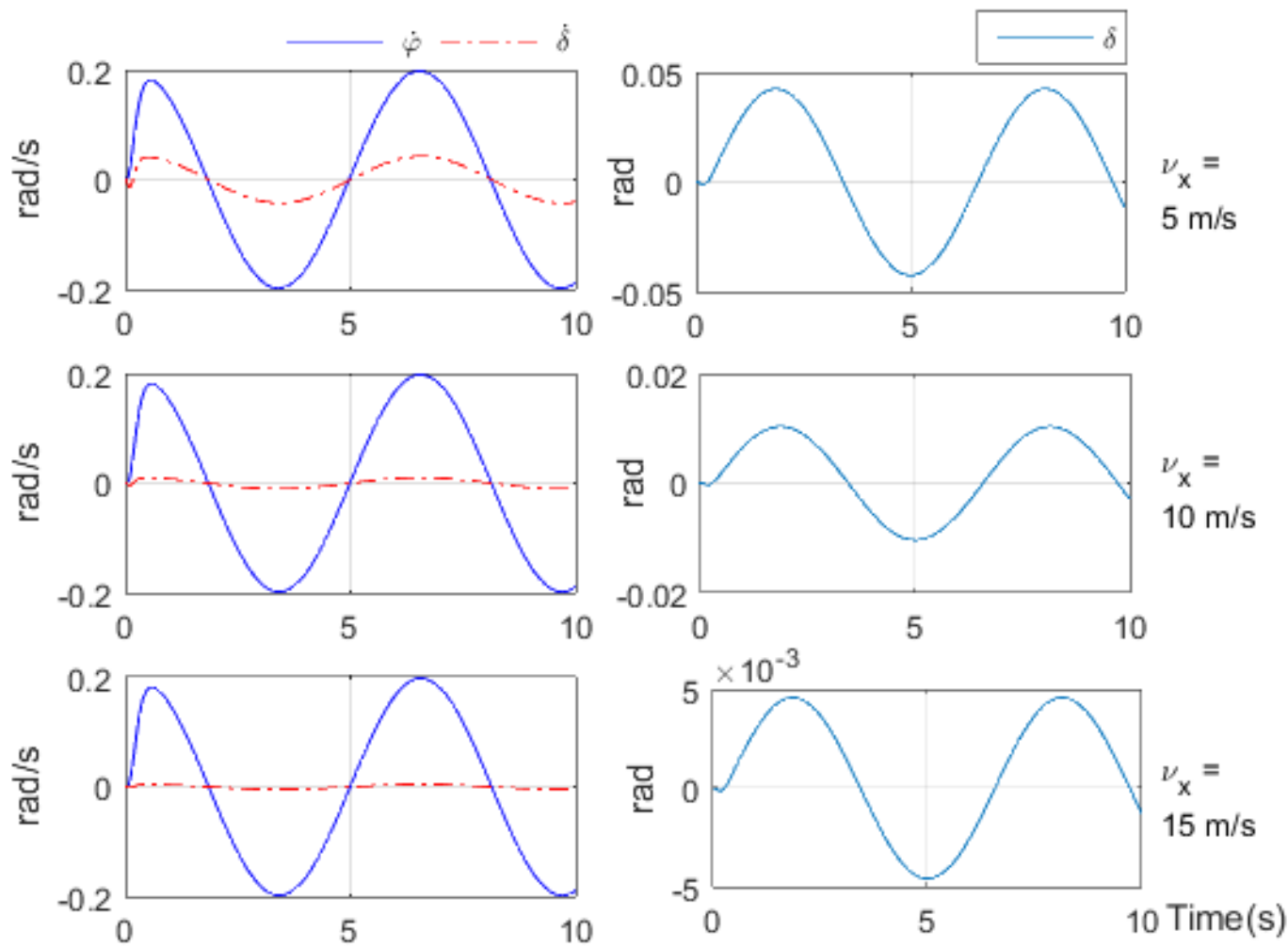

Figure 4.17 - Angular speed and position in simulation for roll control.

In order to analyze the efficiency of the proposed roll control, a comparison is made with the stability control via state feedback, presented in sub item 4.1. Using the same desired roll angle as an input - a sinusoidal wave with $0.2 \mathrm{rad}$ amplitude and $1 \mathrm{rad} / \mathrm{s}$ frequency -, the result for three different longitudinal speeds is shown in Figure 4.18.

The simulation demonstrates that, even though the state feedback strategy is capable of eliminating input noises on the roll angle and keeping it at a null steady state, it is not able to efficiently track a desired roll angle. In all three longitudinal speeds, the resulting roll angle has a sinusoidal pattern with the expected frequency still presenting a gain in comparison with the desired output. Therefore, the state feedback strategy is capable of stabilizing the system, but not of tracking a desired roll angle. Thus, the new roll angle control is necessary for the path preview strategy, in which a precise roll movement is crucial to the trajectory developed by the vehicle. 

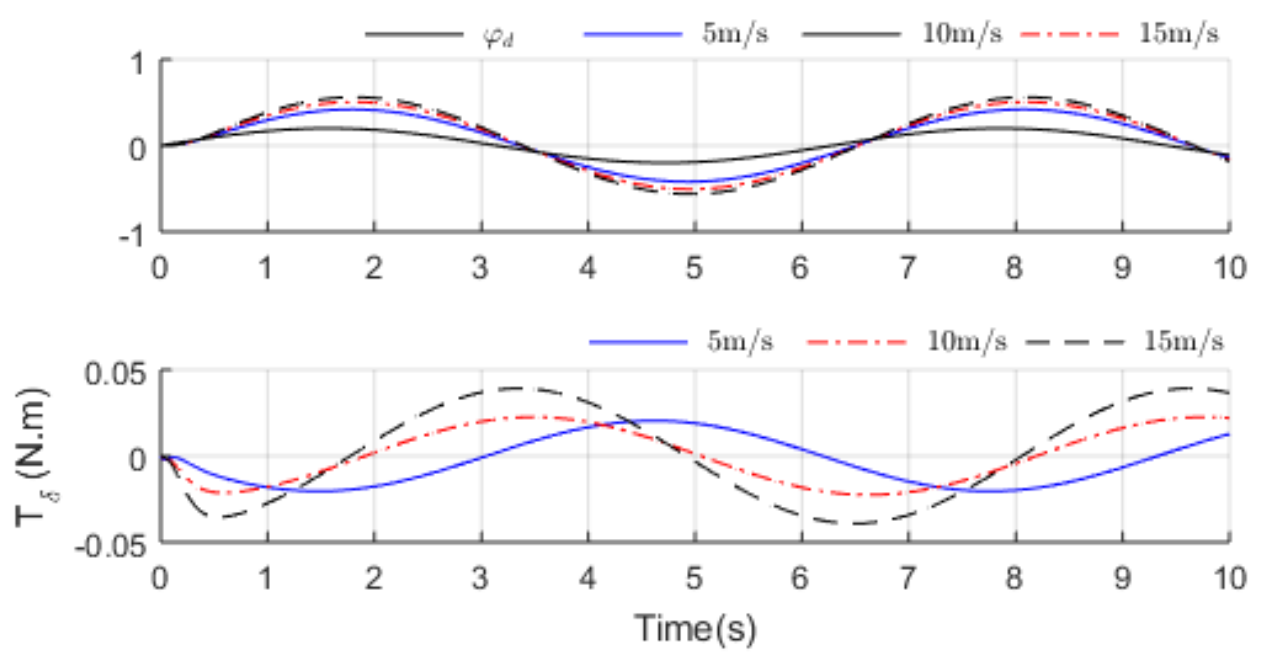

Figure 4.18 - Comparison between roll control strategies.

Once the motorcycle has an effective stabilization control, the next step is to guarantee its trajectory tracking ability. The main goal of this second loop is to minimize the distance error $e_{P}$ between the vehicle's current position and the predicted target path, illustrated in Figure 4.19.

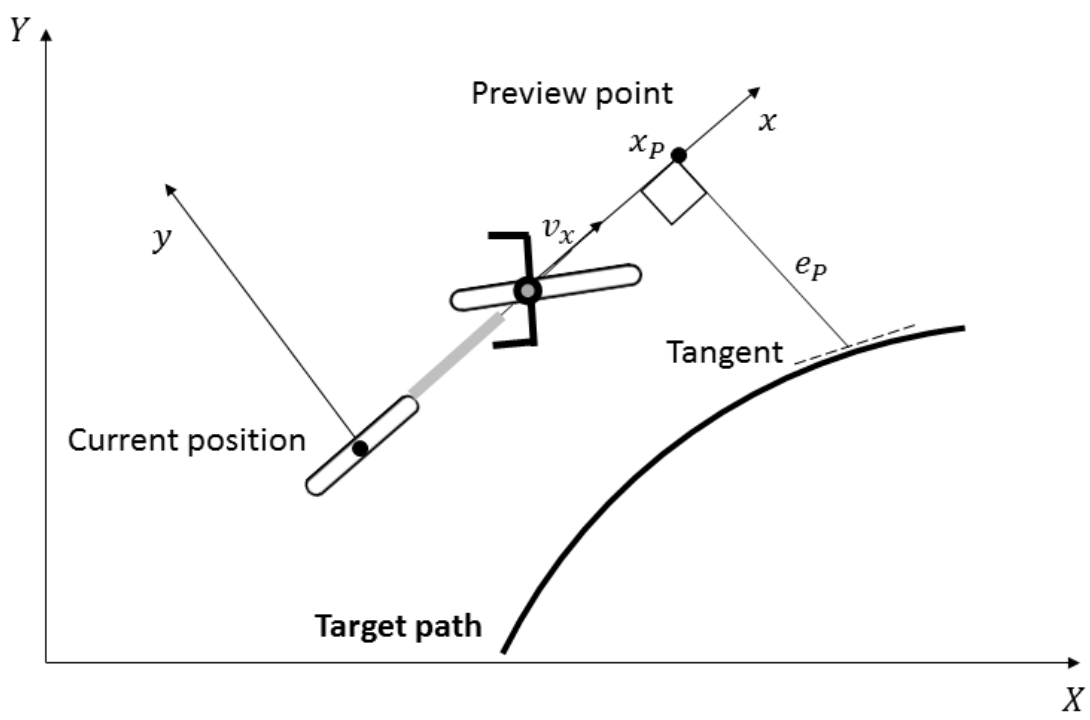

Figure 4.19 - Path preview schematic.

Human drivers look forward to a preview point on the same direction the vehicle is moving; the distance of this point is speed dependent, being closer to the current position the lower the velocity. Considering small yaw angles, the motorcycle is parallel to the global reference system and a simplification can be done to estimate the preview point given a time window (Eq. (4.22)). 


$$
x_{P}=x+v_{x} T_{P} \quad y_{p}=v_{x} T_{p} \psi
$$

With the preview point determined, it is possible to calculate the lookahead error $e_{P}$, which is the trajectory tracking error projected a distance in front of the vehicle, as described in Eq. (4.23). The trajectory error $\left(e_{p}\right)$ is the difference between the next estimated vehicle position in the global $Y$ axis and the next target lateral position - defined by a function $f$ of longitudinal distance. The trajectory error has a positive value when the target path is on the right side of the motorcycle and negative otherwise.

$e_{P}=y_{p}-f\left(x_{p}\right)$

With the distance and orientation error between the target path and the actual vehicle trajectory, it is possible to build the path-tracking controller as in Figure 4.20. The position error generate a reference roll angle, in order to keep the vehicle stable while pursuing the desired track.

Considering the countersteering effect, to make the motorcycle turn to one side, it is interesting to have a roll angular speed initially to the opposite side, creating a momentum, which will help the vehicle negotiate the curve. Therefore, when the motorcycle has to turn to the right - positive distance error $e_{p}$ between vehicle and target path - its body should tilt to the left, which represents a negative roll angle on the considered vehicle's coordinate system.

Thus, contrary to what is commonly done with feedback gains, the values of distance error gains are negative, to ensure a roll angular speed in the opposite direction of the motorcycle lateral displacement. These values were adjusted considering a longitudinal speed of $15 \mathrm{~m} / \mathrm{s}$ and a time preview of 0.5 seconds. The best results were obtained with a distance gain of $K_{P}=-0.7$ and an additional derivative gain was added to the displacement error to attenuate trajectory oscillations, with the value of $K_{d p}=-0.1$. 


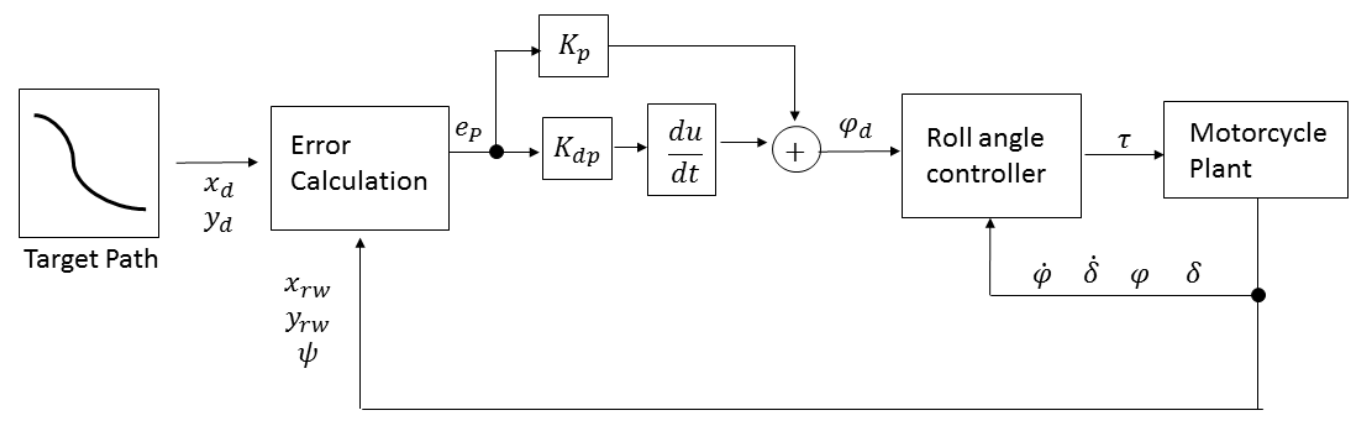

Figure 4.20 - Block diagram for trajectory control through path preview.

Finally, in order to validate the proposed control strategy, the same desired trajectory from previous simulations was used as input, a double lane change maneuver with lateral displacement of one meter.

Table 4.6 brings the error between desired and simulated roll angles and lateral trajectories to three different longitudinal speeds; the higher amplitude of the roll control angle was approximately $0.2 \mathrm{rad}$, to the $15 \mathrm{~m} / \mathrm{s}$ longitudinal speed. Though the roll control presents higher errors - in the magnitude of $40 \%$ of the total amplitude - the trajectory controller was able to keep the vehicle at under $4 \%$ error, which is a very effective performance.

Table 4.6 - Maximum error to path preview controller.

\begin{tabular}{lcccc}
\hline $\begin{array}{l}\text { Speed } \\
(\mathrm{m} / \mathrm{s})\end{array}$ & $\begin{array}{l}\text { Roll control } \\
\text { error }(\mathrm{rad})\end{array}$ & $\begin{array}{l}\text { Roll control } \\
\text { error }(\%)\end{array}$ & $\begin{array}{l}\text { Path tracking } \\
\text { error }(\mathrm{m})\end{array}$ & $\begin{array}{l}\text { Path tracking } \\
\text { error }(\%)\end{array}$ \\
\hline 5 & 0.055 & 39.3 & 0.010 & 1.0 \\
10 & 0.114 & 41.0 & 0.025 & 2.5 \\
15 & 0.177 & 42.1 & 0.039 & 3.9 \\
\hline
\end{tabular}

Figure 4.21 brings the trajectory behavior to the double lane change maneuver at three different longitudinal velocities; an overshoot is visible at high speeds, when the motorcycle has greater resistance to sudden trajectory changes. The torque applied to the handlebars (Figure 4.22) prove the motor saturation is satisfied. Figure 4.23 to Figure 4.25 shows the angular speeds and positions of the motorcycle and the countersteering is clear in the steer angle graphs, in which the handlebar is first turned to the opposite direction before being steered to the same direction of the trajectory. 


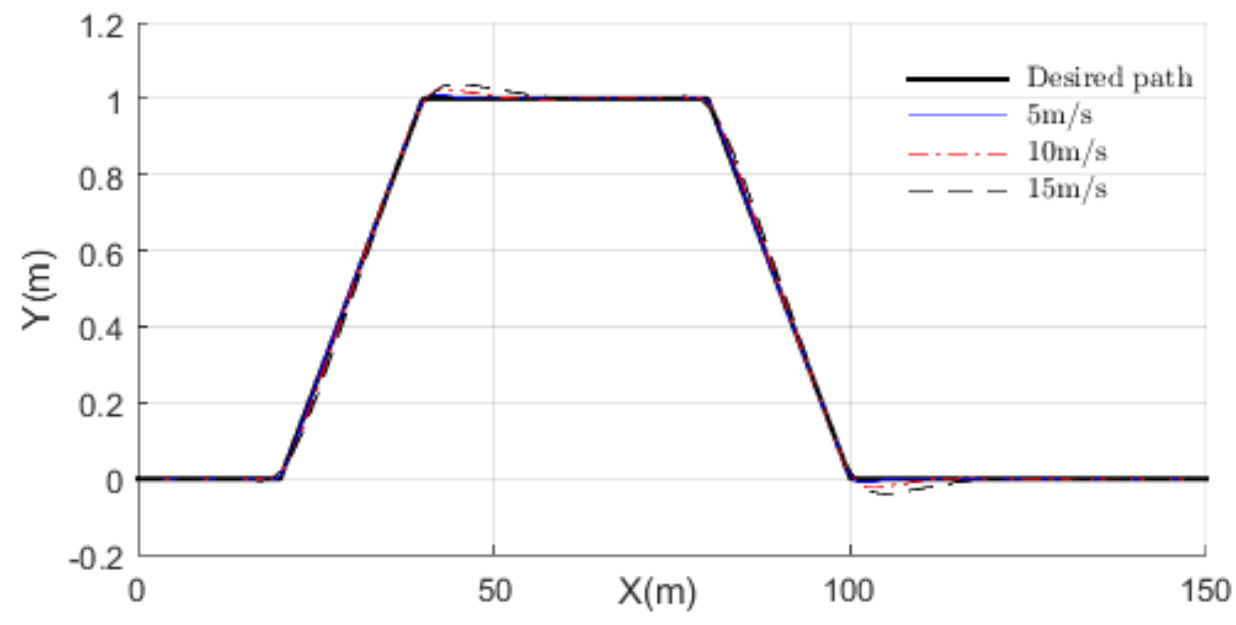

Figure 4.21 - Vehicle position and error in simulation for trajectory control through path preview.

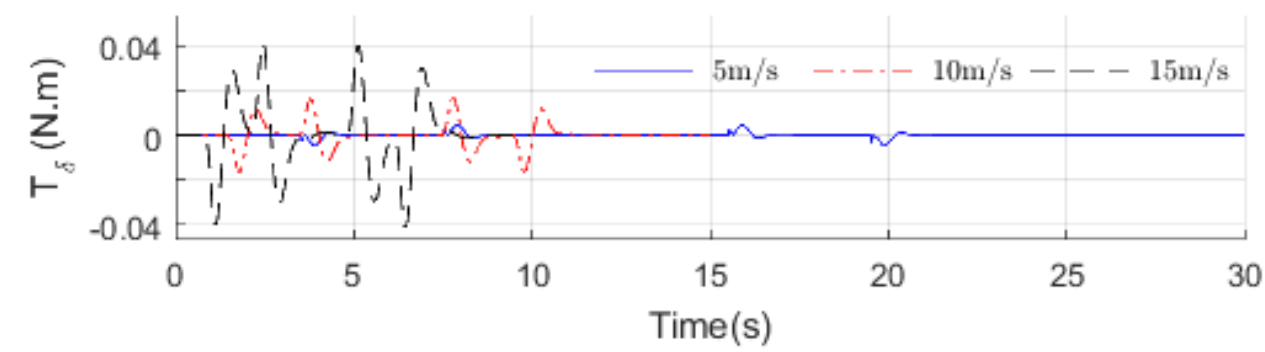

Figure 4.22 - Torque input for trajectory control through path preview.
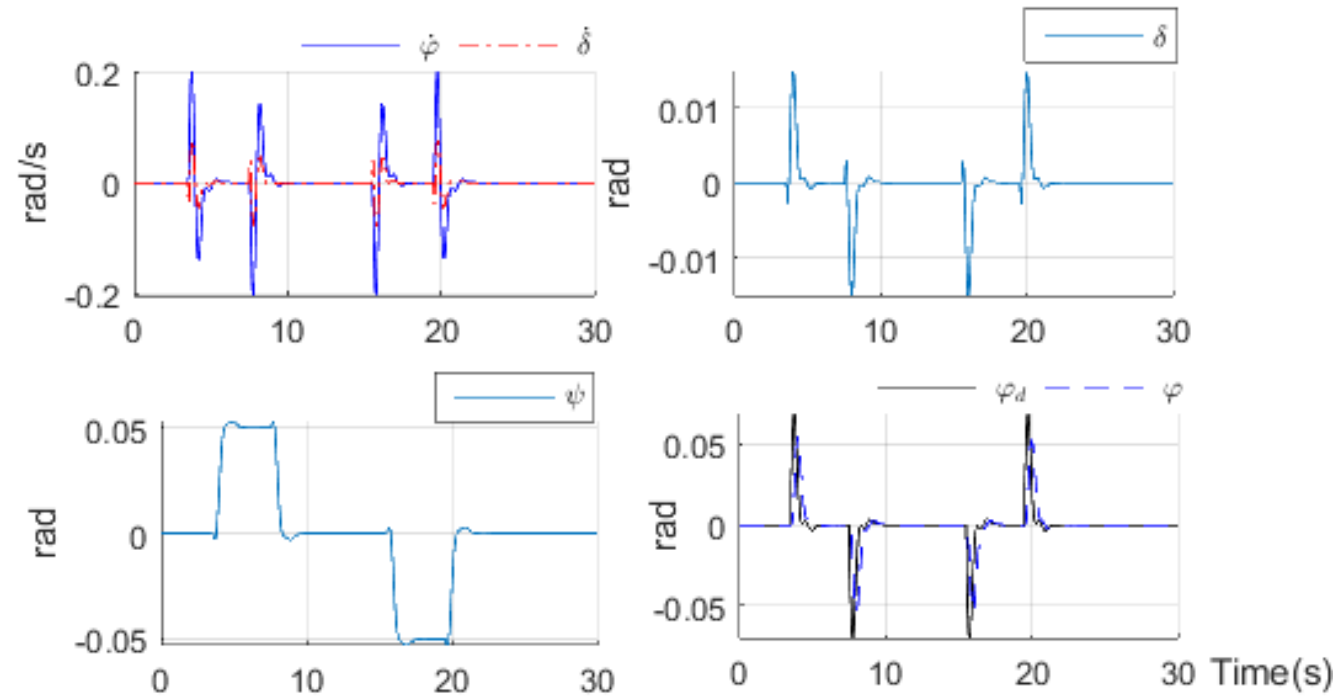

Figure 4.23 - Angular speed and position in simulation for trajectory control through path preview, at $5 \mathrm{~m} / \mathrm{s}$. 

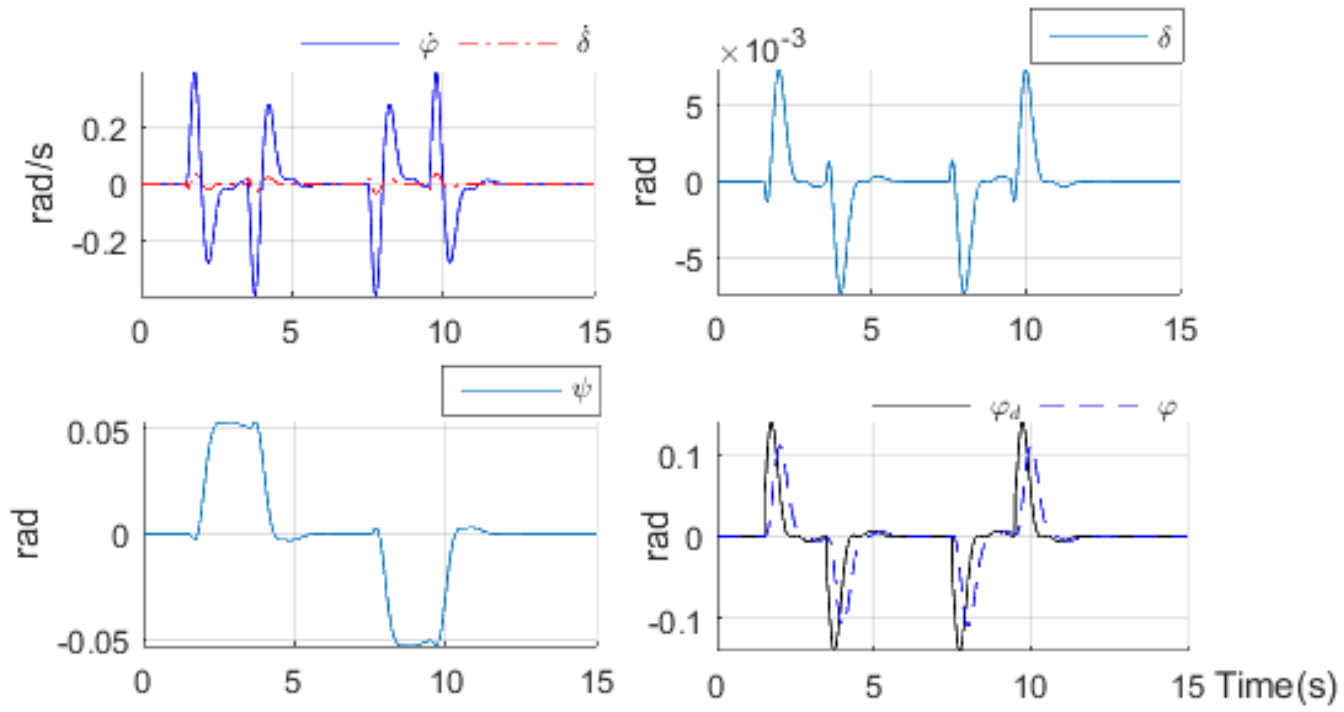

Figure 4.24 - Angular speed and position in simulation for trajectory control through path preview, at $10 \mathrm{~m} / \mathrm{s}$.
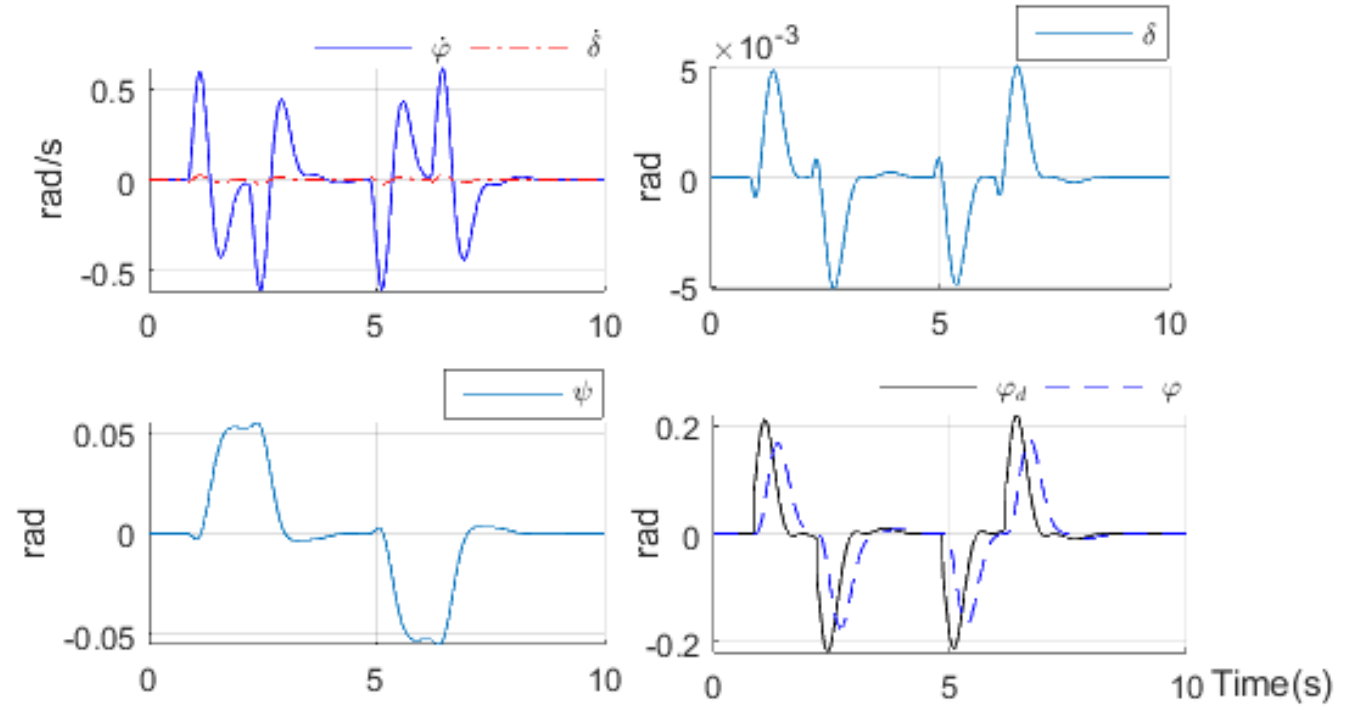

Figure 4.25 - Angular speed and position in simulation for trajectory control through path preview, at $15 \mathrm{~m} / \mathrm{s}$.

At last, it is interesting to compare the performance between all three trajectory control strategies: considering the speed of $10 \mathrm{~m} / \mathrm{s}$, Figure 4.26 brings the comparison between all three trajectory control strategies to the previous demonstrated double lane change maneuver.

An additional simulation was made, with the target path as the slalom maneuver, represented by a sine wave with two meters of amplitude and one $\mathrm{rad} / \mathrm{s}$ frequency. Figure 4.27 brings the final comparison between the full output feedback, linear quadratic regulator and path preview controllers. 
Both graphs illustrates how the output feedback controller and linear quadratic regulator present a similar performance, with a considerable delay between the target path and simulated trajectory, since the motorcycle steer and roll angles are actuated after it already exists an error between the vehicle's position and the desired road. The path preview controller presents an improved response due to its ability to react to the distance error before it actually happens.

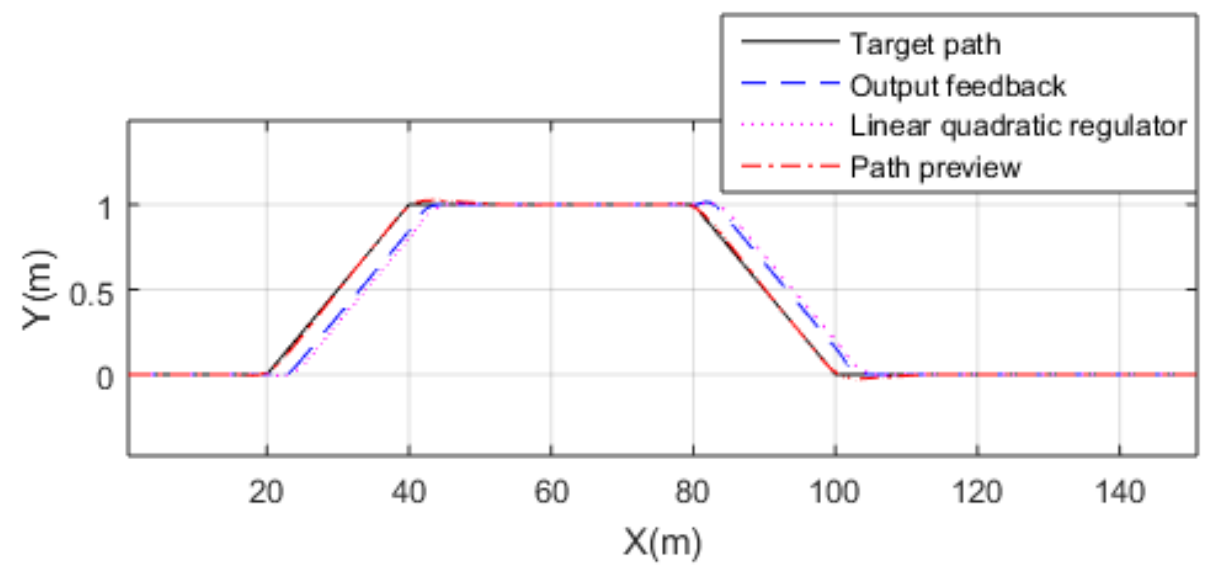

Figure 4.26 - Comparison between control strategies with the double lane change maneuver, at $10 \mathrm{~m} / \mathrm{s}$.

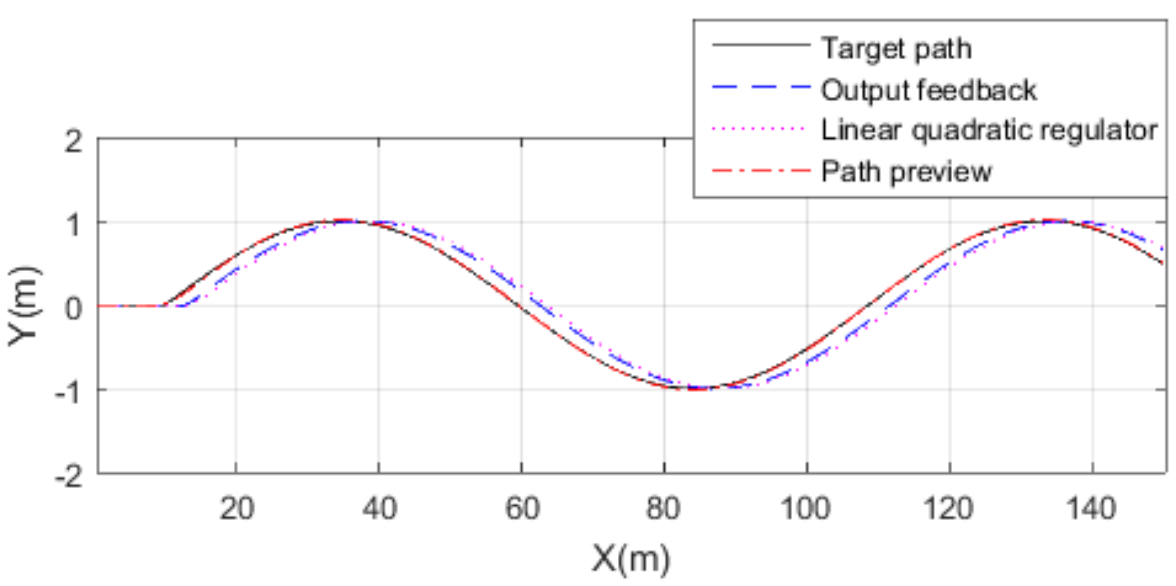

Figure 4.27 - Comparison between control strategies with the slalom maneuver, at $10 \mathrm{~m} / \mathrm{s}$. 
This chapter presented five control strategies, all based on modern control techniques such as pole placement and state observers. To guarantee the application of these procedures, it was first analyzed that the proposed dynamic model is completely observable and controllable, for every longitudinal speed considered.

Two control algorithms were aimed to solve the problem of natural vertical instability, present in two-wheeled vehicles and aggravated by the fact that the studied scale object does not display a self-stability speed range. The first controller assumed all state variables were ideally measured, while the second considered a state observer to estimate the variables not directly measured through sensors.

The following two control algorithms aimed to keep the motorcycle along a desired trajectory, through state feedback but different control gain vectors determination. The last control technique combines both objectives (stability and trajectory tracking) while decreasing the delay presented in the previous algorithms.

To apply any of the proposed control techniques to the experimental system, it is necessary to first analyze its embedded instrumentation. Since not all state variables are directly measured, due to high costs and physical barriers, the next chapter proposes a sensor fusion algorithm, in order to estimate the full state of the vehicle with the available sensors. 


\section{$5 \quad$ State estimation through sensor fusion technique}

An autonomous vehicle needs its instrumentation system to be embedded, in order to be completely independent in any environment - indoors or outdoors. To the studied object, because of its reduced scale, the size and weight of the instrumentation system is even more significant, since it should not influence the geometry and dynamic of the original motorcycle. Thus, an effective embedded instrumentation system has to be lightweight, reduced in size and with great reliability and performance.

The previous conditions indicate that redundant sensors must be eliminated, as it is less costly to use known relations between variables to estimate its values than to directly measure it. For this purpose, it is necessary an algorithm to effectively predict the unknown variables, based on their indirect relations and noisy measurements from sensors. One of the most outstanding algorithm in this task is the Kalman filter, in which the system's dynamical model, control inputs and multiple sequential measurements form an estimate of the system's state variables.

In this chapter, the Kalman filter is used to combine the noisy sensory data from four different sensors in the instrumentation system, as well as to estimate the state variables that are not directly measured from these devices. At last, this filtered and combined data can be used by the previously proposed control strategies as the measured state of the motorcycle.

\subsection{Kalman filter algorithm}

The Kalman filter is a mathematical algorithm that works in a two-step process: in the prediction step, it produces estimates of the current state variables based on a state-space model and the input to the system; in the update step, these estimates are adjusted using a weighted average between the predicted state and noisy measurements, with more weight being given to estimates with higher certainty. Since the filter uses not only the observable data but also the relation between state variables, it produces a better estimate than using individual sensor measurements. 
Considering a general continuous linear state-space model, as described by Eq. (5.1), an approximation of its discrete model is presented in Eq. (5.2), given a discretization period equal to $T$.

$$
\begin{aligned}
& \dot{X}=A X+B U \\
& Y=C X \\
& X_{k+1}=(I+T A) X_{k}+(T B) U_{k}=F_{k} X_{k}+G_{k} U_{k} \\
& Y_{k}=C X_{k}=H_{k} X_{k}
\end{aligned}
$$

The first step to the recursive Kalman filter is to provide the initial conditions, i.e., the estimated state variable $\left(\hat{x}_{k}\right)$ and estimated error covariance matrix $\left(\hat{P}_{k}\right)$ - which can be defined as the measure of the state estimate accuracy. If the state variable noises are considered independent, the matrix $\hat{P}_{k}$ can be simplified to a diagonal matrix.

The prediction step is then able to estimate the current variable state $\left(\bar{x}_{k}\right)$ and error covariance matrix $\left(\bar{P}_{k}\right)$ with the discrete model, control input and the previously estimated state and error covariance matrix (Eq. (5.3)). The matrix $Q$ is the covariance of the process noise, or the covariance of input $u_{k}$; a good estimate for this matrix is to consider the standard deviation of the input variables, which results in $Q=G_{k} G_{k}^{T} \sigma_{u}^{2}$.

$$
\begin{aligned}
& \bar{x}_{k}=F_{k} \hat{x}_{k}+G_{k} u_{k} \\
& \bar{P}_{k}=F_{k} \hat{P}_{k} F_{k}^{T}+Q
\end{aligned}
$$

An intermediate step is dedicated to calculate the optimal Kalman gain, Eq. (5.4), which is later used in the update step. The observation matrix $H$ defines which state variables are being measured by sensors while the observation noise covariance matrix $R$ models the noise variance from the devices measurements; a common estimate of this matrix is to consider the sensors noises independent, which results in $R=\operatorname{diag}\left(\sigma_{\text {sensor }_{i}}^{2}\right)$.

$$
K_{k}=\bar{P}_{k} H_{k}^{T}\left(H_{k} \bar{P}_{k} H_{k}^{T}+R\right)^{-1}
$$

On the update step, a new state $\left(\hat{x}_{k+1}\right)$ and error covariance $\left(\hat{P}_{k+1}\right)$ are estimated using the current predicted state and noisy measurements from the sensors $\left(z_{k}\right)$. The Kalman gain filter is then responsible for merging the 
information predicted from the discrete model with the data coming from the sensors, providing greater weight to whichever has less covariance, that is, relying more on the results with smaller error.

$\hat{x}_{k+1}=\bar{x}_{k}+K_{k}\left(z_{k}-H_{k} \bar{x}_{k}\right)$

$\hat{P}_{k+1}=\bar{P}_{k}-K_{k} H_{k} \bar{P}_{k}$

\subsection{Motorcycle sensor fusion strategy}

The motorcycle model used was the same applied to the path tracking strategies, reproduced in Eq. (5.6), considering only the steer torque as the control input of the system. The model has six state variables - roll and steer angular speeds and position; yaw angular velocity and lateral displacement and one additional element, the longitudinal speed, considered constant in this linear model.

$\left[\begin{array}{c}\ddot{\varphi} \\ \ddot{\delta} \\ \dot{\varphi} \\ \dot{\delta} \\ \dot{\psi} \\ \dot{y}_{r w}\end{array}\right]=\left[\begin{array}{cccccc}-0.48 v_{x} & -1.9 v_{x} & 75 & -14 v_{x}^{2}-13 & 0 & 0 \\ 2.7 v_{x} & -3.5 v_{x} & -6.6 & 0.33 v_{x}^{2}+144 & 0 & 0 \\ 1 & 0 & 0 & 0 & 0 & 0 \\ 0 & 1 & 0 & 0 & 0 & 0 \\ 0 & 0.09 & 0 & 1.76 v_{x} & 0 & 0 \\ 0 & 0 & 0 & 0 & v_{x} & 0\end{array}\right]\left[\begin{array}{c}\dot{\varphi} \\ \dot{\delta} \\ \varphi \\ \delta \\ \psi \\ y_{r w}\end{array}\right]+\left[\begin{array}{c}-100 \\ 566 \\ 0 \\ 0 \\ 0 \\ 0\end{array}\right]\left[T_{\delta}\right]$

For the sensor fusion algorithm, the instrumentation system needs to be related to the state variables described by the model. The sensors available in the work are: a three-axis accelerometer, which measures accelerations, in $\mathrm{m} / \mathrm{s}^{2}$; a three-axis gyrometer, which provides angular velocities, in rad/s; a tachometer, which gives the propulsion motor rotation, in rpm, and a potentiometer coupled to the handlebar, which measures the steering angle applied to the vehicle. Appendix $C$ illustrates the test bench used to verify the linear and angular accelerations measured by the IMU.

The inertial measurement unit (IMU) also contains a magnetometer, which measures the Earth's magnetic field and is usually used as a compass. This device was not used in this work because of strong magnetic influences from the electric motor, positioned very close to the sensor due to the reduced dimensions of the motorcycle.

Therefore, the motorcycle model variables directly measured by sensors are: the lateral acceleration determined by the accelerometer, roll and yaw angular speeds measured by the gyrometer, steer angle given by the 
potentiometer and the longitudinal speed, which can be inferred by the tachometer, considering no slippage at the rear wheel.

However, the measurements made by the IMU (accelerometer and gyrometer) cannot be considered directly equal to the motorcycle's acceleration and angular speeds because the device is not positioned at the considered coordinate origin point - at the contact point between rear wheel and the floor nor at the vehicle's center of mass. Thus, it is necessary to convert the sensors measurements to the correct origin point, which is done via a filter prior to the motorcycle dynamical model, based on Zhang et al (2013); additionally, the tachometer's data can be fused with the accelerometer to obtain a more reliable longitudinal speed value.

\subsubsection{IMU conversion filter}

Considering the motorcycle and the rear-frame fixed IMU, three main reference systems can be considered (Figure 5.1): the inertial frame, $l$, fixed to the ground; the plane $R$, moving with the motorcycle and with its $x$ axis aligned to the wheels' contact points with the floor - its origin is at the rear wheel (point $O)$ and it has a yaw angle $(\psi)$ in relation to the reference $I$. Finally, the plane $B$, fixed to the motorcycle's main body, presents a roll angle $(\varphi)$ in relation to the plane $R$.

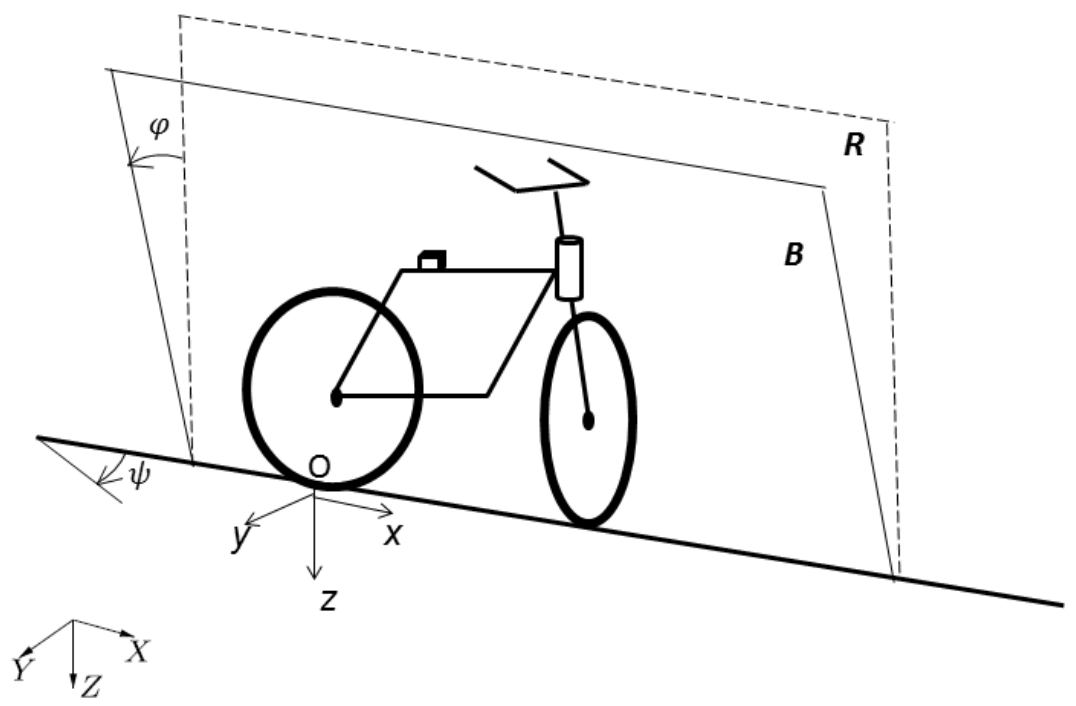

Figure 5.1 - Motorcycle rotational planes. 
The vehicle is considered to experience only two rotations: roll on the $x$ axis and yaw on the $z$ axis; this means the pitch angle, around $y$ axis, is disregarded as the motorcycle moves exclusively in flat terrain and, even with vibrations in its body, the IMU can be considered parallel to the ground.

The distance between the inertial measurement unit and the considered coordinate system's origin point $O$ is illustrated in Figure 5.2; the calculated values are $r_{x}=0.0986 \mathrm{~m}$ and $r_{z}=-0.2082 \mathrm{~m}$.

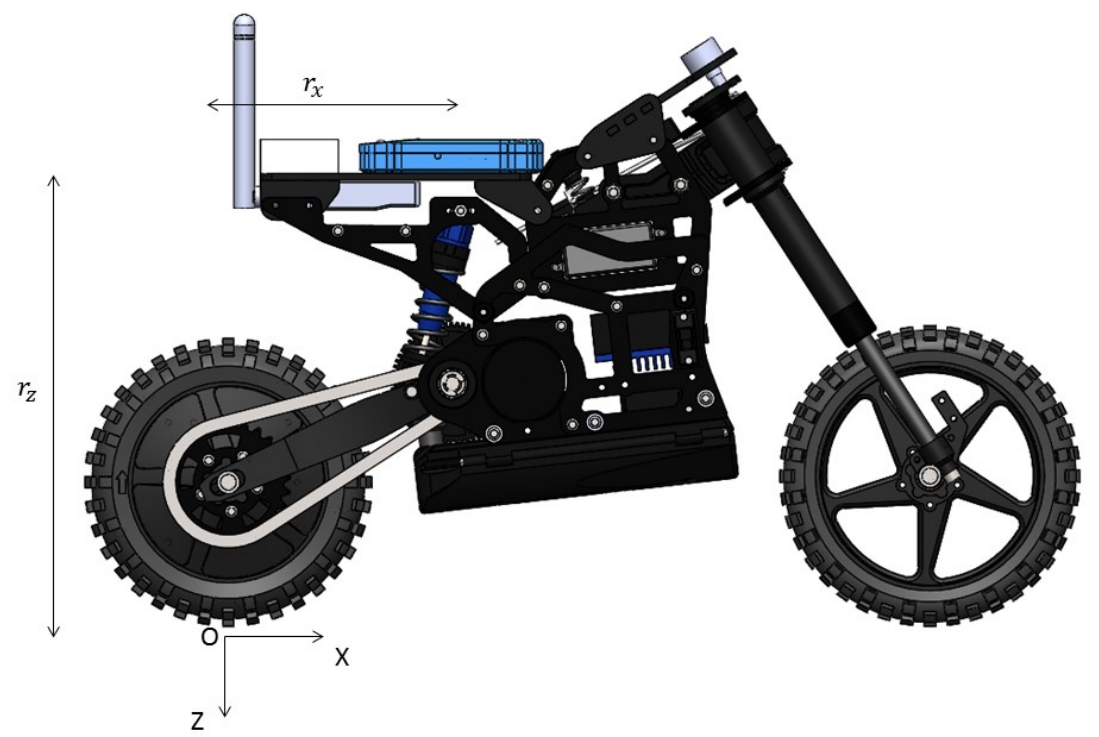

Figure 5.2 - Distance from IMU to origin point.

The motorcycle angular speeds at the origin point $O\left({ }^{R} \omega_{m}\right)$ can be correlated to the angular rates measured by the gyrometer $\left({ }^{B} \omega_{m}\right)$ by a rotation matrix. Considering ${ }_{B}^{R} R=R_{x}(\varphi)$ is the rotational matrix from $R$ to $B$, the inverse correlation is described in Eq. (5.7); the sine and cosine functions were simplified to, respectively, $s$ and $c$.

$$
\begin{aligned}
& { }^{B} \omega_{m}=R_{x}^{T}(\varphi){ }^{R} \omega_{m} \\
& {\left[\begin{array}{c}
\omega_{x} \\
\omega_{y} \\
\omega_{z}
\end{array}\right]=R_{x}^{T}(\varphi)\left[\begin{array}{l}
\dot{\varphi} \\
0 \\
\dot{\psi}
\end{array}\right]=\left[\begin{array}{c}
\dot{\varphi} \\
-s_{\varphi} \dot{\psi} \\
c_{\varphi} \dot{\psi}
\end{array}\right]}
\end{aligned}
$$


The direct relation between sensor and Euler angles is expressed in the previous equation; it is, however, the inverse relation that is used in the Kalman filter (Eq. (5.8)). There would be discontinuities in the model if the roll angle reaches 90 degrees, but this would be a situation of irreversible instability to the vehicle, and should be primarily avoided by the control algorithm.

$$
\left[\begin{array}{c}
\dot{\varphi} \\
\dot{\psi}
\end{array}\right]=\left[\begin{array}{ccc}
1 & 0 & 0 \\
0 & 0 & 1 / \cos (\varphi)
\end{array}\right]\left[\begin{array}{l}
\omega_{x} \\
\omega_{y} \\
\omega_{z}
\end{array}\right]
$$

The acceleration of origin point $O$ is described by Eq. (5.9), while Eq. (5.10) brings the angular rate of the motorcycle's body, both in the reference frame $R$. Eq. (5.11) illustrates the distance from the IMU to the origin point, considering the reference frame $B$.

$$
\begin{aligned}
& { }^{R} a_{O}=\left[\begin{array}{lll}
\ddot{x}_{r w} & \ddot{y}_{r w} & g
\end{array}\right]^{T} \\
& { }^{R} \omega_{m}=\left[\begin{array}{lll}
\dot{\varphi} & 0 & \dot{\psi}
\end{array}\right]^{T} \\
& { }^{B} r_{I M U}=\left[\begin{array}{lll}
r_{x} & 0 & -r_{z}
\end{array}\right]^{T}
\end{aligned}
$$

Thus, the acceleration of the IMU in the reference frame $R$ is calculated with the previous equations, resulting in Eq. (5.12). Eq. (5.13) brings the result to the same acceleration, but in the reference $B$, where the data is being acquired, i.e. ${ }^{B} a_{I M U}=\left[\begin{array}{lll}a_{x} & a_{y} & a_{z}\end{array}\right]^{T}$.

$$
\begin{aligned}
& { }^{R} a_{I M U}={ }^{R} a_{O}+{ }^{R} \dot{\omega}_{m} \times{ }_{B}^{R} R{ }^{B} r_{I M U}+{ }^{R} \omega_{m} \times{ }^{R} \omega_{m} \times{ }_{B}^{R} R{ }^{B} r_{I M U} \\
& { }^{R} a_{I M U}=\left[\begin{array}{c}
\ddot{x}_{r w} \\
\ddot{y}_{r w} \\
g
\end{array}\right]+\left[\begin{array}{ccc}
0 & 0 & -r_{z} s_{\varphi} \\
r_{z} c_{\varphi} & 0 & r_{x} \\
r_{z} s_{\varphi} & 0 & 0
\end{array}\right]\left[\begin{array}{c}
\ddot{\varphi} \\
0 \\
\ddot{\psi}
\end{array}\right]+\left[\begin{array}{c}
-\dot{\varphi} \dot{\psi} r_{z} c_{\varphi}-\dot{\psi}^{2} r_{x} \\
-\dot{\varphi}^{2} r_{z} s_{\varphi}-\dot{\psi}^{2} r_{z} s_{\varphi} \\
\dot{\varphi}^{2} r_{z} c_{\varphi}+\dot{\varphi} \dot{\psi} r_{x}
\end{array}\right] \\
& { }^{B} a_{I M U}={ }_{B}^{R} R^{T}{ }^{R} a_{I M U}=R_{x}^{T}(\varphi){ }^{R} a_{I M U}
\end{aligned}
$$

Finally, the acceleration of the motorcycle at the origin point $O$ can be calculated through the two previous equations; the result is in Eq. (5.14). Considering the vehicle's longitudinal speed is constant, its acceleration is null 
$(\ddot{x} \approx 0$ ); the linear acceleration parameters are described as nonlinear functions of the accelerometer and gyrometer measurements, Eq. (5.15).

$$
\begin{aligned}
& { }^{R} a_{O}=R_{x}(\varphi){ }^{B} a_{I M U}-{ }^{R} \dot{\omega}_{m} \times{ }_{B}^{R} R{ }^{B} r_{I M U}-{ }^{R} \omega_{m} \times{ }^{R} \omega_{m} \times{ }_{B}^{R} R{ }^{B} r_{I M U} \\
& {\left[\begin{array}{c}
\ddot{x}_{r w} \\
\ddot{y}_{r w} \\
g
\end{array}\right]=\left[\begin{array}{c}
a_{x} \\
a_{y} c_{\varphi}-a_{z} s_{\varphi} \\
a_{y} s_{\varphi}+a_{z} c_{\varphi}
\end{array}\right]-\left[\begin{array}{cc}
0 & -r_{z} s_{\varphi} \\
r_{z} c_{\varphi} & r_{x} \\
r_{z} s_{\varphi} & 0
\end{array}\right]\left[\begin{array}{c}
\ddot{\varphi} \\
\ddot{\psi}
\end{array}\right]-\left[\begin{array}{c}
-\dot{\varphi} \dot{\psi} r_{z} c_{\varphi}-\dot{\psi}^{2} r_{x} \\
-\dot{\varphi}^{2} r_{z} s_{\varphi}-\dot{\psi}^{2} r_{z} s_{\varphi} \\
\dot{\varphi}^{2} r_{z} c_{\varphi}+\dot{\varphi} \dot{\psi} r_{x}
\end{array}\right]} \\
& \ddot{\psi}=\frac{-1}{r_{z} s_{\varphi}}\left[a_{x}+\dot{\varphi} \dot{\psi} r_{z} c_{\varphi}+\dot{\psi}^{2} r_{x}\right] \\
& \ddot{\varphi}=\frac{1}{r_{z} s_{\varphi}}\left[a_{y} s_{\varphi}+a_{z} c_{\varphi}-g-\dot{\varphi}^{2} r_{z} c_{\varphi}-\dot{\varphi} \dot{\psi} r_{x}\right] \\
& \ddot{y}_{r w}=a_{y} c_{\varphi}-a_{z} s_{\varphi}-r_{z} c_{\varphi} \ddot{\varphi}-r_{x} \ddot{\psi}+\dot{\varphi}^{2} r_{z} s_{\varphi}+\dot{\psi}^{2} r_{z} s_{\varphi}
\end{aligned}
$$

The defined IMU conversion equations provide the motorcycle's lateral linear acceleration and roll and yaw angular speeds; however, the desired state variables to the dynamical motorcycle model are the longitudinal and lateral position, as well as roll and yaw angles. Thus, it is necessary to manipulate the IMU results to obtain the cited parameters.

Considering the linear and angular speeds and accelerations constant for small time intervals, Eq. (5.8) and (5.15) can be discretized through a kinematic model, presented in Eq. (5.16), where $a_{k}$ represents the system's acceleration, $v_{k}$ its velocity and $p_{k}$, position while $T$ is the discretization interval.

$$
\begin{aligned}
& v_{k+1}=v_{k}+a_{k} T \\
& p_{k+1}=p_{k}+v_{k} T+a_{k} T^{2} / 2
\end{aligned}
$$

The kinematic model to the IMU fusion data is explicit in Eq. ((5.17), considering all linear and angular parameters. The combined gyrometer and accelerometer data (Eq. (5.15)) become the input $u$ to the model, while the gyrometer data (Eq. (5.8)) is a measurement in the vector $z$.

The rear wheel tachometer also provides information on the longitudinal speed $v_{x}$ and, considering the small yaw angle hypothesis, the lateral speed is $v_{y}=v_{x} \psi$ (Eq. (3.13)). The roll and yaw angles will be obtained via the dynamical motorcycle model; Figure 5.3 illustrates the data flow on this kinematic Kalman filter. 


$$
\begin{aligned}
& {\left[\begin{array}{l}
x_{r w} \\
\dot{x}_{r w} \\
y_{r w} \\
\dot{y}_{r w} \\
\varphi \\
\dot{\varphi} \\
\psi \\
\dot{\psi}
\end{array}\right]_{k+1}\left[\begin{array}{llllllll}
1 & T & 0 & 0 & 0 & 0 & 0 & 0 \\
0 & 1 & 0 & 0 & 0 & 0 & 0 & 0 \\
0 & 0 & 1 & T & 0 & 0 & 0 & 0 \\
0 & 0 & 0 & 1 & 0 & 0 & 0 & 0 \\
0 & 0 & 0 & 0 & 1 & T & 0 & 0 \\
0 & 0 & 0 & 0 & 0 & 1 & 0 & 0 \\
0 & 0 & 0 & 0 & 0 & 0 & 1 & T \\
0 & 0 & 0 & 0 & 0 & 0 & 0 & 1
\end{array}\right]\left[\begin{array}{l}
x_{r w} \\
\dot{x}_{r w} \\
y_{r w} \\
\dot{y}_{r w} \\
\varphi \\
\dot{\varphi} \\
\psi \\
\dot{\psi}
\end{array}\right]+\left[\begin{array}{ccc}
0 & 0 & 0 \\
0 & 0 & 0 \\
T^{2} & & \\
\frac{2}{2} & 0 & 0 \\
T & 0 & 0 \\
0 & \frac{T^{2}}{2} & 0 \\
0 & T & 0 \\
0 & 0 & \frac{T^{2}}{2} \\
0 & 0 & T
\end{array}\right]\left[\begin{array}{l}
\ddot{y}_{r w} \\
\ddot{\varphi} \\
\ddot{\psi}
\end{array}\right]} \\
& z=\left[\begin{array}{l}
\text { Gyrometer } \\
\text { Gyrometer } \\
\text { Tachometer } \\
\text { Tachometer } * \psi
\end{array}\right]=\left[\begin{array}{llllllll}
0 & 0 & 0 & 0 & 0 & 1 & 0 & 0 \\
0 & 0 & 0 & 0 & 0 & 0 & 0 & 1 \\
0 & 1 & 0 & 0 & 0 & 0 & 0 & 0 \\
0 & 0 & 0 & 1 & 0 & 0 & 0 & 0
\end{array}\right]\left[\begin{array}{c}
x_{r w} \\
\dot{x}_{r w} \\
y_{r w} \\
\dot{y}_{r w} \\
\varphi \\
\dot{\varphi} \\
\psi \\
\dot{\psi}
\end{array}\right]_{k}
\end{aligned}
$$

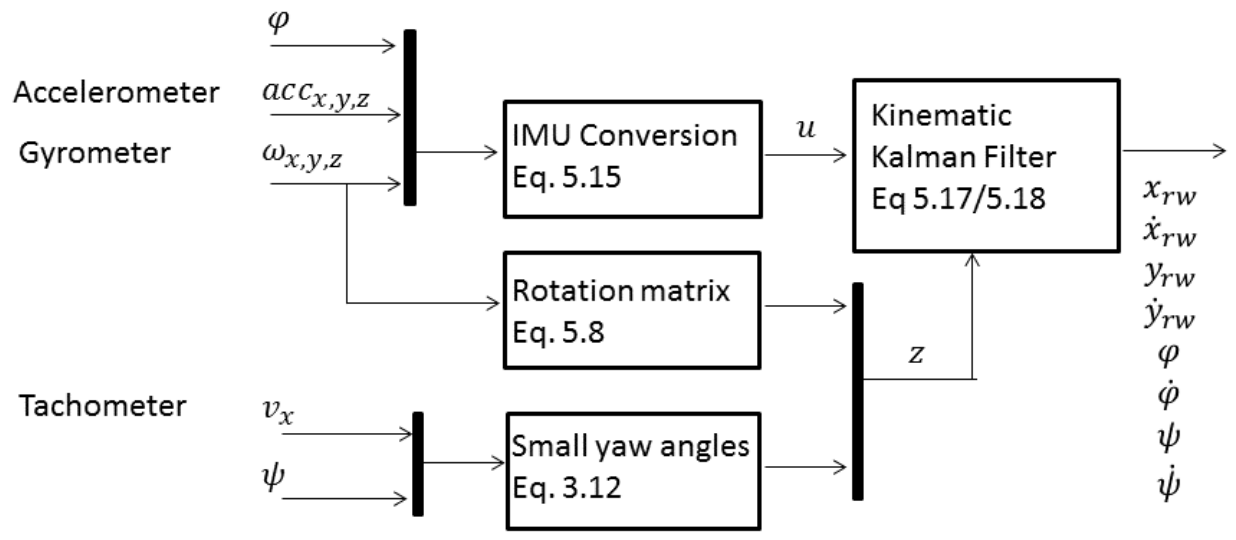

Figure 5.3 - Kinematic Kalman filter schematic.

Once the IMU data has been adjusted to the desired motorcycle state variables, it is possible to use the dynamical model to estimate the vehicle position. The kinematic filter provides the longitudinal velocity, used to build the continuous model, which is then discretized in the next step and the input torque $T_{\delta}$ is given by the control strategy. The potentiometer yields the steer angle at the handlebar, which is concatenate with the kinematic filter estimated variables to form the measurements vector (Eq. (5.19)); Figure 5.4 illustrates this process. 
$z=\left[\begin{array}{l}\text { Kinematic filter } \\ \text { Kinematic filter } \\ \text { Potentiometer } \\ \text { Kinematic filter } \\ \text { Kinematic filter }\end{array}\right]=\left[\begin{array}{cccccc}1 & 0 & 0 & 0 & 0 & 0 \\ 0 & 0 & 1 & 0 & 0 & 0 \\ 0 & 0 & 0 & 1 & 0 & 0 \\ 0 & 0 & 0 & 0 & 1 & 0 \\ 0 & 0 & 0 & 0 & 0 & 1\end{array}\right]\left[\begin{array}{c}\dot{\varphi} \\ \dot{\delta} \\ \varphi \\ \delta \\ \psi \\ y_{r w}\end{array}\right]_{k}$

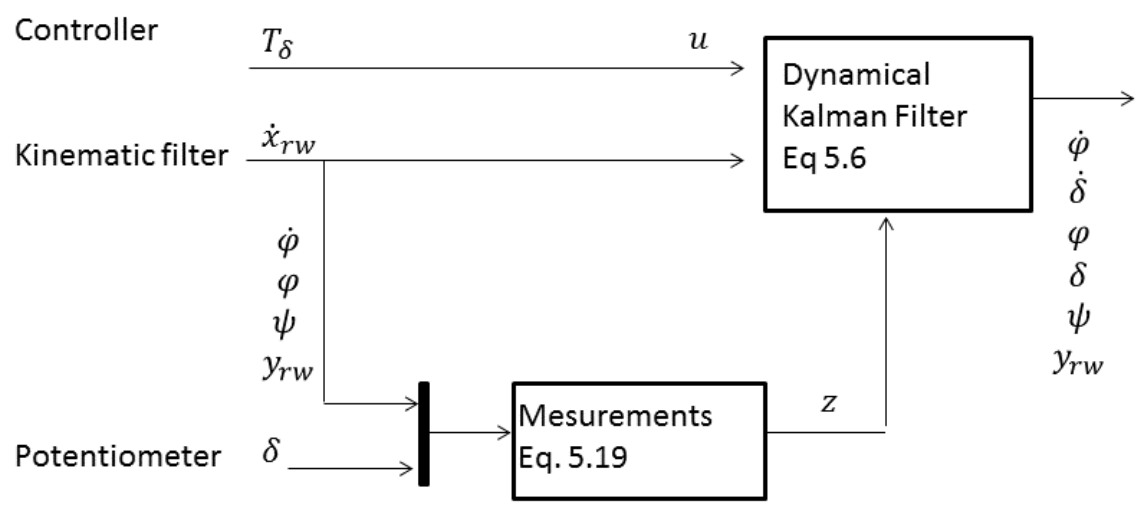

Figure 5.4 - Dynamical filter schematic.

To obtain the final motorcycle state, the kinematic and dynamical filters are concatenated (Figure 5.5). First, the noisy measurements from accelerometer, gyrometer and tachometer are fused and filtered by the IMU conversion filter, which provides the motorcycle's longitudinal speed, lateral position, roll angular rate and position and yaw angle. Then, this output is used as measured data by the dynamical filter, with the addition of the potentiometer's measurements and the steer torque input, given by the control algorithm. The output from the second filter is the vehicle's full state: the yaw angle information is fed back to the kinematic filter while the full state is used as input by the controller, resulting in the steer torque, closing the loop.

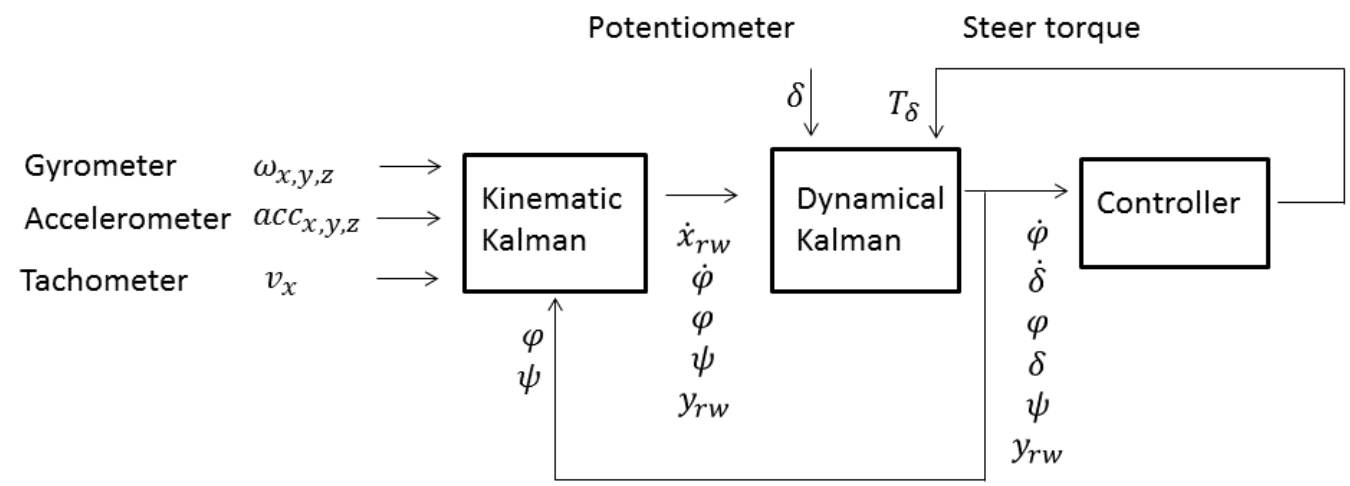

Figure 5.5 - Concatenated Kalman filters schematic. 


\subsubsection{Results}

In order to verify the validity of the proposed sensor fusion filter, the path preview simulation presented on the previous chapter was reproduced, with the double lane change and slalom maneuvers at three different speeds $(5,10$ and $15 \mathrm{~m} / \mathrm{s})$. The gyrometer data was generated using the relation explicit in Eq. (5.7), while the accelerometer measurements were fabricated via Eq. (5.14); the torque input was the same as the foreseen by the control algorithm while the tachometer and potentiometer data were assumed as the longitudinal speed and steer angle simulation results with white noise. The sampling interval was 0.05 second and Figure 5.6 illustrates a generated set of sensors data for the double lane change maneuver at $15 \mathrm{~m} / \mathrm{s}$.
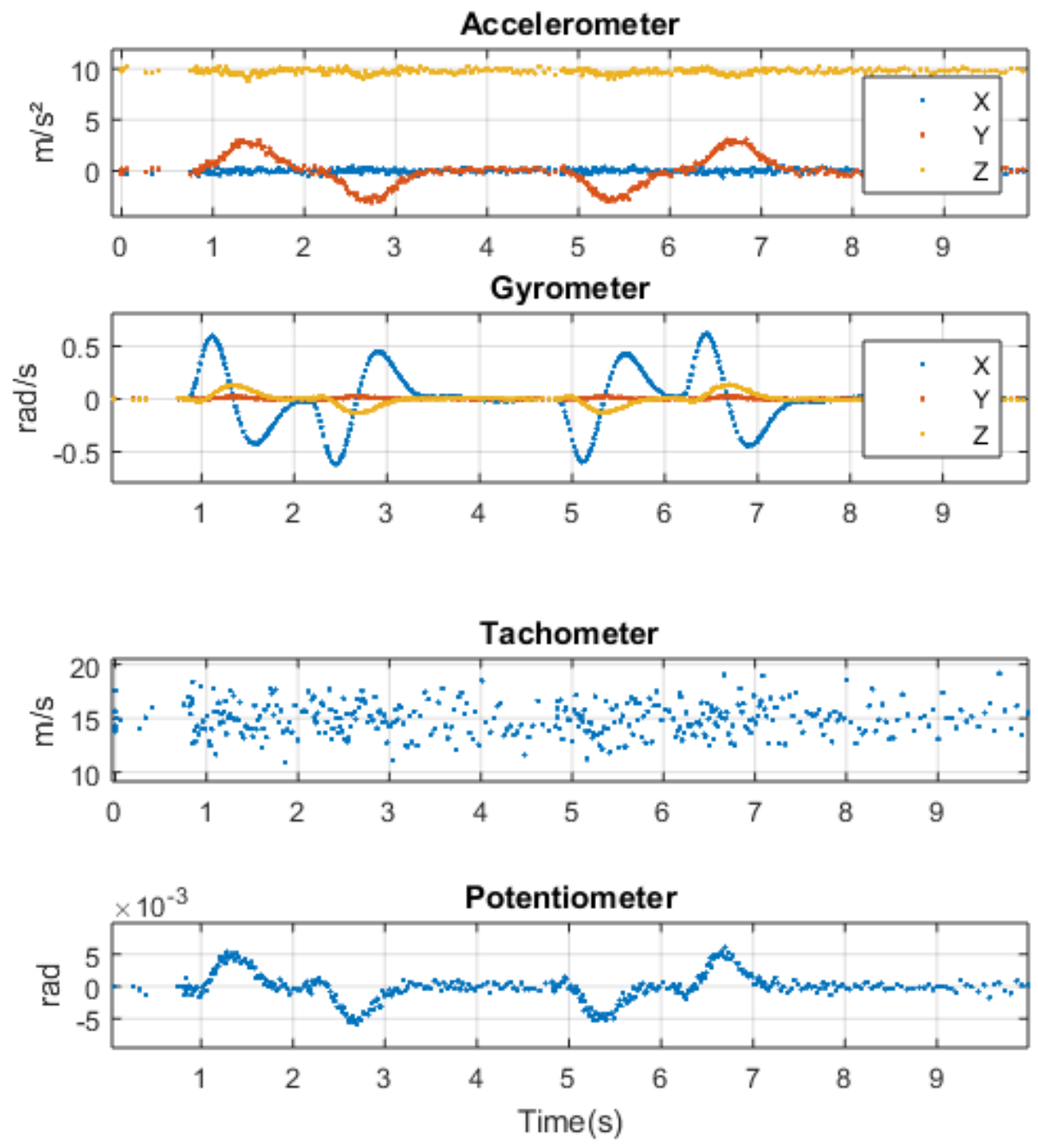

Figure 5.6 - Generated noisy data. 
The initial estimated state variable $\left(\hat{x}_{k}\right)$ was considered null on both filters, as well as the initial estimated error covariance matrix $\left(\hat{P}_{k}\right)$; all variable errors were considered independent, i.e. the process covariance matrix $Q$ and the state covariance matrix $R$ are diagonal matrices. All matrices on both filters were considered equal to the identity.

Figure 5.7 compares the filter performance for three different speeds by reproducing the double lane change maneuver, while Figure 5.8 does the same to the slalom trajectory. Though the filter is able to maintain the overall shape of the target path, a drift phenomenon is clear on both simulations; since the kinematic filter is an integration over time, this result was expected, due to error accumulation.

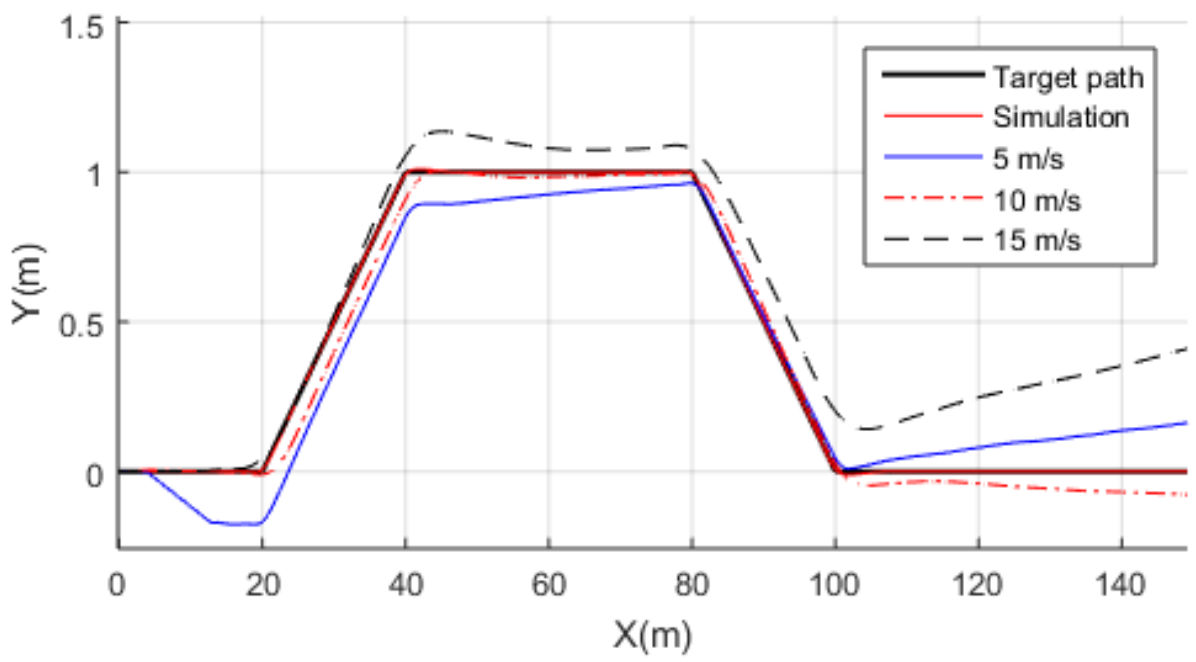

Figure 5.7 - Kalman filter on the double lane change maneuver.

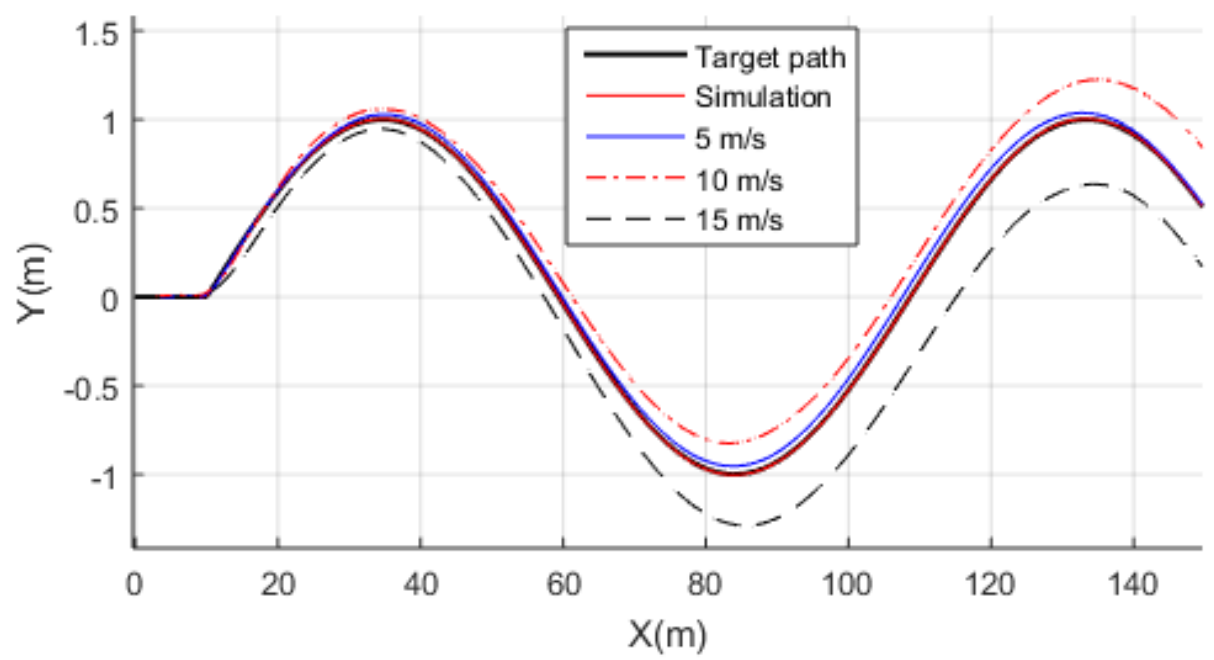

Figure 5.8 - Kalman filter on the slalom maneuver. 
This chapter presented a sensor fusion strategy to estimate the state variables of the dynamical model based on the embedded instrumentation system. The chosen approach was the Kalman filter, an algorithm that estimates the unknown state variables by combining noisy measurements and the expected result based on the state model.

The instrumentation system consisted of a tachometer, measuring the traction motor rotation; a potentiometer, sensing the steering angle, and an Inertial Measurement Unit (IMU), providing angular velocities through a gyrometer and linear accelerations through an accelerometer.

The first step for the sensor fusion was to calibrate all sensors; the tachometer and potentiometer were characterized in Chapter 2, while the IMU is analyzed in Appendix C. An additional step is necessary to correlate the IMU measurements to the state variables, since the sensor is positioned at a different location than the considered system origin. The sensor and the origin are considered as two points connect via a rigid body, with relative rotation and conjoined translation.

The IMU equations provide the motorcycle lateral and linear accelerations and roll and yaw angular speeds, which need to be integrated to become the considered state variables - longitudinal and lateral position, as well as roll and yaw angles.

The previous result is then combined with the potentiometer readings and torque input calculated from the control algorithm as input data to the Kalman filter, using the dynamic model of the motorcycle as a prediction state.

This concatenated double Kalman filter was tested on the double lane change simulations, considering the path preview control strategy, with the IMU measurements being simulated via an added white noise to the state variables. The estimated position maintained the overall shape of the target path, but presented an error accumulation over time - attributed to the considered integrations - which could be improved with lateral dynamic equations, instead of the considered kinematic relations.

With the system dynamic model, control strategy and embedded instrumentation characterized and combined, the next step is to test this complete system. The following chapter details an actuated platform capable of imitating the motorcycle's main dynamical behaviors, in order to act as a riskfree test bench for the proposed control strategies. 


\section{$6 \quad$ Actuated platform for motorcycle's simulation}

On the previous chapters, the mathematical model of the motorcycle dynamical behavior was developed; control algorithms to keep the vehicle stable and at a certain target path were designed and a filter to merge the data from different sensors was elaborated. Since, virtually, all the proposed algorithms were effective, the last step is to verify the feasibility and accuracy of these methods in the physical small scale system.

The obvious alternative would be to test the system in an open field, with the risk of it capsizing and even being permanent damaged should the control system not be properly adjusted. Thus, another strategy is presented in this chapter: an actuated platform to simulate the dynamical characteristics of a motorcycle, on a risk-free and student friendly environment.

\subsection{Hardware in the loop}

Simulators have the ability of reproducing real systems behaviors under safe and controlled circumstances; however, a reliable simulator can become costly and highly complex. On the other hand, computational models, alone, are not capable of predicting every response of the system to the real world. The hardware in the loop technique, then, appears as an intermediate solution between both approaches.

The latter technique consists of including some of the studied hardware in the computational simulation loop, rather than testing the algorithm performance in a purely numeric model. It has the main advantage of avoiding the need to replicate the full physical system while adding complexity and realism to the simulator.

A hardware-in-the-loop simulation data flow usually starts at an electrical emulation of the system sensors or actuators, which is controlled by the plant simulation and read by the embedded system under test. The former implements its control algorithm, changing the sensors or actuators signals, resulting in different variable values in the plant simulation, closing the feedback loop. Briefly, the hardware in the loop simulation interacts real sensors with simulated actuators and plants. 
The proposed actuated platform is a hardware in the loop simulation since it uses the linear dynamical model of the motorcycle to determine the roll, yaw and lateral position of the vehicle, applies it to the physical system through actuators and reads the real-time response of its embedded instrumentation.

\subsection{Actuated platform}

The built actuated platform is able to imitate the motorcycle behavior on the plane due to three main degrees of freedom: the roll movement, yaw motion and lateral displacement. A fourth degree of freedom could be beneficial to the simulations, though not applied in this first prototype: the longitudinal movement, represented by a treadmill.

Figure 6.1 brings the SolidWorks model, which guided the dimensions of the project so that it would be light enough to use in demonstrations at engineering classes while also being visually interesting. The final object is represented in Figure 6.2, with the studied motorcycle attached.

The chosen degrees of freedom allow for the visualization of the motorcycle movements while its sensors process the accelerations and angular rates imposed to the system. The roll and yaw motions are executed by servomotors, whose position control ensure a higher angle accuracy; the first motion has a total range of thirty degrees while the second, of eighty degrees, which is sufficient to reproduce the considered linear movements of a twowheeled vehicle.

The lateral displacement, on the other hand, is implemented with an electric motor along with an encoder, which ratifies the travelled distance and ensures the platform's lateral position. The motor is directly attached to a gear connected to a strap, i.e., there is no reduction system. With 2064 steps at each motor revolution, connected to a gear of $0.675 \mathrm{~cm}$ radius, the precision of the system is of $0.0021 \mathrm{~cm} / \mathrm{step}$. The total displacement is of $10 \mathrm{~cm}$ : the initial motorcycle position is at the center of the track and, for safety reasons, the maximum course is limited to $4 \mathrm{~cm}$ to each side. Additionally, the maximum speed of the system, with the motorcycle attached, is of $0.015 \mathrm{~m} / \mathrm{s}$.

All three electric motors are actuated by an Arduino microcontroller, which is also responsible for the startup routine - guaranteeing the initial platform position, with the roll angle perpendicular to the ground, the yaw angle aligned with the $X$ axis and the lateral position at the center of the track - and shutdown routine, which reproduces the initial configuration. 


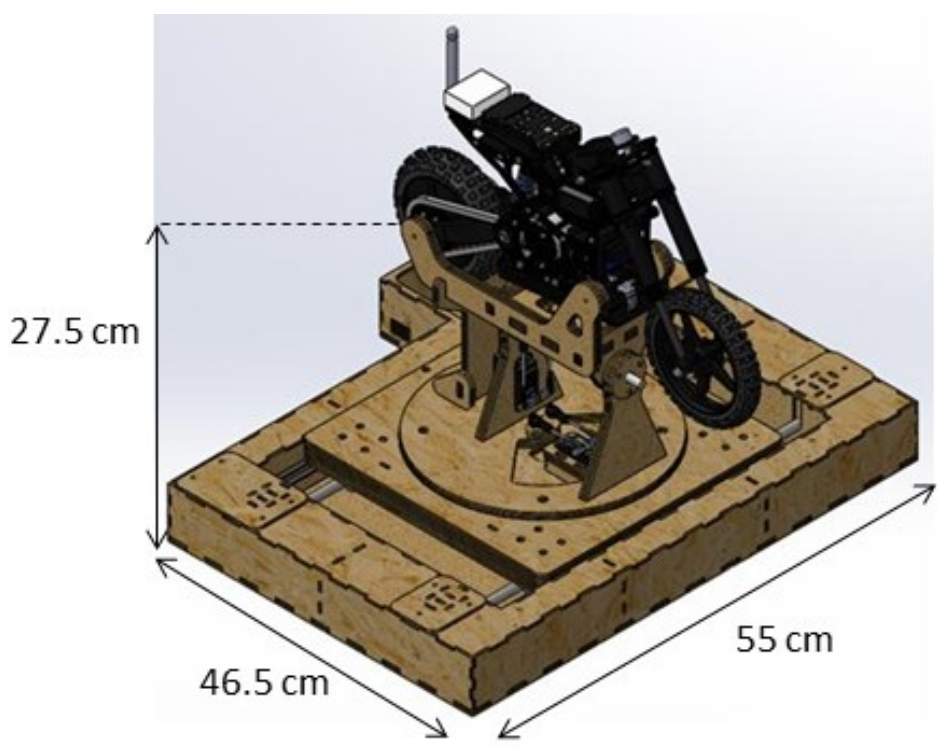

Figure 6.1 - Actuated platform concept.

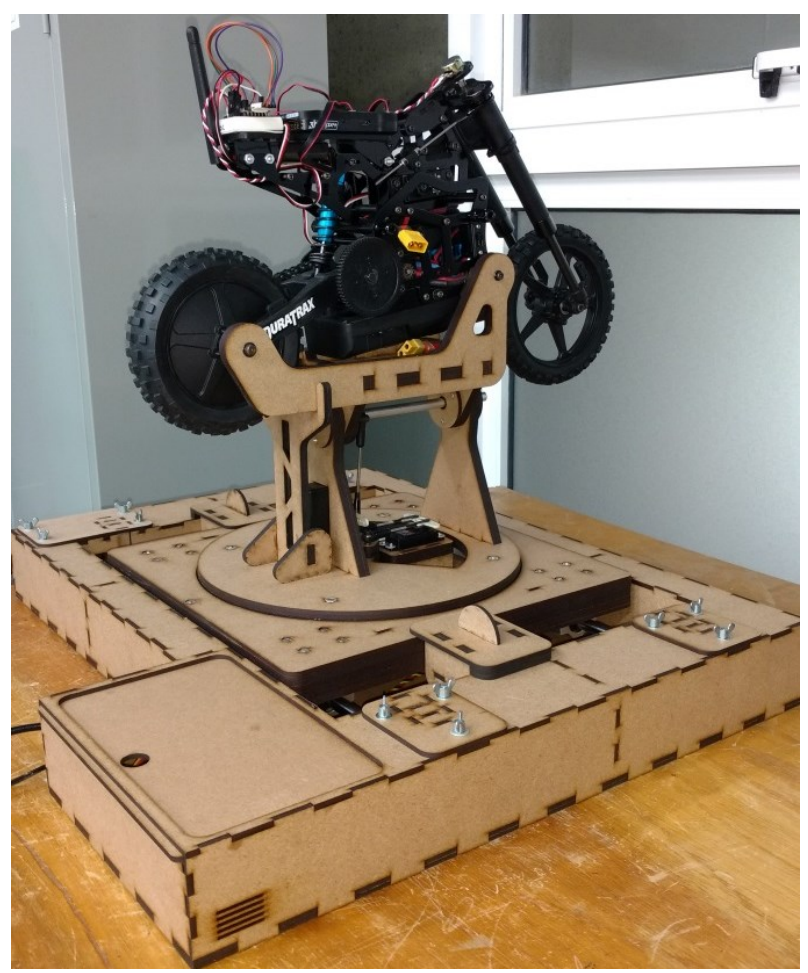

Figure 6.2 - Actuated platform device.

As for the software, a Labview program is the main responsible for commanding and integrating the multiple hardware on this simulator. In this first prototype, the program reads a Matlab simulation with the linear dynamical model of a motorcycle to determine the desired roll and yaw angle as well as lateral position; this information is sent to the Arduino board via serial communication, which then guarantees the three electrical motors reproduce the target behavior. 
Simultaneously, the Pixhawk controller embedded to the motorcycle is responsible for reading sensors data, filtering it and applying control techniques as if the vehicle was free to roll on the plane. All this information is, fed back to the computer via radio communication and received by the main Labview program. Figure 6.3 illustrates the data flow of the system.

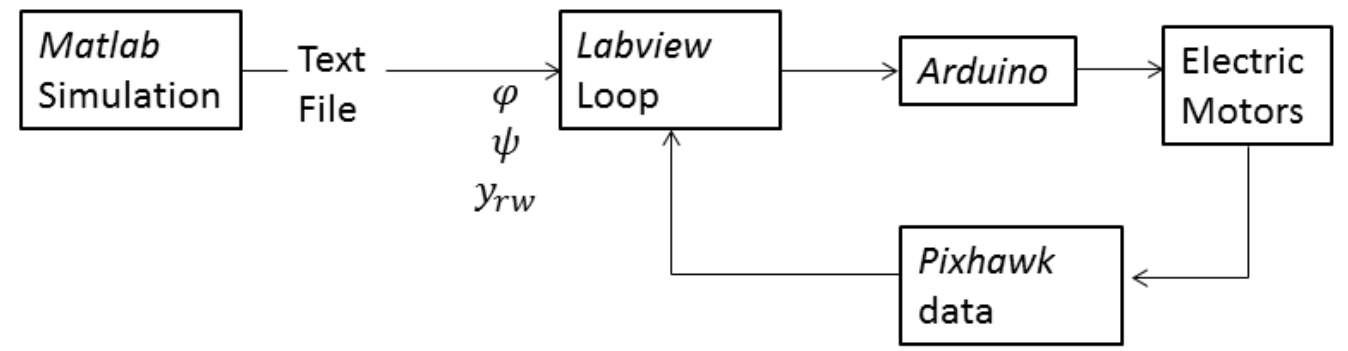

Figure 6.3 - Data flow on actuated platform.

The last step of this hardware in the loop simulator is to exhibit in real time a virtual representation of the motorcycle movement to the user. The final result is illustrated Figure 6.4: a rear view of the motorcycle is at the far right, followed by a $X-Z$ view of the motion (or exclusively the roll movement) and a X-Y view (a combined yaw and lateral displacement motion); the graphs indicate the executed trajectory as sensed by the embedded instrumentation.

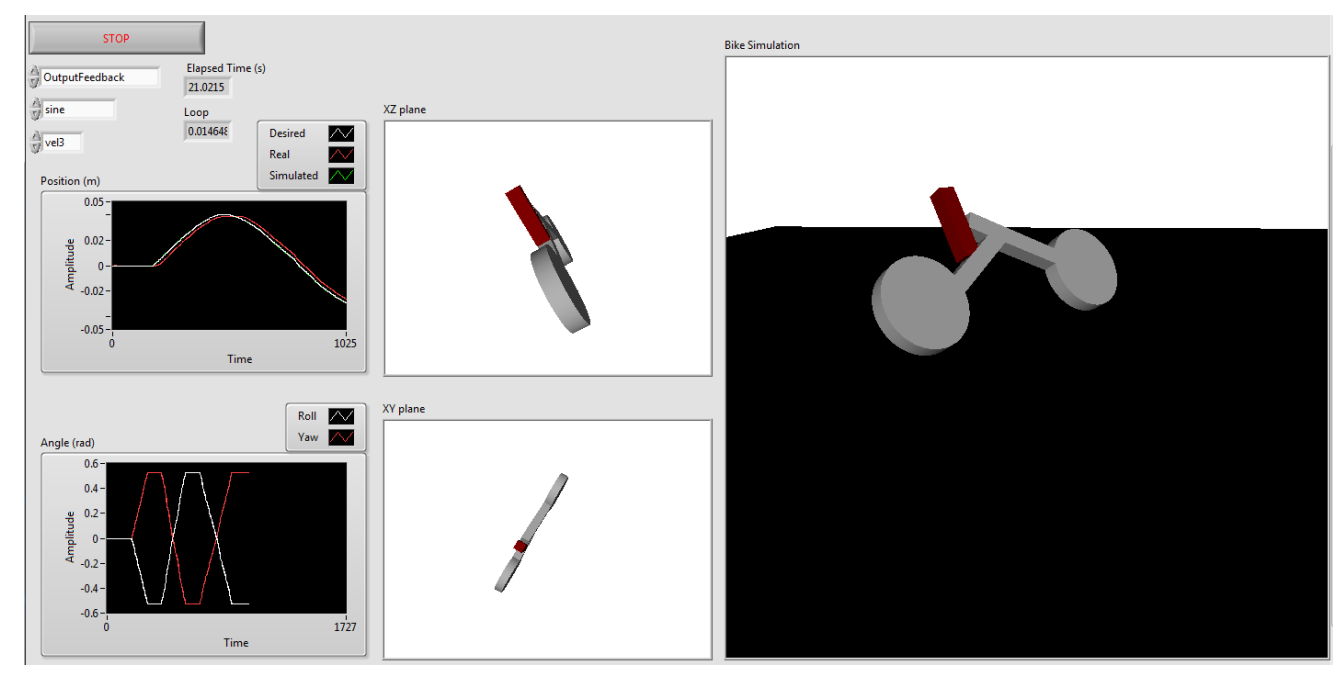

Figure 6.4 - Real-time Labview interface. 


\subsection{Experiments}

To test the platform, two experiments were performed: the first, intended to verify exclusively the roll and yaw motions, was a reproduction of the roll control simulation calculated in item 4.5 , for the longitudinal speed of $5 \mathrm{~m} / \mathrm{s}$. The desired roll angle is a sinusoidal function of 0.2 rad amplitude - or approximately 11.5 degrees - and the expected yaw angle is determined through its kinematic relation to the steer angular speed and position, described in Eq. (3.6). The result was a sinusoidal function of 0.7 rad amplitude (40 degrees), close to the maximum yaw movement physically allowed by the platform. The lateral displacement for this movement had larger amplitude than the platform track and was therefore disregarded.

Using the relation established on Eq. (5.8) and Eq. (5.15), it is possible to calculate the roll and yaw angles based on the gyrometer and accelerometer data. The measurements are in Figure 6.5 while the Kalman filter results are in Figure 6.6. Even though the lateral acceleration is missing, the sensor fusion data was able to approach the expected curve.
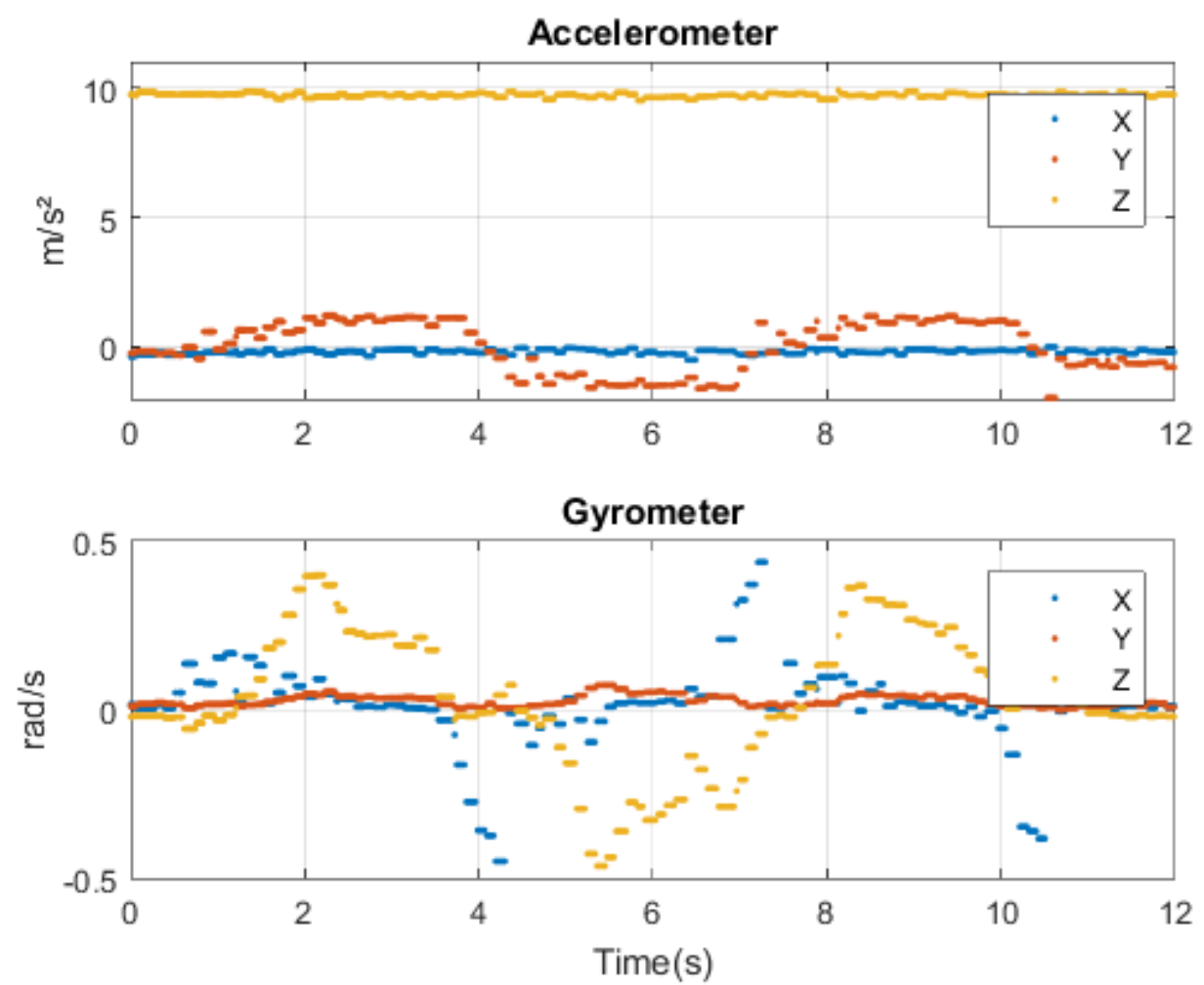

Figure 6.5 - Roll control measurements from IMU. 

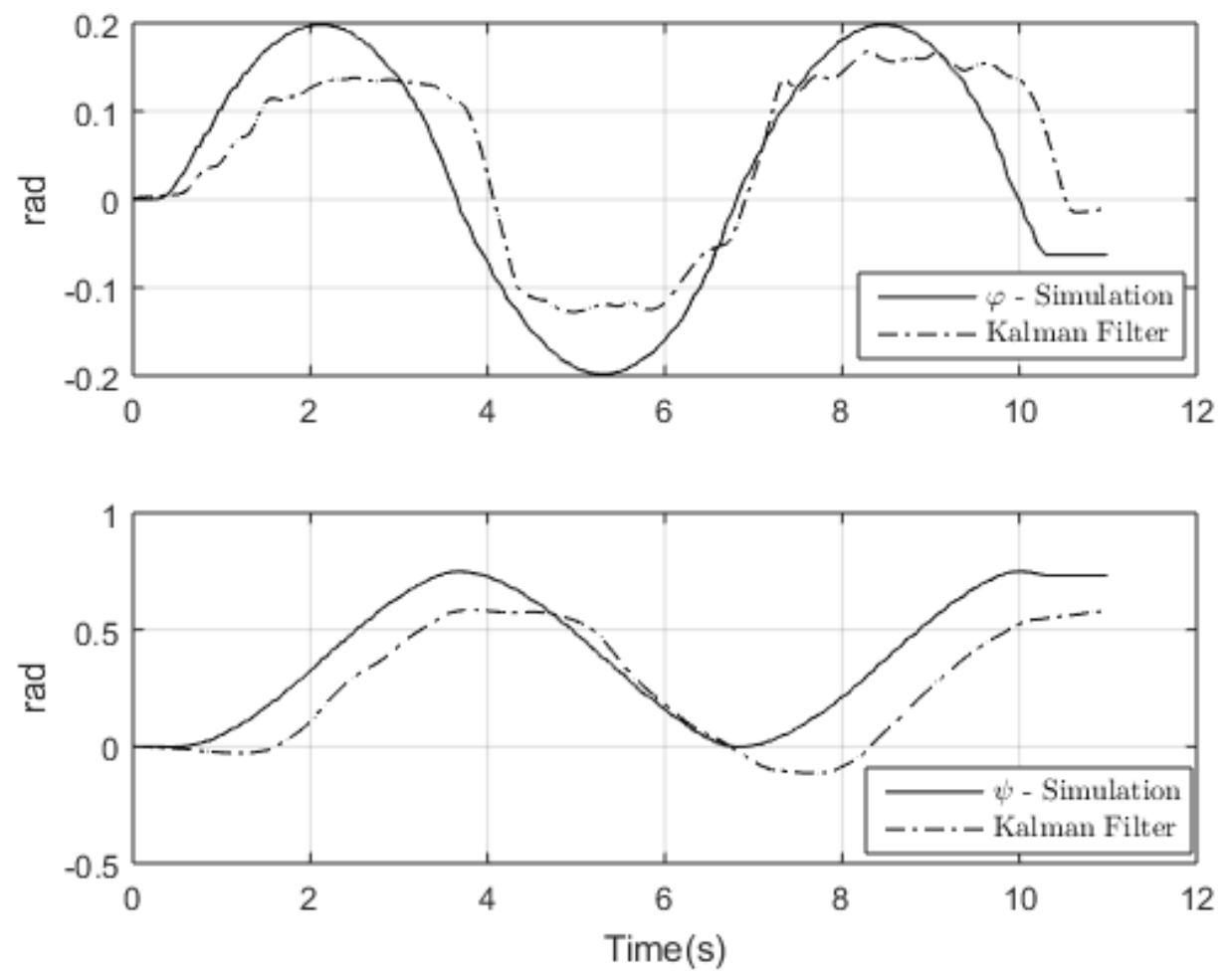

Figure 6.6 -Simulation versus measurements for roll control.

On the second test, a new target path was simulated, respecting the lateral speed and position boundaries. The simulated trajectory and respective angles performance for one of this tests are exhibited in Figure 6.7 and Figure 6.8, where the motorcycle follow through the complete track with maximum lateral speed, while at the highest longitudinal speed $(15 \mathrm{~m} / \mathrm{s})$. From the graphics, it is clear the roll and yaw angles will not be visually observable, since they are under one degree (0.018 rad).

The results measured from the motorcycle's embedded instrumentation are shown in Figure 6.9: as expected, the angular speeds are not measured by the sensors, since its small values are in the same magnitude of noises. The accelerometer, on the other hand, was not capable of measuring the lateral acceleration: analyzing the target path, the maximum lateral acceleration is $0.03 \mathrm{~m} / \mathrm{s}^{2}$, while the minimum detectable acceleration from the sensors is $0.6 \mathrm{~m} / \mathrm{s}^{2}$. Thus, on the present conditions, the maximum speed of the platform is not measured by the IMU. 

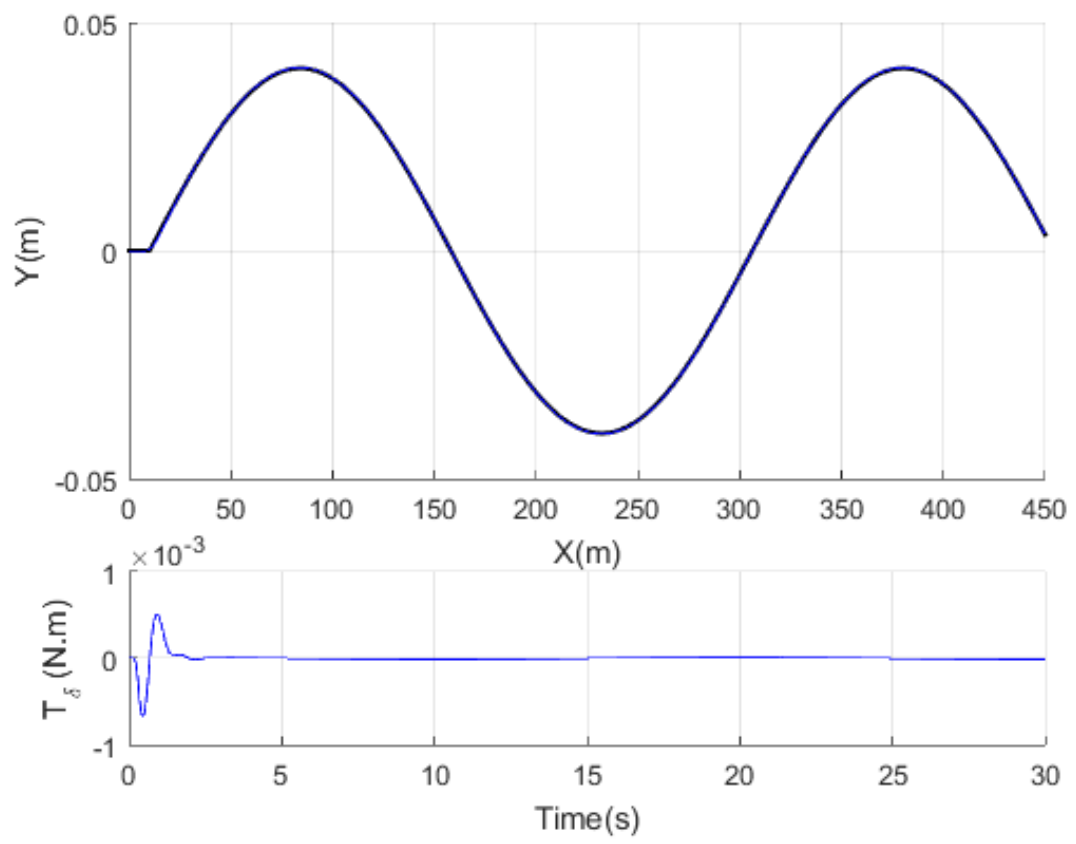

Figure 6.7 - Trajectory and torque on path tracking simulation for platform.
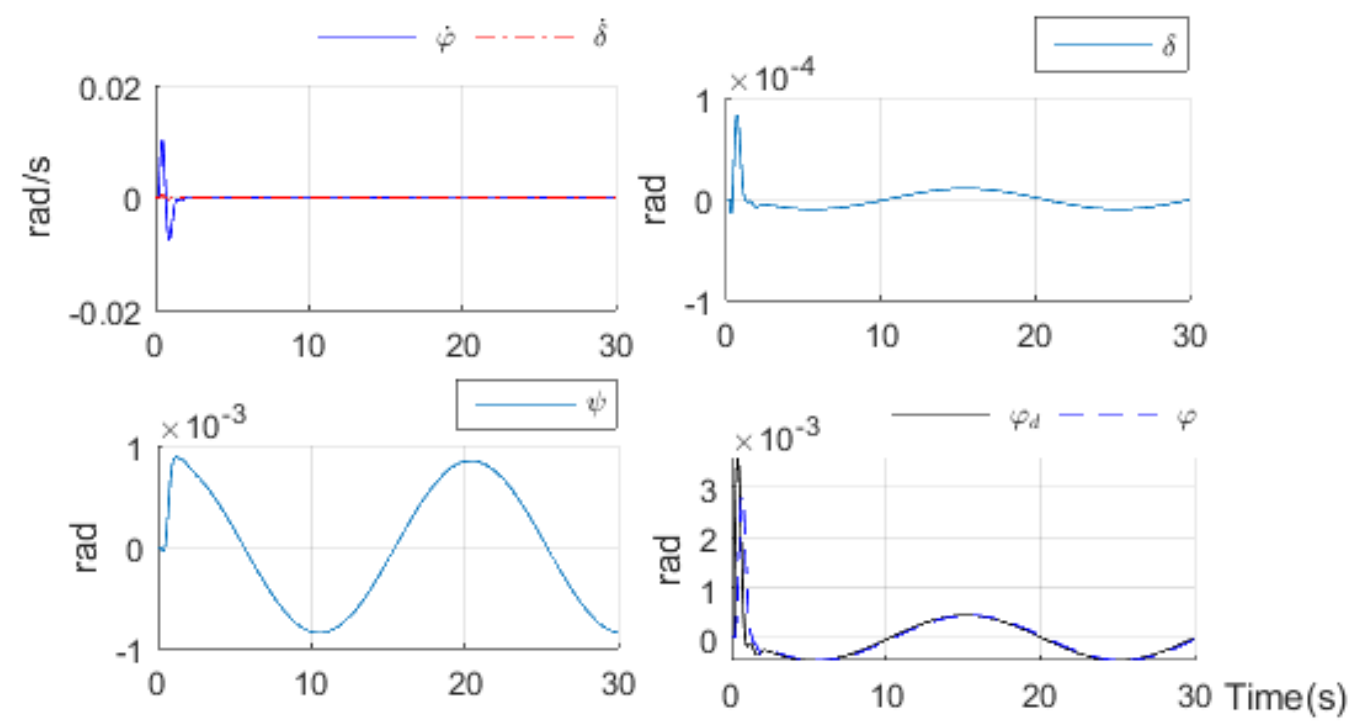

Figure 6.8 - Angular speeds and positions on path tracking simulation for platform. 

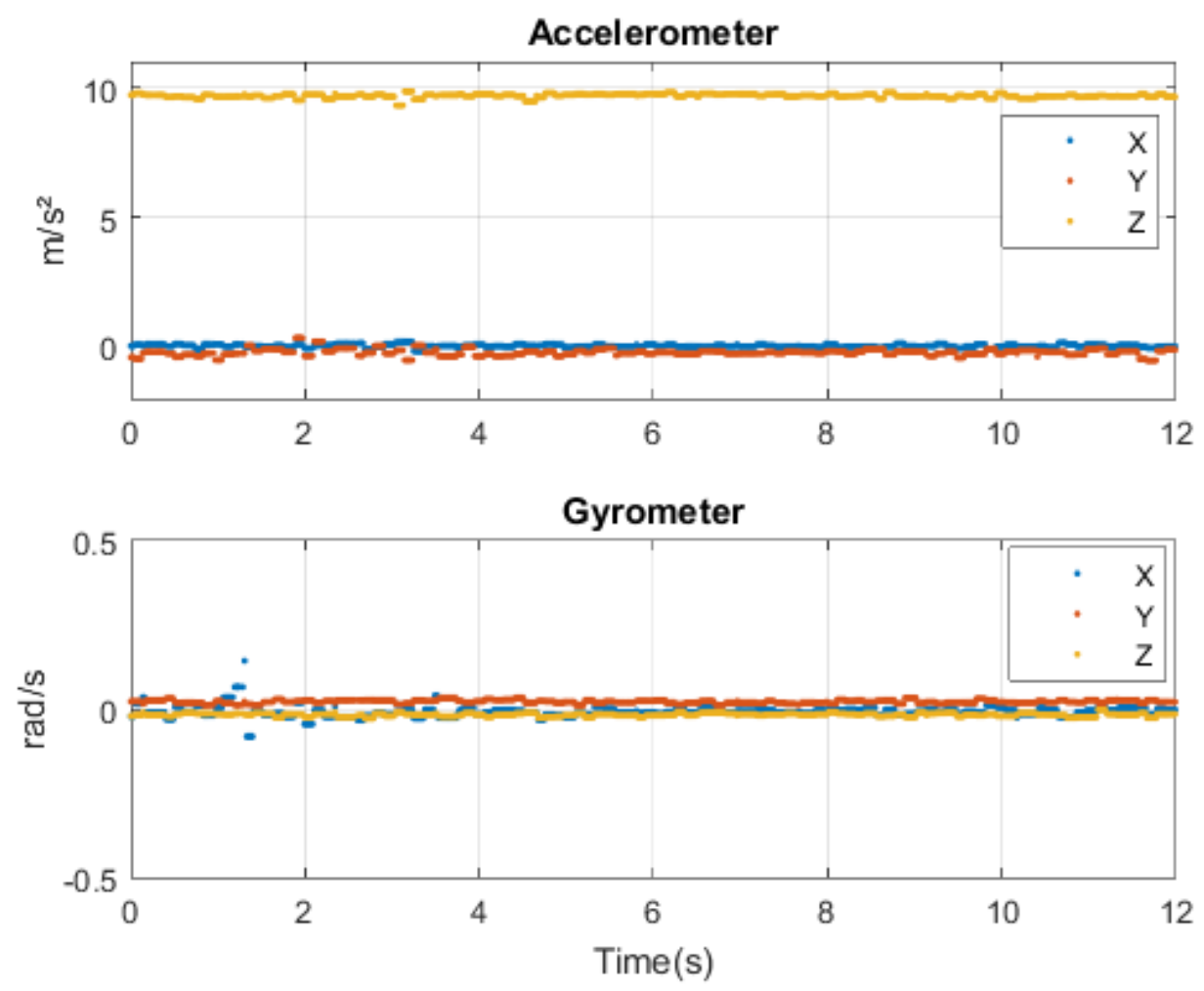

Figure 6.9 - Path tracking measurements from IMU.

This chapter presented an actuated platform, designed to test control algorithms for the autonomous motorcycle in a risk-free environment. The platform has three degrees of freedom, being able to reproduce roll and yaw movements, as well as lateral displacement. All movements are actuated by electric motors, with the angular motions being operated by servomotors while the lateral displacement is actuated by a DC motor.

This hardware-in-the-loop simulator integrates dynamic simulation (performed previously with Matlab) with real-time motion. A Labview program is responsible for integrating the simulation, sending the signals to the electric motors (via Arduino) and the data obtained by the instrumentation system, embedded on the motorcycle.

Experimental results with the roll and yaw movements prove the apparatus is capable of reproducing this dynamic behavior of two-wheeled vehicles. However, the lateral speed and displacement provided by the simulator was smaller than the expected by the dynamic model and could be improved in a next prototype, to reproduce more realistic movements. 


\section{$7 \quad$ Conclusions}

This work has presented a linear dynamic model of a two-wheeled vehicle. First, the geometric and inertial properties of a small scale electric motorcycle have been determined through a computational model and verified with an experiment; since the centers of mass position were equivalent, the numeric model was deemed representative of the studied object.

Then, the electric propulsion and steer systems were characterized: for the first motor, a relation between input signal and final rear wheel longitudinal speed was determined, based on the measurements of a tachometer connected to the motor's electric phases; the second system yielded a function between input signal and resulting steer angle, measured by a potentiometer embedded to the handlebar. Both relations are fundamental to the motorcycle dynamical model, playing a vital role to the vehicle stability and the control strategies.

Next, the unmanned single-track vehicle is analyzed, to verify the influence of design parameters and longitudinal speed to the vehicle selfstability. Unlike normal sized bicycles studied by Meijaard, the chosen electric motorcycle does not present a self-stable velocity range, which is attributed to its singular design, with a heavier rear wheel.

To solve the motorcycle stability problem and to guarantee a path trajectory, five control strategies were numerically analyzed. The first two focused exclusively on keeping the vehicle upright, without any considerations to the trajectory executed; one of the models simulated a more plausible environment by not considering all state variables directly measured by sensors. Both strategies were able to maintain the roll angle of the motorcycle under 15 degrees, respecting the small angle hypothesis of the considered linear model.

The remaining three control strategies focused on following a target path while also keeping the motorcycle stable. The best performance came from the preview path controller, in which the error of future positions is considered in the feedback loop. This strategy approaches a human behavior, unlike the other two algorithms, which passively responded to the difference between actual and desired positions. 
Once the control strategy to the autonomous motorcycle has been adjusted, it is necessary to implement it on the embedded instrumentation system. Since not all state variables are directly measured by sensors, and some sensors provide redundant data, it is necessary to apply a sensor fusion strategy. The chosen method was the classic Kalman filter approach, capable of attenuating noises and estimating parameters according to a given model. The numerical result was satisfactory, but with an expected drift due to the lack of position sensors; this estimate could be improved with dynamic equations for the lateral movement, in lieu of the kinematic relations used in this work.

At last, an actuated platform was built to test the control and sensor fusion strategies on a risk-free environment. Following the hardware in the loop simulation approach, the project is able to reproduce roll, yaw and small lateral displacements with the motorcycle. Though the prototype can benefit from upgrades, its performance was enough to visually demonstrate the most characteristic motions of single-track vehicles.

\subsection{Main contributions}

The dynamic modelling of two-wheeled vehicles has intrigued scientists since the nineteenth century, with the Whipple model, having gained greater repercussion since the seventies with the increase of ecological consciousness and the search for alternative transports. Thus, it was not the scope of this work to develop a new multibody model for motorcycles; the contribution of this thesis is to apply this widespread knowledge in a small scale system and verify its representativeness on this type of vehicle.

The use of model building technology is noteworthy, as previous works studied ridable bicycles or motorcycles; the small scale electric motorcycle analyzed in this work can be equated to conventional two-wheeled vehicles and the developed control strategies resemble the techniques applied to the former.

Additionally, the pieces of equipment developed to characterize the scale system - namely, the center of mass measurer, IMU measurer and dynamometer - also use model building technology and are interesting study objects for engineering teaching. Previous authors reinforced the importance of using familiar systems as study objects to engage students' curiosity; this work contributed with three scale low-cost equipment, which are easily manipulated by different levels students and could be reused in other projects. 
Still focusing on using this thesis as an educational object, the control strategies developed by this work present an increasing complexity, in order to engage students. Regarding the stability control, previous works have studied linear control approaches such as proportional-integration-derivative feedback, though not with the full state feedback, as developed by this thesis. This work also contributed with the use of a minimum-order observer to estimate the variables not directly measured by sensors.

As for the trajectory control strategies, this work contributed to the full state feedback control by aiming to guarantee the same dynamic behavior independently of the speed, with the same desired eigenvalues to the pole placement technique. Another addition to the literature is the linear quadratic regulator applied to the linear model using exclusively the steering torque input.

The sensor fusion strategy based on the use of low cost inertial measurement units brings economic advantages in the instrumentation costs and innovation. This work contributed to the study of the use of Kalman filters with an accelerometer and gyrometer to additionally obtain the motorcycle position in the plane, without the aid of supplementary devices such as GPS, computer vision or external locators.

Finally, this work developed a hardware-in-the-loop simulator; the scale actuated platform, besides aiding to visually verify the control and sensor fusion strategies implemented, may also serve as a teaching object to several undergraduate and graduate students, in the Mechanical and Control and Automation fields. The experimental bench is complex enough to allow three degrees of freedom motions, which can represent several vehicles, such as cars, bicycles or ships, while its graphic and real visualization simplifies to the students the dynamics happening in the background.

\subsection{Future works}

In order to enhance the linear model used in this work, it would be interesting to identify the behavior of the motorcycle tire, in order to add the drag motion between wheels in the predictions of the dynamic model. With this information, the model developed on Speranza Neto, M., Assad, M. M., Medeiros, V.S (2017) could be applied to the studied small scale motorcycle. This test would also require new experimental benches, such as a platform to identify the wheels characteristics, which can evolve to a test rig to analyze the gyroscopic effect on single-track vehicles. 
Similarity tests with real motorcycles or bicycles could also benefit the work, ensuring that the control strategies developed for the small scale system would also be successful in real equipment, if a known proportion between the two systems is observed. The opposite would also be true, in the sense that known parameters of real motorcycles could be applied on the small scale system without the need for further tests and experiments.

The control strategies analysis could be expanded to a nonlinear model, in order to account for more interesting phenomena, such as tire slippage or lateral dynamics. A feedback linearization technique could be used to transform the nonlinear system into an equivalent linear system and then utilize the proposed control strategies; otherwise, a gain scheduling approach could apply linear controllers to different operating points of the system.

The sensor fusion technique with inertial units to identify the plane position was not observed in other works in the area, which opens space for new analyzes. The system drift should be investigated and attenuated by other low cost solutions, keeping in mind the reduced dimensions of the system. A nonlinear approach to the problem could also be considered, such as an extended or unscented Kalman filter.

Lastly, the actuated platform could benefit from two main improvements: a larger lateral displacement course and a real time interaction between the simulation plant and the physical sensors. At the moment, the numerical simulation is done offline: Matlab calculates the expected motions by the motorcycle, which is then reproduced by the test rig, and checked with the instrumentation system. A larger track could allow more sudden and realistic movements.

\subsection{Thesis publications}

During the development of this work, four conference papers were published. With the financial support of Conselho Nacional de Desenvolvimento Científico e Tecnológico (CNPq), a paper was presented in the international conference Diname, held in São Sebastião, Brazil.

ASSAD, M. M.; SPERANZA NETO, M. Small Scale Motorcycle as Educational and Research Engineering Tool. In $11^{\text {th }}$ International Technology, Education and Development Conference, pp. 4656-4665, Valencia, Spain, 2017. 
ASSAD, M. M.; SPERANZA NETO, M.; MEGGIOLARO, M. A. Analysis of control strategies for autonomous motorcycles stabilization and trajectories tracking. In XVII International Symposium on Dynamic Problems of Mechanics, São Paulo, Brazil, 2017.

SPERANZA NETO, M.; ALBUQUERQUE, A. N.; ASSAD, M. M. Small Scale Mechatronics Devices as Educational and Research Engineering Tools. . In $11^{\text {th }}$ Symposium on Advances in Control Education, Volume 49, Number 6, pp. 248-255, Bratislava, Slovakia, 2016.

SPERANZA NETO, M.; ASSAD, M. M.; MEDEIROS, V.S. Bicycles and motorcycles as an educational object for modeling, analysis and simulation of stability, speed, suspensions, attitude and trajectory control. In $24^{\text {th }}$ International Congress of Mechanical Engineering, Curitiba, Brazil, 2017.

Another conference paper has been developed besides the work of the thesis, together with other two graduate students.

COSTA, M. S. M.; MEGGIOLARO, M. A.; SPERANZA NETO, M., Albuquerque, A. N.; ASSAD, M. M. Performance evaluation of a sensor fusion algorithm for attitude estimation using commercial IMU and a scale Stewart platform. In $23^{\text {rd }}$ International Congress of Mechanical Engineering, Rio de Janeiro, Brazil, 2015.

Lastly, the following journal paper has been accepted by the $A B C M$ Series.

ASSAD, M. M.; SPERANZA NETO, M.; MEGGIOLARO, M. A. Analysis of control strategies for autonomous motorcycles stabilization and trajectories tracking. In $\mathrm{ABCM}$ Series on Mechanical Sciences and Engineering - Proceedings of DINAME 2017, Springer, 2017. 


\section{Bibliography}

ABYARJOO, F.; BARRETO, A.; COFINO, J.; ORTEGA, F. R. Implementing a sensor fusion algorithm for 3D orientation detection with inertial/magnetic sensors. In Innovations and Advances in Computing, Informatics, Systems Sciences, Networking and Engineering, pp. 305-310, Springer, 2015.

ALATISE, M. B.; HANCKE, G. P. Pose estimation of a mobile robot based on fusion of IMU data and vision data using an extended Kalman filter. Sensors, Volume 17, Number 10, pp. 2164, 2017.

ARDEMA, M. D. Newton-Euler Dynamics. $1^{\text {st }}$ Edition, Springer Science \& Business Media, 2005.

ASSAD, M. M.; SPERANZA NETO, M. Small Scale Motorcycle as Educational and Research Engineering Tool. In $11^{\text {th }}$ International Technology, Education and Development Conference, pp. 4656-4665, Valencia, Spain, 2017.

ASSAD, M. M.; SPERANZA NETO, M.; MEGGIOLARO, M. A. Analysis of control strategies for autonomous motorcycles stabilization and trajectories tracking. In XVII International Symposium on Dynamic Problems of Mechanics, São Paulo, Brazil, 2017.

ASSAD, M. M.; SPERANZA NETO, M.; MEGGIOLARO, M. A. Analysis of control strategies for autonomous motorcycles stabilization and trajectories tracking. In $\mathrm{ABCM}$ Series on Mechanical Sciences and Engineering - Proceedings of DINAME 2017, Springer, 2017

ASTRÖM, K.J.; KLEIN, R.E.; LENNARTSSON, A. Bicycle dynamics and control, adapted bicycles for education and research. In IEEE Control Systems, Volume 25, Number 4, pp. 26-47, 2005.

ASTRÖM, K. J.; MURRAY, R. M. Feedback systems: an introduction for scientists and engineers. Princeton University Press, 2010. 
BARSHAN, B.; DURRANT-WHYTE, H. F. Inertial navigation systems for mobile robots. In IEEE Transactions on Robotics and Automation, Volume 11, Number 3, pp. 328-342, 1995.

BEZNOS, A. V., FORMAL'SKY, A. M.; GURFINKEL, E. V.; JICHAREV, D. N.; LENSKY, A. V.; SAVITSKY, K. V.; TCHESALIN, L. S. Control of autonomous motion of two-wheel bicycle with gyroscopic stabilization. In International Conference on Robotics and Automation, Volume 3, pp. 2670-2675, Leuven, Belgium, 1998.

BLOIS, P.F.C. Controle de atitude de um veículo robótico elétrico em fase balística (in Portuguese), PhD Thesis, Pontifical Catholic University, Rio de Janeiro, Brazil, 2013.

BONIOLO, I.; SAVARESI, S. M.; TANELLI, M. Roll angle estimation in twowheeled vehicles. In IET Control Theory \& Applications, Volume 3, Number 1, pp. 20-32, 2009.

BONIOLO, I.; CORBETTA, S.; SAVARESI, S. M. Attitude estimation of a motorcycle in a Kalman filtering framework. In International Federation of Automatic Control, Volume 43, Number 7, pp. 779-784, 2010.

BONIOLO, I.; SAVARESI, S. M.; TANELLI, M. Lean angle estimation in twowheeled vehicles with a reduced sensor configuration. In International Symposium on Circuits and Systems, pp. 2573-2576, Seoul, Korea, 2012.

CAICEDO, Y. E. C.; QUINTERO, C. H. L.; DE BRITTO VIDAL FILHO, W. A study of three control approaches for the cyclist robot problem. In $20^{\text {th }}$ International Congress of Mechanical Engineering, Rio Grande do Sul, Brazil, 2009.

CAIN, S. M.; PERKINS, N. C. Comparison of a bicycle steady-state turning model to experimental data. In Symposium on the Dynamics and Control of Single Track Vehicles, Delft, Netherlands, 2010.

CHATAIGNIER, P.S. Desenvolvimento de um sistema de obtenção de dados para calibração de centrais inerciais (in Portuguese). Undergraduate Research, Control and Automation Engineering, Pontifical Catholic University, Rio de Janeiro, Brazil, 2014. 
CHEN, C. K.; DAO, T. S. Fuzzy control for equilibrium and roll-angle tracking of an unmanned bicycle. In Multibody system dynamics, Volume 15, Number 4, pp. 325-350, 2006.

CHEN, C. K.; DAO, T. S. Genetic fuzzy control for path-tracking of an autonomous robotic bicycle. In Journal of system design and dynamics, Volume 1, Number 3, pp. 536-547, 2007.

CHEN, C. K.; DAO, T. K. A study of bicycle dynamics via system identification. In International Symposium on Computer, Communication Control and Automation, Volume 2, pp. 204-207, 2010.

CHENANE, C.; ICHALAL, D.; ARIOUI, H.; MAMMAR, S. Lateral dynamics reconstruction for sharp'71 motorcycle model with p2i observer. In $2^{\text {nd }}$ International Conference on Systems and Control, pp. 1-6, Marrakech, Morocco, 2012.

CHENANE, C.; ICHALAL, D.; ARIOUI, H.; MAMMAR, S. Proportional two integral (P2I) observer synthesis for single track vehicle. In $20^{\text {th }}$ Mediterranean Conference on Control \& Automation, pp. 1530-1535, Barcelona, Spain, 2012.

CORBETTA, S.; BONIOLO, I.; SAVARESI, S. M. Attitude estimation of a motorcycle via unscented Kalman filter. In $5^{\text {th }}$ IFAC Symposium on Mechatronic Systems, Volume 43, Number 18, pp. 511-516, Massachusetts, USA, 2010.

COSSALTER, V., LOT, R. <www.dynamotion.it/eng/dinamoto/8_online_papers/wobble-weave/wobble-weave_eng.html> Accessed in October, 2014.

COSSALTER, V. Motorcycle Dynamics. 2nd Edition, Lulu.com, 2006

COSTA, M.S.M. Controle de veículos aéreos quadrirotores: uso de filtros de Kalman para minimização de erros na unidade de medida inercial. (In Portuguese), Masters Dissertation, Pontifical Catholic University, Rio de Janeiro, Brazil, 2014. 
COSTA, M. S. M.; MEgGIOLARO, M. A.; SPERANZA NETO, M.; ALBUQUERQUE, A. N.; ASSAD, M. M. Performance evaluation of a sensor fusion algorithm for attitude estimation using commercial IMU and a scale Stewart platform. In 23nd International Congress of Mechanical Engineering, Rio de Janeiro, Brazil, 2015.

DAO, T. K.; CHEN, C. K. Path tracking control of a motorcycle based on system identification. In IEEE Transactions on Vehicular Technology, Volume 60, Number 7, pp. 2927-2935, 2011.

DAO, T. K.; CHEN, C. K. Path-tracking control of a riderless bicycle via road preview and speed adaptation. In Asian Journal of Control, Volume 15, Number 4, pp. 1036-1050, 2013.

ESCALONA, J. L.; RECUERO, A. M. A bicycle model for education in machine dynamics and real-time interactive simulation. In Symposium on the Dynamics and Control of Single Track Vehicles, pp. 20-32, Delft, Netherlands, 2010.

FAJANS, J. Steering in bicycles and motorcycles. In American Journal of Physics, Volume 68, Number 7, pp. 654-659, 2000.

FREZZA, R.; BEGHI, A.; SACCON, A. Model predictive for path following with motorcycles: application to the development of the pilot model for virtual prototyping. In 43rd IEEE Conference on Decision and Control, pp. 767-772, Paradise Island, Bahamas, 2004.

GALLASPY, J. M.; HUNG, J. Y. Gyroscopic stabilization of a stationary unmanned bicycle. Technical Report, Auburn University, Alabama, USA, 1999.

GASBARRO, L.; BEGHI, A.; FREZZA, R.; Nori, F.; SPAGNOL, C. Motorcycle trajectory reconstruction by integration of vision and MEMS accelerometers. In 43rd IEEE Conference on Decision and Control, pp. 779783, Paradise Island, Bahamas, 2004.

GETZ, N. H.; MARSDEN, J. E. Control for an autonomous bicycle. In IEEE International Conference on Robotics and Automation, Volume 2, pp. 13971402, Nagoya, Japan, 1995. 
GETZ, N. H. Internal equilibrium control of a bicycle. In 34th IEEE Conference on Decision and Control, Volume 4, pp. 4285-4287, Louisiana, USA, 1995.

GOEL, P.; ROUMELIOTIS, S. I.; SUKHATME, G. S. Robust localization using relative and absolute position estimates. In International Conference on Intelligent Robots and Systems, pp. 1134-1140, Kyongju, South Korea, 1999.

GREWAL, M. S.; HENDERSON, V. D.; MIYASAKO, R. S. Application of Kalman filtering to the calibration and alignment of inertial navigation systems. In IEEE Transactions on Automatic Control, Volume 36, Number 1, pp. 3-13, 1991.

GREWAL, M. S.; ANDREWS, A. P. Kalman filtering: theory and practice using MATLAB, $2^{\text {nd }}$ edition, John Wiley \& Sons, 2001.

GUARNIERI, A.; PIROTTI, F.; VETTORE, A. Low-cost MEMS sensors and vision system for motion and position estimation of a scooter. In Sensors, Volume 13, Number 2, pp. 1510-1522, 2013.

GUNEY, S.; ATASOY, A. An approach to pole placement method with output feedback. Technical Report, Karadeniz Technical University, Trabzon, Turkey, 2011.

GUPTA, V.; BRENNAN, S. Terrain-based vehicle orientation estimation combining vision and inertial measurements. In Journal of Field Robotics, Volume 25, Number 3, pp. 181-202, 2008.

HELLMERS, H.; NORRDINE, A.; BLANKENBACH, J.; EICHHORN, A. An IMU/magnetometer-based indoor positioning system using Kalman filtering. In $4^{\text {th }}$ International Conference on Indoor Positioning and Indoor Navigation, pp. 1-9, Montbéliard, France, 2013.

HESS, R.; MOORE, J. K.; HUBBARD, M. Modeling the manually controlled bicycle. In IEEE Transactions on Systems, Man, and Cybernetics - Part A: Systems and Humans, Volume 42, Number 3, pp. 545-557, 2012.

HIGGINS, W. T. A comparison of complementary and Kalman filtering. In IEEE Transactions on Aerospace and Electronic Systems, Number 3, pp. 321325, 1975. 
HUBBARD, M.; HESS, R.; MOORE, J. K.; PETERSON, D. L. Human control of bicycle dynamics with experimental validation and implications for bike handling and design. In 2011 NSF Engineering Research and Innovation Conference, Georgia, USA, 2011.

HUYGE, K.; AMBRÓSIO, J.; PEREIRA, M. A control strategy for the dynamics of a motorcycle, including rider. In $5^{\text {th }}$ EUROMECH Nonlinear Oscillations Conference, Eindhoven, Netherlands, 2005.

HUYGHE, B.; DOUTRELOIGNE, J.; VANFLETEREN, J. 3D orientation tracking based on unscented Kalman filtering of accelerometer and magnetometer data. In Sensors Applications Symposium, pp. 148-152, Louisiana, USA, 2009.

KAPANIA, N. R.; GERDES, J. C. Design of a feedback-feedforward steering controller for accurate path tracking and stability at the limits of handling. In Vehicle System Dynamics, Volume 53, Number 12, pp. 1687-1704, 2015.

KELLY, A. A 3D state space formulation of a navigation Kalman filter for autonomous vehicles, Masters Dissertation, Carnegie-Mellon University, Pittsburgh, USA, 1994.

KEO, L.; MASAKI, Y. Trajectory control for an autonomous bicycle with balancer. In International Conference on Advanced Intelligent Mechatronics, pp. 676-681, Xi'an, China, 2008.

KEO, L.; YAMAKITA, M. Controller design of an autonomous bicycle with both steering and balancer controls. In $18^{\text {th }}$ IEEE International Conference on Control Applications, pp. 1294-1299, Saint Petersburg, Russia, 2009.

KEO, L.; YOSHINO, K.; KAWAGUCHI, M.; YAMAKITA, M. Experimental results for stabilizing of a bicycle with a flywheel balancer. In International Conference on Robotics and Automation, pp. 6150-6155, Shanghai, China, 2011.

KIM, J.; LEE, S. A vehicular positioning with GPS/IMU using adaptive control of filter noise covariance. In ICT Express, Volume 2, Number 1, pp. 41-46, 2016. 
KIM, P. Kalman filter for beginners: with MATLAB examples. $1^{\text {st }}$ Edition, CreateSpace, 2011.

KING, P. H. A low cost localization solution using a Kalman filter for data fusion. Masters Dissertation, Virginia Polytechnic Institute, Virginia, USA, 2008.

KLEIN, R. E. Using bicycles to teach system dynamics. In IEEE Control Systems Magazine, Volume 9, Number 3, pp. 4-9, 1989.

KOOIJMAN, J. D. G.; SCHWAB, A. L.; MEIJAARD, J. P. Experimental validation of a model of an uncontrolled bicycle. In Multibody System Dynamics, Volume 19, pp. 115-132, 2008.

KOOIJMAN, J. D. G.; SCHWAB, A. L. A review on handling aspects in bicycle and motorcycle control. In International Design Engineering Technical Conference \& Computers and Information in Engineering, pp. 597607, Washington D.C., USA, 2011

KOOIJMAN, J. D. G.; MEIJAARD, J. P.; PAPADOPOULOS, J. M.; RUINA, A.; SCHWAB, A. L. A bicycle can be self-stable without gyroscopic or caster effects. In Science, Volume 332, pp. 339-342, 2011.

KOOIJMAN, J. D. G. Bicycle rider control: observations, modeling \& experiments. PhD Thesis, Delft University of Technology, Delft, Netherlands, 2012.

LANGE, P.D.L. Rider control identification in bicycling. Masters Dissertation, Delft University of Technology, Delft, Netherlands, 2011.

LECCADITO, M. A Kalman Filter Based Attitude Heading Reference System Using a Low Cost Inertial Measurement Unit. Masters Dissertation, Virginia Commonwealth University, Virginia, USA, 2013

LEE, T.; SHIN, J.; CHO, D. Position Estimation for Mobile Robot Using Inplane 3-Axis IMU and Active Beacon. In IEEE International Symposium on Industrial Electronics, pp. 1956-1961, Seoul, South Korea, 2009.

LEUNG, K. T.; WHIDBORNE, J. F.; PURDY, D.; BARBER, P. Road vehicle state estimation using low-cost GPS/INS. In Mechanical Systems and Signal Processing, Volume 25, Number 6, pp. 1988-2004, 2011. 
LI, W.; QUIROGA Garcia, P. E. On Indoor Positioning for Mobile Devices. Masters Dissertation, Chalmers University of Technology, Göteborg, Sweden, 2011.

LIMEBEER, D. J.; SHARP, R. S. Bicycles, Motorcycles, and Models. In IEEE Control Systems Magazine, Volume 26, Number 5, pp. 34-61, 2006.

LOWELL, J.; MCKELL, H. D. The stability of bicycles. In American Journal of Physics, Volume 50, Number 12, pp. 1106-1112, 1982.

MADGWICK, S. O.; HARRISON, A. J.; VAIDYANATHAN, R. Estimation of IMU and MARG orientation using a gradient descent algorithm. In IEEE International Conference on Rehabilitation Robotics, pp. 1-7, Zurich, Switzerland, 2011.

MAKLOUF, O.; GHILA, A.; ABDULLA, A. Cascade Kalman Filter Configuration for Low Cost IMU/GPS Integration in Car Navigation Like Robots. In International Journal of Electrical, Computer, Energetic, Electronic and Communication Engineering, Volume 6, Number 6, pp. 571-578, 2012.

MALVEZZI, M., VETTORI, G., ALlOTTA, B., PUGI, L., RIDOLFI, A.; RINDI, A. A localization algorithm for railway vehicles based on sensor fusion between tachometers and inertial measurement units. In Proceedings of the Institution of Mechanical Engineers, Part F: Journal of Rail and Rapid Transit, Volume 228, Number 4, pp. 431-448, 2014.

MARLIN, T. E. Process Control: designing processes and control systems for dynamic performance. $2^{\text {nd }}$ Edition, New York: McGraw-Hill, 1995.

MARTINS, G. N. Concepção e Modelagem de Objetos Educacionais para Compreensão da Dinâmica de Motocicletas/Bicicletas em Projetos de Engenharia (in Portuguese). PhD Thesis, Pontifical Catholic University, Rio de Janeiro, Brazil, 2016.

MEIJAARD, J. P.; PAPADOPOULOS, J. M.; RUINA, A.; SCHWAB, A. L. Linearized dynamics equations for the balance and steer of a bicycle: a benchmark and review. In Proceedings of the Royal Society of London A: Mathematical, Physical and Engineering Sciences, Volume 463, Number 2084, pp. 1955-1982, 2007. 
MOORE, J. K.; HUBBARD, M.; SCHWAB, A. L.; KOOIJMAN, J. D. Accurate Measurement of Bicycle Parameters. In Symposium on the Dynamics and Control of Single Track Vehicles, Delft, Netherlands, 2010.

OGATA, K., YANG, Y. Modern Control Engineering, $5^{\text {th }}$ Edition. Prentice Hall, 2010.

OTT, C., ANGROSCH, B., EDELMANN, J.; PLÖCHL, M. Some Investigations on the Wobble Mode of a Bicycle. In Symposium on the Dynamics and Control of Single Track Vehicles, Delft, Netherlands, 2010.

PAPADOPOULOS, J. M. Bicycle steering dynamics and self-stability: a summary report on work in progress. Cornell Bicycle Research Project, Cornell University, pp. 1-23, 1987.

PLÖCHL, M.; EDELMANN, J.; ANGROSCH, B.; OTT, C. On the wobble mode of a bicycle. In Vehicle System Dynamics, Volume 50, Number 3, pp. 415-429, 2012.

QUANSER. Quanser Innovate Educate: user case studies. <http://www.quanser.com/case_studies>. Ontario, Canada. Accessed in June, 2017.

RAJAMANI, R. Vehicle Dynamics and Control. $2^{\text {nd }}$ Edition, Mechanical Engineering Series, Springer, 2012.

RANDLOV, J.; ALSTROM, P. Learning to Drive a Bicycle using Reinforcement Learning and Shaping. In International Conference on Machine Learning, Volume 98, pp. 463-471, Wisconsin, USA, 1998.

REHBINDER, H.; HU, X. Drift-free attitude estimation for accelerated rigid bodies. In Automatica, Volume 40, Number 4, pp. 653-659, 2004.

REHM, A. Estimation of Vehicle Roll Angle. In $4^{\text {th }}$ IEEE International Symposium on Communications, Control and Signal Processing, pp. 1-4, Limassol, Cyprus, 2010. 
RENAUDIN, V.; COMBETTES, C. Magnetic, Acceleration Fields and Gyroscope Quaternion (MAGYQ) - Based Attitude Estimation with Smartphone Sensors for Indoor Pedestrian Navigation. In Sensors, Volume 14, Number 12, pp. 22864-22890, 2014.

RIBEIRO, M. I. Kalman and Extended Kalman Filters: Concept, Derivation and Properties. In Institute for Systems and Robotics, Volume 43, 2004.

RINGWOOD, J.; FENG, R. Bicycle Wheel Wobble: A Case Study in Dynamics. In International Conference on Informatics in Control, Automation and Robotics, pp. 238-243, Angers, France, 2007.

ROMANIUK, S.; GOSIEWSKI, Z. Kalman filter realization for orientation and position estimation on dedicated processor. In Acta Mechanica et Automatica, Volume 8, Number 2, pp. 88-94, 2014.

RYU, J. State and parameter estimation for vehicle dynamics control using GPS. PhD Thesis, Stanford University, California, USA, 2005.

SANTOS, I. F. Dinâmica de sistemas mecânicos (in Portuguese). $2^{\text {nd }}$ Edition, Ed. Makron Books, Sao Paulo, Brazil, 2001.

SCHIEHLEN, W. Dynamical analysis of vehicle systems: theoretical foundations and advanced applications. $1^{\text {st }}$ Edition. Ed. SpringerWienNewYork, 2009.

SCHLIPSING, M.; SALMEN, J.; LATTKE, B.; SCHRÖTER, K. G.; WINNER, H. Roll Angle Estimation for Motorcycles: Comparing Video and Inertial Sensor Approaches. In IEEE Intelligent Vehicles Symposium, Volume 4, pp. 500-505, Alcalá de Henares, Spain, 2012.

SCHMITT, T. Modeling of a Motorcycle in Dymola/Modelica. Masters Dissertation, Vorarlberg University of Applied Sciences, Dornbirn, Austria, 2009.

SCHMITT, T.; ZIMMER, D.; CELLIER, F. E. A Virtual Motorcycle Rider Based on Automatic Controller Design. In 7th International Modelica Conference, pp. 19-28, Como, Italy, 2009. 
SCHWAB, A. L.; MEIJAARD, J. P.; PAPADOPOULOS, J. M. Benchmark Results on the Linearized Equations of Motion of an Uncontrolled Bicycle. In Journal of Mechanical Science and Technology, Volume 19, pp. 292-304, 2005.

SCHWAB, A. L.; MEIJAARD, J. P. A review on bicycle dynamics and rider control. In Vehicle System Dynamics, Volume 51, Number 7, pp. 1059-1090, 2013.

SCHWAB, A. L.; DE LANGE, P. D. L.; HAPPEE, R.; MOORE, J. K. Rider control identification in bicycling using lateral force perturbation tests. In Institution of Mechanical Engineers, Part K: Journal of Multi-body Dynamics, Volume 227, Number 4, pp. 390-406, 2013.

SCHWAB, A. L.; RECUERO, A. M. Design and experimental validation of a haptic steering interface for the control input of a bicycle simulator. In ECCOMAS Multibody Dynamics, pp. 103-110, Zagreb, Croatia, 2013.

SHARP, R. S. The stability and control of motorcycles. In Journal of mechanical engineering science, Volume 13, Number 5, pp. 316-329, 1971.

SHARP, R. S. The lateral dynamics of motorcycles and bicycles. In Vehicle System Dynamics, Volume 14, pp. 265-283, 1985.

SHARP, R. S., and VALTETSIOTIS, V. Optimal Preview Car Steering Control. In Vehicle System Dynamics, Volume 35, pp. 101-117, 2001.

SHARP, R. S. Driver steering control and a new perspective on car handling qualities. In Proceedings of the Institution of Mechanical Engineers, Part C: Journal of Mechanical Engineering Science, Volume 219, Number 10, pp. 1041-1051, 2005.

SHARP, R. S. Motorcycle steering control by road preview. In Journal of Dynamic Systems, Measurement, and Control, Volume 129, pp. 373-381, 2007.

SILVA, M.R., RAFIKOVA, E. Android Based Sensor Fusion and Control Navigation Strategy for a Wheeled Mobile Robot. In XVII International Symposium on Dynamic Problems of Mechanics, São Paulo, Brazil, 2017. 
SIMON, D. Kalman Filtering. In Embedded Systems Programming, Volume 14, Number 6, pp. 72-79, 2001.

SIQUEIRA, R.G. Localização inercial de um veículo quatro rodas com esterçamento utilizando fusão sensorial (In Portuguese), Masters Dissertation, Instituto Militar de Engenharia, Rio de Janeiro, Brazil, 2012.

SPERANZA NETO, M., ALBUQUERQUE, A. N.; ASSAD, M. M. Small Scale Mechatronics Devices as Educational and Research Engineering Tools. In $11^{\text {th }}$ Symposium on Advances in Control Education, Volume 49, Number 6, pp. 248-255, Bratislava, Slovakia, 2016.

SPERANZA NETO, M., ASSAD, M. M., MEDEIROS, V.S. Bicycles and motorcycles as an educational object for modeling, analysis and simulation of stability, speed, suspensions, attitude and trajectory control. In $24^{\text {th }}$ International Congress of Mechanical Engineering, Curitiba, Brazil, 2017.

SURYANARAYANAN, S.; TOMIZUKA, M.; WEAVER, M. System dynamics and control of bicycles at high speeds. In American Control Conference, Volume 2, pp. 845-850, Alaska, USA, 2002.

TAK, T. O.; WON, J. S.; BAEK, G. Y. Design Sensitivity Analysis of Bicycle Stability and Experimental Validation. In Symposium on the Dynamics and Control of Single Track Vehicles, pp. 20-32, Delft, Netherlands, 2010.

TANAKA, Y.; MURAKAMI, T. Self Sustaining Bicycle Robot with steering controller. In $8^{\text {th }}$ IEEE International Workshop on Advanced Motion Control, pp. 193-197, Kawasaki, Japan, 2004.

TANAKA, Y.; MURAKAMI, T. A Study on Straight-Line Tracking and Posture Control in Electric Bicycle. In IEEE Transactions on Industrial Electronics, Volume 56, Number 1, pp. 159-168, 2009.

TANELLI, M.; CORNO, M.; SAVERESI, S. Modelling, simulation and control of two-wheeled vehicles. Automotive Series, $1^{\text {st }}$ Edition, John Wiley \& Sons, 2014.

TEERHUIS, A. P.; JANSEN, S. T. H. Motorcycle State Estimation for Lateral Dynamics. In Symposium on the Dynamics and Control of Single Track Vehicles, Delft, Netherlands, 2010. 
TRAWNY, N.; ROUMELIOTIS, S. I. Indirect Kalman Filter for 3D Attitude Estimation. Technical Report, Rev. 57, Number 2005-002, University of Minnesota, Minnesota, USA, 2005.

VAGANAY, J.; ALDON, M.J.; FOURNIER A. Mobile Robot Attitude Estimation by Fusion of Inertial Data. In IEEE International Conference on Robotics and Automation, Volume 1, pp. 277-282, Georgia, USA, 1993.

VALENTI, R. G.; DRYANOVSKI, I.; XIAO, J. Keeping a Good Attitude: A Quaternion-Based Orientation Filter for IMUs and MARGs. In Sensors, Volume 15, Number 8, pp. 19302-19330, 2015.

WIT, J. S. Vector pursuit path tracking for autonomous ground vehicles. PhD Thesis, University of Florida, Florida, USA, 2000.

WOODMAN, O. J. An introduction to inertial navigation. Technical Report, Number 696, University of Cambridge, Cambridge, United Kingdom, 2007.

YAMAKITA, M.; UTANO, A.; SEKIGUCHI, K. Experimental Study of Automatic Control of Bicycle with Balancer. In International Conference on Intelligent Robots and Systems, pp. 5606-5611, Beijing, China, 2006.

YI, J.; SONG, D.; LEVANDOWSKI, A.; JAYASURIYA, S. Trajectory Tracking and Balance Stabilization Control of Autonomous Motorcycles. In International Conference on Robotics and Automation, pp. 2583-2589, Florida, USA, 2006.

YI, J.; ZHANG, Y.; SONG, D. Autonomous Motorcycles for Agile Maneuvers, Part I: Dynamic Modeling. In $48^{\text {th }}$ IEEE Conference on Decision and Control, pp. 4613-4618, Shanghai, China, 2009.

YI, J.; ZHANG, Y.; SONG, D. Autonomous Motorcycles for Agile Maneuvers, Part II: Control Systems Design. In $48^{\text {th }}$ IEEE Conference on Decision and Control, pp. 4619-4624, Shanghai, China, 2009.

ZHANG, P.; GU, J.; MILIOS, E. E.; HUYNH, P. Navigation with IMU/GPS/Digital Compass with Unscented Kalman filter. In International Conference on Mechatronics \& Automation, pp. 1497-1502, Niagara Falls, Canada, 2005. 
ZHANG, Y.; YI, J. Dynamic Modeling and Balance Control of Human/Bicycle Systems. In International Conference on Advanced Intelligent Mechatronics, pp. 1385-1390, Montréal, Canada, 2010.

ZHANG, Y.; LI, J.; YI, J.; SONG, D. Balance Control and Analysis of Stationary Riderless Motorcycles. In International Conference on Robotics and Automation, pp. 3018-3023, Shanghai, China, 2011.

ZHANG, Y.; CHEN, K.; YI, J. Rider Trunk and Bicycle Pose Estimation With Fusion of Force/Inertial Sensors. In IEEE Transactions on Biomedical Engineering, Volume 60, Number 9, pp. 2541-2551, 2013.

ZHANG, Y. Modeling and control of single-track vehicles: A humanmachine-environment interactions perspective. PhD Thesis, Rutgers University, New Jersey, USA, 2014.

ZHAO, H.; WANG, Z. Motion Measurement using Inertial Sensors, Ultrasonic Sensors, and Magnetometers with Extended Kalman Filter for Data Fusion. In Sensors, Volume 12, Number 5, pp. 943-953, 2012.

ZHU, R.; SUN, D.; ZHOU, Z.; WANG, D. A linear fusion algorithm for attitude determination using low cost MEMS-based sensors. In Measurement, Volume 40, Number 3, pp. 322-328, 2007. 


\section{Appendix A. Coefficients of the linearized two- wheeled model equations}

The multibody model (Meijaard, 2007) is initially designed by obtaining the front and rear frames center of mass and inertias in respect to the chosen origin - the rear wheel and floor point contact, represented by $O$ in Figure 3.1. Considering a particle system, $P_{i}, i=1, \ldots, n$, each with $m_{i}$ mass and located with coordinates $r_{i}$, the global center of mass is defined as Eq. (A.1).

$R=\frac{1}{\sum_{i=1}^{n} m_{i}} \sum_{i=1}^{n} m_{i} r_{i}$

Thus, the front frame center of mass is a combination of the front wheel and steering system's centers of mass and the rear frame combines the main body and rear wheel. Eq. (A.2) to (A.4) bring, respectively, the front frame (subscript $f f$ ), rear frame (subscript $r f$ ) and motorcycle (subscript $t$ ) resulting mass and center of mass position on axis $x$ and $z$.

$$
\begin{array}{lcc}
m_{f f}=m_{s}+m_{f w} & x_{f f}=\frac{m_{s} x_{s}+w m_{f w}}{m_{f f}} & z_{f f}=\frac{m_{s} z_{s}-m_{f w} r_{f w}}{m_{f f}} \\
m_{r f}=m_{r w}+m_{m} & x_{r f}=\frac{m_{m} x_{m}}{m_{r f}} & z_{r f}=\frac{m_{m} z_{m}-m_{r w} r_{r w}}{m_{r f}} \\
m_{t}=m_{r f}+m_{f f} & x_{t}=\frac{m_{r f} x_{r f}+m_{f f} x_{f f}}{m_{t}} & z_{t}=\frac{m_{r f} z_{r f}+m_{f f} z_{f f}}{m_{t}}
\end{array}
$$

The moments and products of inertia of a multibody system can be redefined at the same reference point by the parallel axis theorem. Being $I_{i j}$ the inertial tensor calculated in the center of mass of a body with mass $M$, a new inertia tensor $J_{i j}$, displaced from its origin by a vector $R$, is calculated in Eq. (A.5). 


$$
\begin{array}{ll}
J_{i j}=I_{i j}+M\left(R^{2} \beta_{i j}-R_{i} R_{j}\right) & \\
R=R_{1} \hat{x}+R_{2} \hat{y}+R_{3} \hat{z} & \beta_{i j}= \begin{cases}1 & i=j \\
0 & i \neq j\end{cases}
\end{array}
$$

The moments of inertia and inertial products of the rear and front frames should be calculated in respect to their respective centers of mass - $\left(x_{r f}, z_{r f}\right)$ and $\left(x_{f f}, z_{f f}\right)$. Thus, it is necessary to move the inertia tensors of the four bodies to those two reference points, as explicit by the previous equation. Eq. (A.6) to (A.8) bring the results to the front frame in respect to the $x$ (subscript $x x$ ), $z$ (subscript $z z$ ) and combined axis (subscript $x z$ ), while Eq. (A.9) to (A.11), to the rear frame.

$$
\begin{aligned}
J_{f f_{x x}} & =J_{s_{x x}}+m_{s}\left(z_{s}-z_{f f}\right)^{2}+J_{f w_{x x}}+m_{f w}\left(-r_{f w}-z_{f f}\right)^{2} \\
& =J_{s_{x x}}+m_{s}\left(z_{s}-z_{f f}\right)^{2}+J_{f w_{x x}}+m_{f w}\left(r_{f w}+z_{f f}\right)^{2} \\
J_{f f_{z z}} & =J_{s_{z z}}+m_{s}\left(x_{s}-x_{f f}\right)^{2}+J_{f w_{z z}}+m_{f w}\left(w-x_{f f}\right)^{2} \\
J_{f f_{x z}} & =J_{s_{x z}}-m_{s}\left(x_{s}-x_{f f}\right)\left(z_{s}-z_{f f}\right)-m_{f w}\left(w-x_{f f}\right)\left(-r_{f w}-z_{f f}\right) \\
& =J_{s_{x z}}-m_{s}\left(x_{s}-x_{f f}\right)\left(z_{s}-z_{f f}\right)+m_{f w}\left(w-x_{f f}\right)\left(r_{f w}+z_{f f}\right) \\
J_{r f_{x x}} & =J_{m_{x x}}+m_{m}\left(z_{m}-z_{r f}\right)^{2}+J_{r w_{x x}}+m_{r w}\left(-r_{r w}-z_{r f}\right)^{2} \\
& =J_{m_{x x}}+m_{m}\left(z_{m}-z_{r f}\right)^{2}+J_{r w_{x x}}+m_{r w}\left(r_{r w}+z_{r f}\right)^{2} \\
J_{r f_{z z}} & =J_{m z z}+m_{m}\left(x_{m}-x_{r f}\right)^{2}+J_{r w_{z z}}+m_{r w}\left(0-x_{r f}\right)^{2} \\
& =J_{m z z}+m_{m}\left(x_{m}-x_{r f}\right)^{2}+J_{r w_{z z}}+m_{r w} x_{r f}^{2} \\
J_{r f_{x z}} & =J_{m_{x z}}-m_{m}\left(x_{m}-x_{r f}\right)\left(z_{m}-z_{r f}\right)-m_{r w}\left(0-x_{r f}\right)\left(-r_{r w}-z_{r f}\right) \\
& =J_{m_{x z}}-m_{m}\left(x_{m}-x_{r f}\right)\left(z_{m}-z_{r f}\right)-m_{r w} x_{r f}\left(r_{r w}+z_{r f}\right)
\end{aligned}
$$

Finally, using the origin point $O$ as reference to the whole system, the previous moments of inertia and inertial products should be once again displaced and the final result regarding the global axis $x$ and $z$ are explicit on Eq. (A.12) to (A.14).

$$
\begin{aligned}
& J_{x x}=J_{r f_{x x}}+m_{r f} z_{r f}^{2}+J_{f f_{x x}}+m_{f f} z_{f f}^{2} \\
& J_{z z}=J_{r f_{z z}}+m_{r f} x_{r f}^{2}+J_{f f_{z z}}+m_{f f} x_{f f}^{2} \\
& J_{x z}=J_{r f_{x z}}-m_{r f} x_{r f} z_{r f}+J_{f f_{x z}}-m_{f f} x_{f f} z_{f f}
\end{aligned}
$$


A variable that constantly appears throughout the model is the perpendicular distance $u$ from the front frame center of mass to the steering axis, as illustrated in Figure A.1 and calculated in Eq. (A.15).

$$
\begin{aligned}
& \left|z_{f f}\right|=u / \sin (\epsilon)+w-x_{f f}+t / \tan (\epsilon) \\
& u=\left(x_{f f}-w-t\right) \cos (\epsilon)+\left|z_{f f}\right| \sin (\epsilon)
\end{aligned}
$$

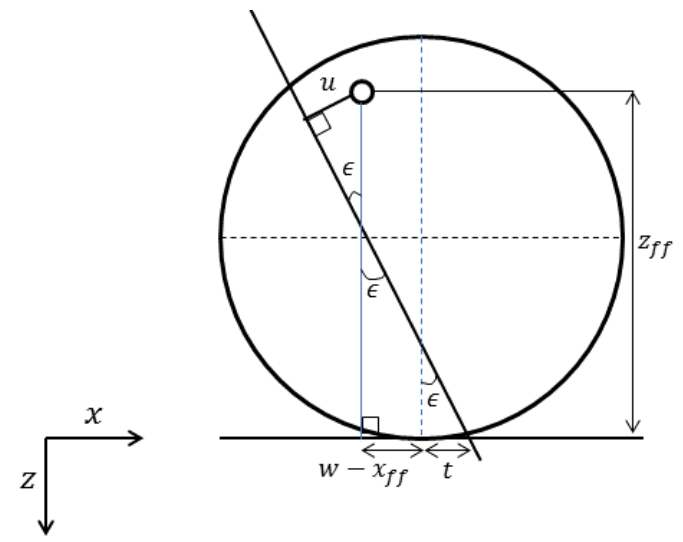

Figure A.1 - Perpendicular distance $u$ (Sharp ,1971).

Additionally, the front frame needs three extra inertia variables: the moment of inertia in respect to the steering axis and the inertia products relative to the points at which the steering axis (subscript $\epsilon$ ) intercepts the global axes $x$ and $z$. The inertia products provide torque information on one axis due to the angular acceleration of another, i.e., the gyroscopic effect. These new variables are described in Eq. (A.16) to (A.18) and Figure A.2 illustrates the steering axis.

$$
\begin{aligned}
& J_{\epsilon \epsilon}=m_{f f} u^{2}+J_{f f_{x x}} \sin (\epsilon)^{2}+2 J_{f f_{x z}} \sin (\epsilon) \cos (\epsilon)+J_{f f_{z z}} \cos (\epsilon)^{2} \\
& J_{\epsilon x}=-m_{f f} u z_{f f}+J_{f f_{x x}} \sin (\epsilon)+J_{f f_{x z}} \cos (\epsilon) \\
& J_{\epsilon z}=m_{f f} u x_{f f}+J_{f f_{x z}} \sin (\epsilon)+J_{f f_{z z}} \cos (\epsilon)
\end{aligned}
$$




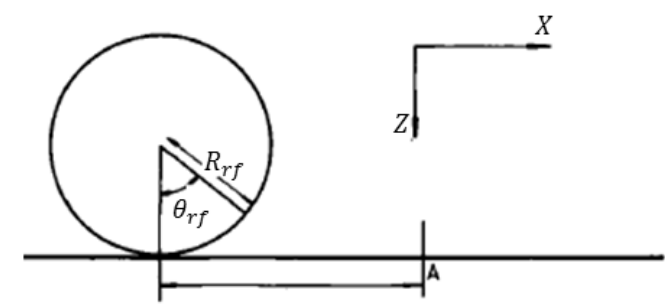

(a)

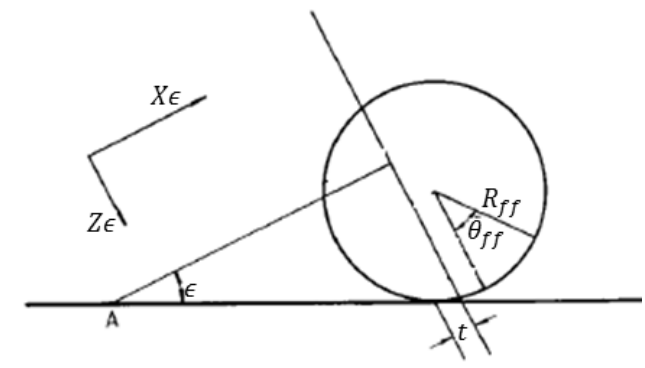

(b)

Figure A.2 - Axis in relation to frames: (a) Global axis at rear frame

(b) Steering axis at front frame (Sharp ,1971).

The last geometric variable to be defined is the ratio between the mechanical trail and the front wheel basis, that is, the perpendicular distance that the front wheel contact point is behind the steering axis, in Eq. (A.19).

$f=t \cos (\epsilon) / w$

Finally, the angular momentum of a body (Eq. (A.20)) can be defined by its linear speed and the ratio $S$ between its moment of inertia and angular radius of rotation. Therefore, the rear and front wheels angular momenta - $S_{r}$ and $S_{f}-$ with respect to the $y$ axis and its sum $\left(S_{t}\right)$ represent the gyrostatic effect coefficients, as described by Eq. (A.21). At last, Eq. (A.22) brings a frequently appearing static inertia term.

$L=J \omega=J(V / r)=S V$

$S_{f}=J_{f w_{y y}} / r_{f w} \quad S_{r}=J_{r w_{y y}} / r_{r w} \quad S_{t}=S_{f}+S_{r}$

$S_{u}=m_{f f} u+m_{t} x_{t} f$ 


\section{Appendix B. Traction motor characterization}

An interesting parameter to analyze when characterizing motors is to identify the torque applied with the command signal. For this test, it was used a dynamometer, built previously by undergraduate students to identify DC motors parameters. It has a known inertial load (wood disc) with two diametrically opposite magnets coupled with a Hall effect sensor, working as a tachometer (Figure B.1). Using a National Instruments data acquisition board, by monitoring the number of peaks (or half rotations) within a window of 0.3 seconds it is possible to calculate the angular velocity of the disc, in rad/s (Eq. (B.1)).

$\omega(t)=(2 \pi / \Delta t) \times($ Number of peaks/Number of magnets $)$
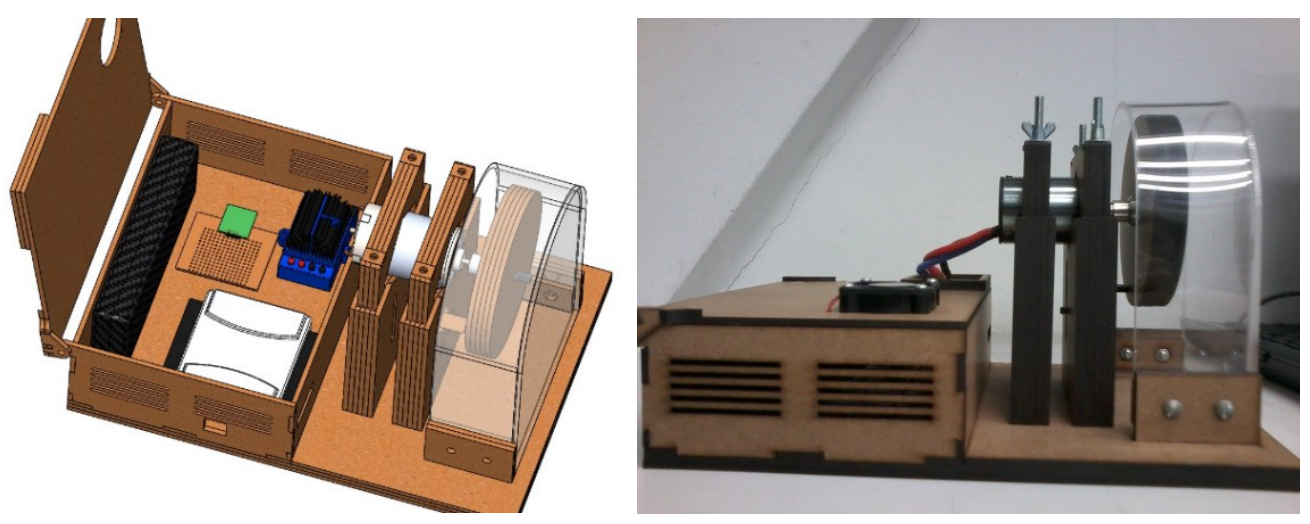

Figure B.1 -Dynamometer for electric motors.

The tests consisted of increasing and decreasing the command signal with constant amplitude variations, equal to $10 \%, 20 \%$ and $30 \%$ of the maximum power. Through the experiment, it was noted no speed increase after $70 \%$ of the signal, due to an ESC limitation; the subsequent tests were adjusted to ignore this range of command signal. The disc has a mass of $120 \mathrm{~g}$ and radius of $5 \mathrm{~cm}$ - which results in an load of $1.510^{-4} \mathrm{~N} . \mathrm{m}$ - which is six times smaller than the rear wheel moment of inertia in the $y$ axis. The motor torque has a linear relation with the disc angular acceleration and inertial load, as explicit in Eq. (B.2). 
$\tau=J \dot{\omega}$
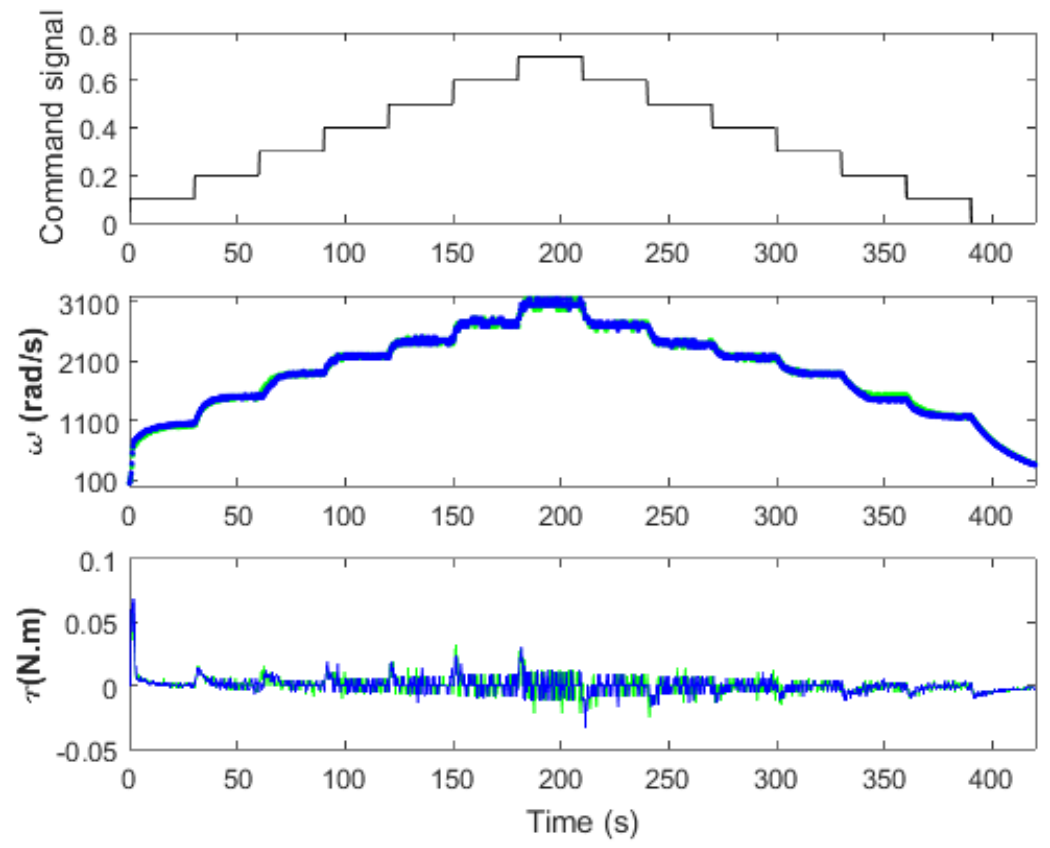

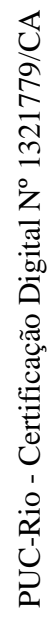

Figure B.2 -Dynamometer test with $10 \%$ step.
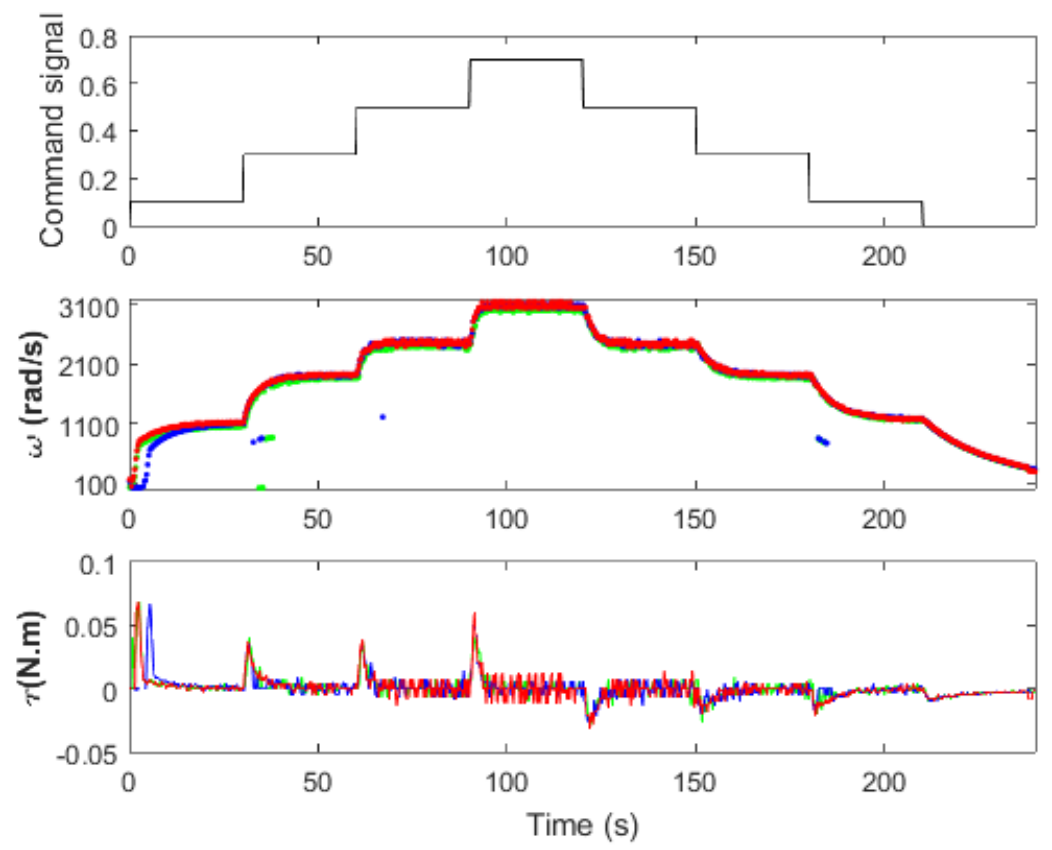

Figure B.3 -Dynamometer test with $20 \%$ step. 

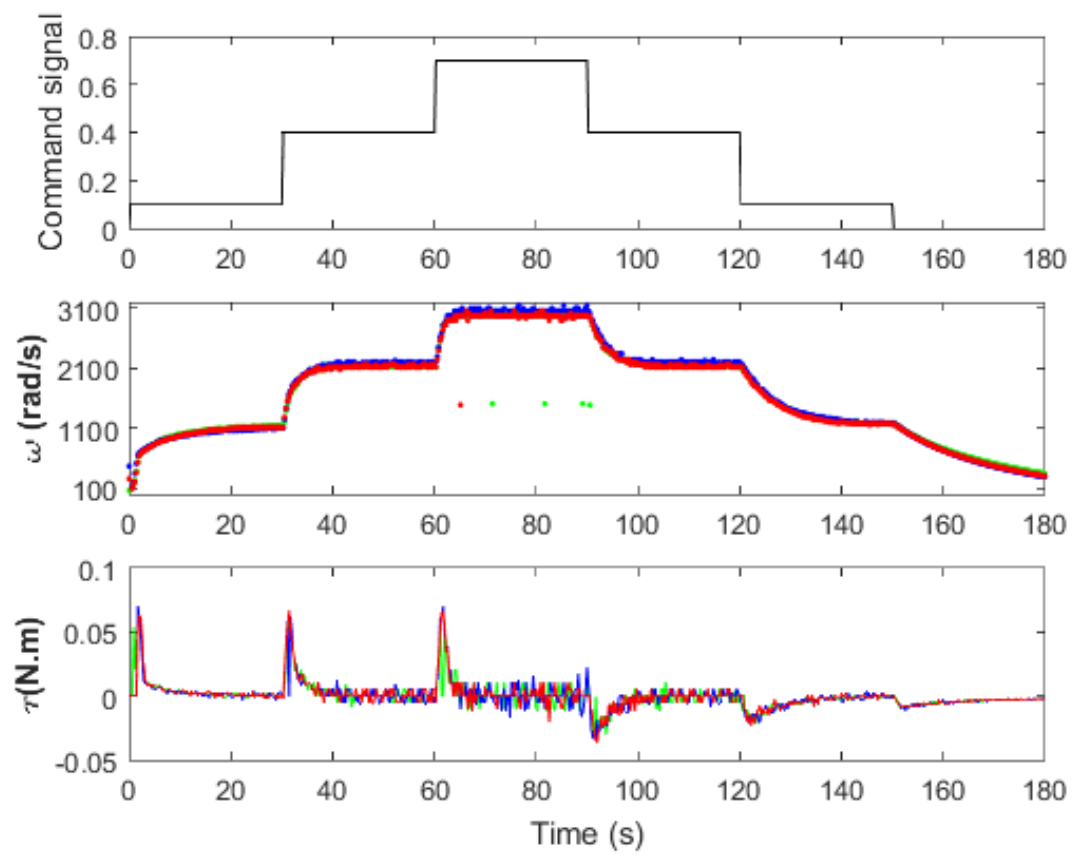

Figure B.4 -Dynamometer test with $\mathbf{3 0 \%}$ step.

With this experiment, it was possible to analyze that the maximum step between command signals is $30 \%$, since higher amplitudes cause a battery overcurrent; additionally, for the same reason, the motor can only be started at a maximum of $20 \%$. Finally, the maximum torque for the motor is of 0.07 N.m. 


\section{Appendix C. IMU measurer}

To validate the measurements from the inertial measurement unit, it was used an IMU measurer, developed by Schuback (2014). The device consists of an electrical DC motor that spins a board clockwise, connected to an encoder; with different voltage levels, the equipment produces varied angular speeds, which results in different centripetal accelerations. Figure C.1 brings the device's concept in SolidWorks and the built result.
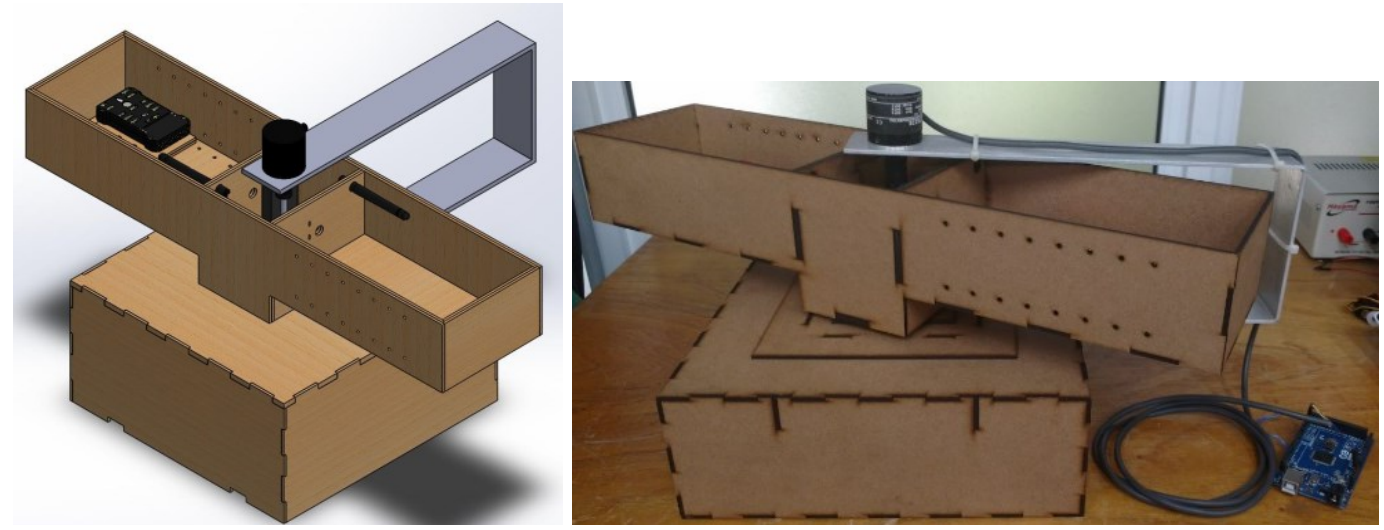

Figure C.1 - IMU measurer.

The centripetal acceleration exercised over the IMU has a direct relation between the system's angular speed and the distance to the rotational axis (Eq. (C.1)). The angular speed is measured by both the IMU's gyrometer and the incremental encoder, whose pulses are read and interpreted as angular rate by an Arduino, which in turn transmits the data to a computer via serial communication. The Pixhawk microcontroller saves the IMU data obtained during the tests on a $S D$ card for later analysis and also sends the signal via serial communication. A Labview routine was responsible for receiving data from both devices and providing real-time graphs.

$a=r \omega^{2}$ 
The first test consisted of applying three different voltage levels to the motor (4, 8 and 12V) and analyzing the measurements made by the encoder and the IMU. The test was repeated with the IMU spinning at three different axis; Figure C.3 to Figure C.7 illustrates the results to, respectively, the $\mathrm{x}, \mathrm{y}$ and $z$ axis in the same direction as the tangential velocity.
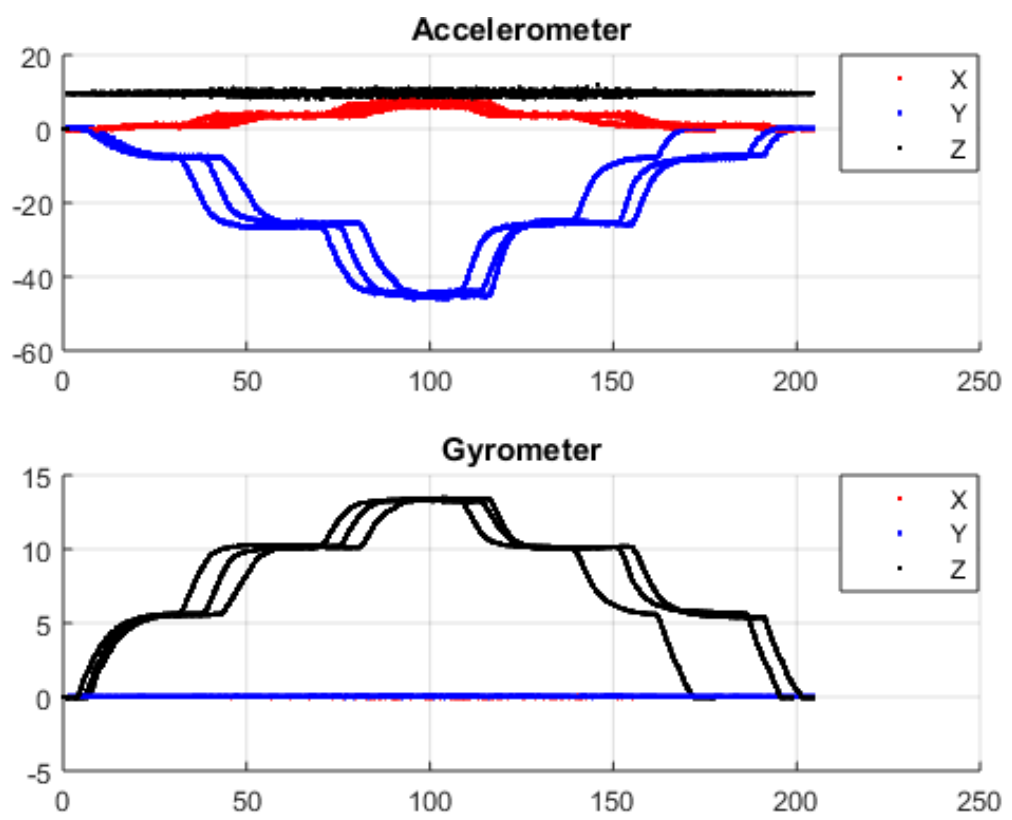

Figure C.2 - Accelerations with planar position on the $x$ axis.
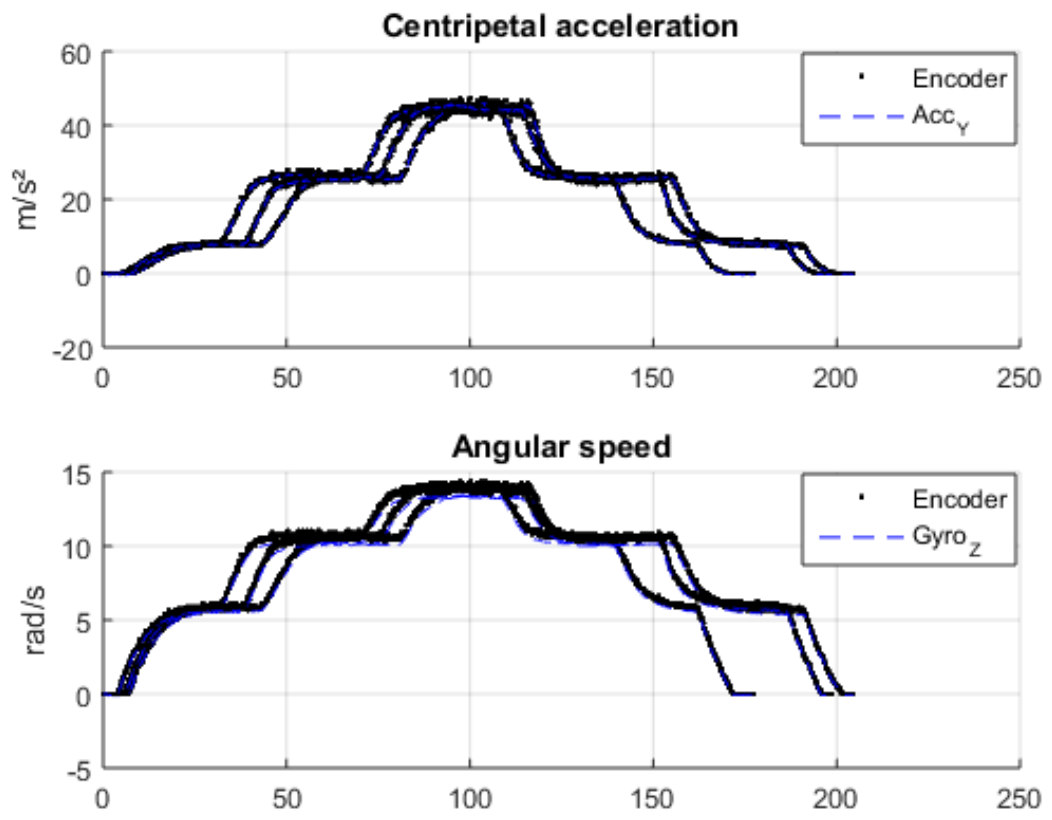

Figure C.3 - Comparison with encoder measurements, $x$ axis. 

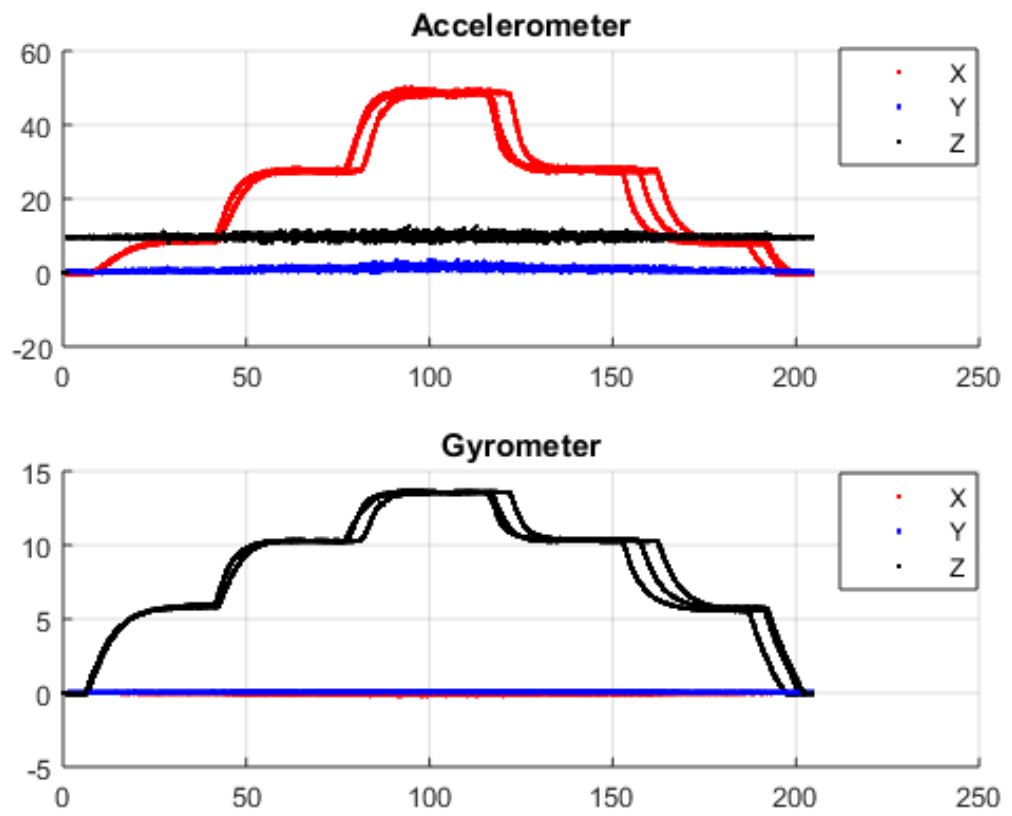

Figure C.4 - Accelerations with planar position on the $y$ axis.
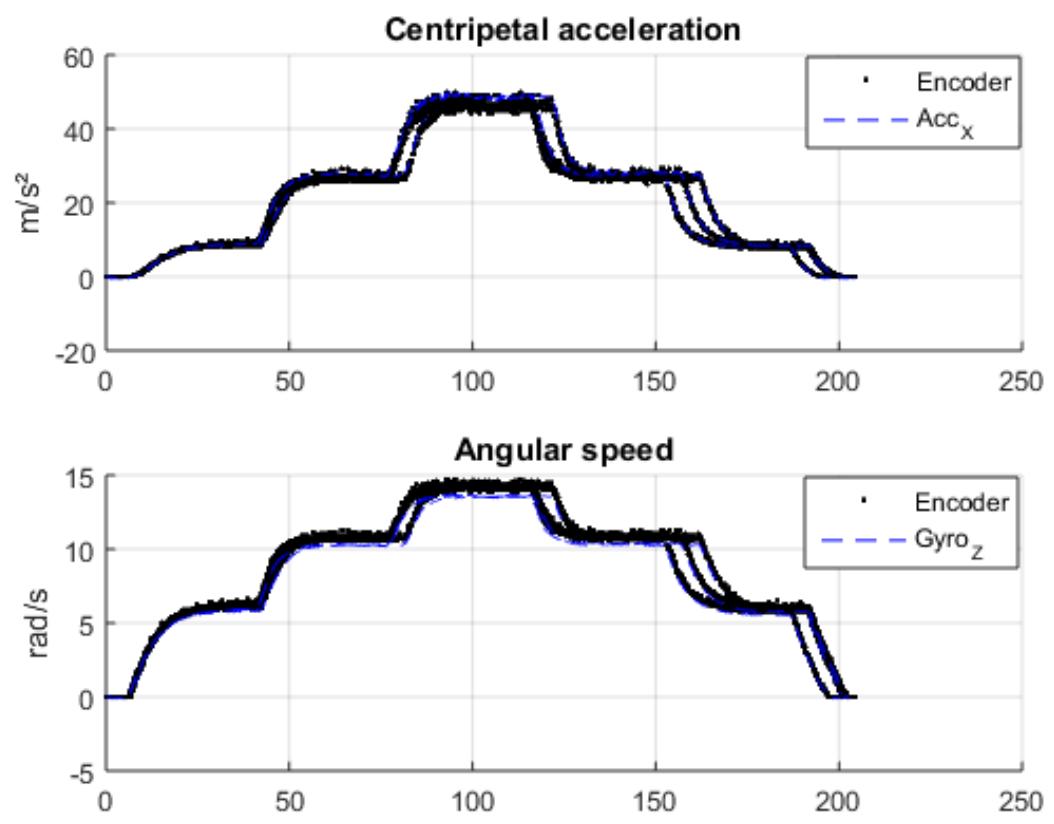

Figure C.5 - Comparison with encoder measurements, $y$ axis. 

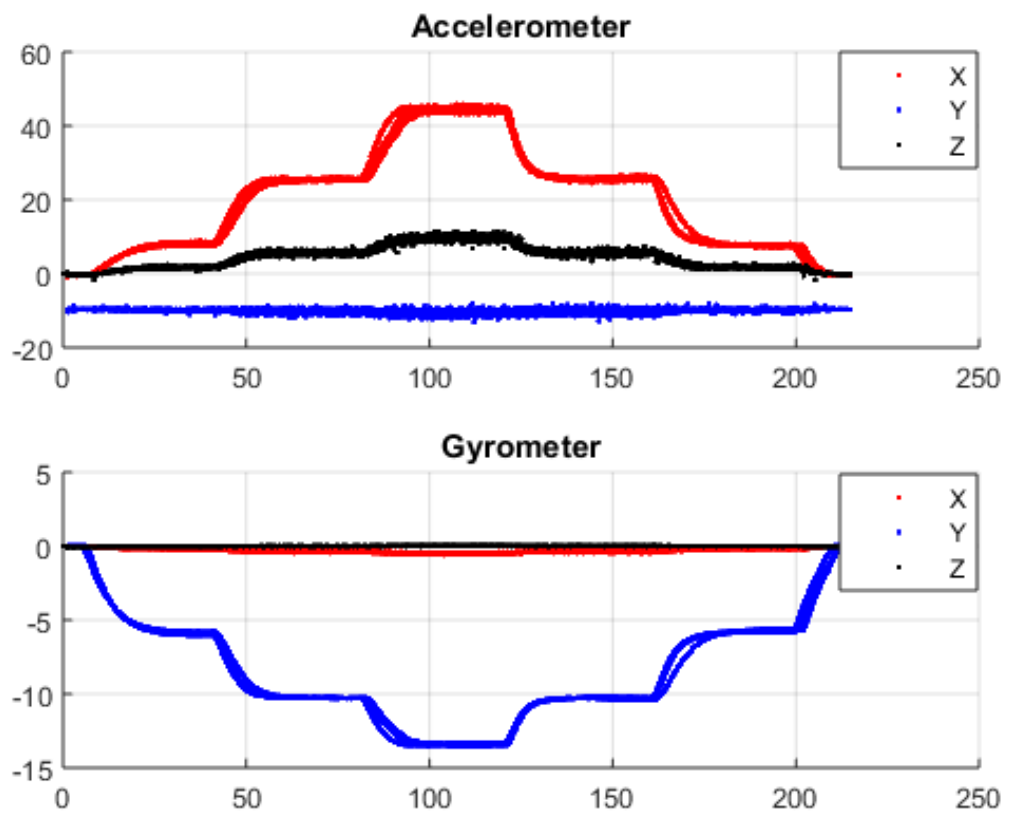

Figure C.6 - Accelerations with planar position on the $z$ axis.
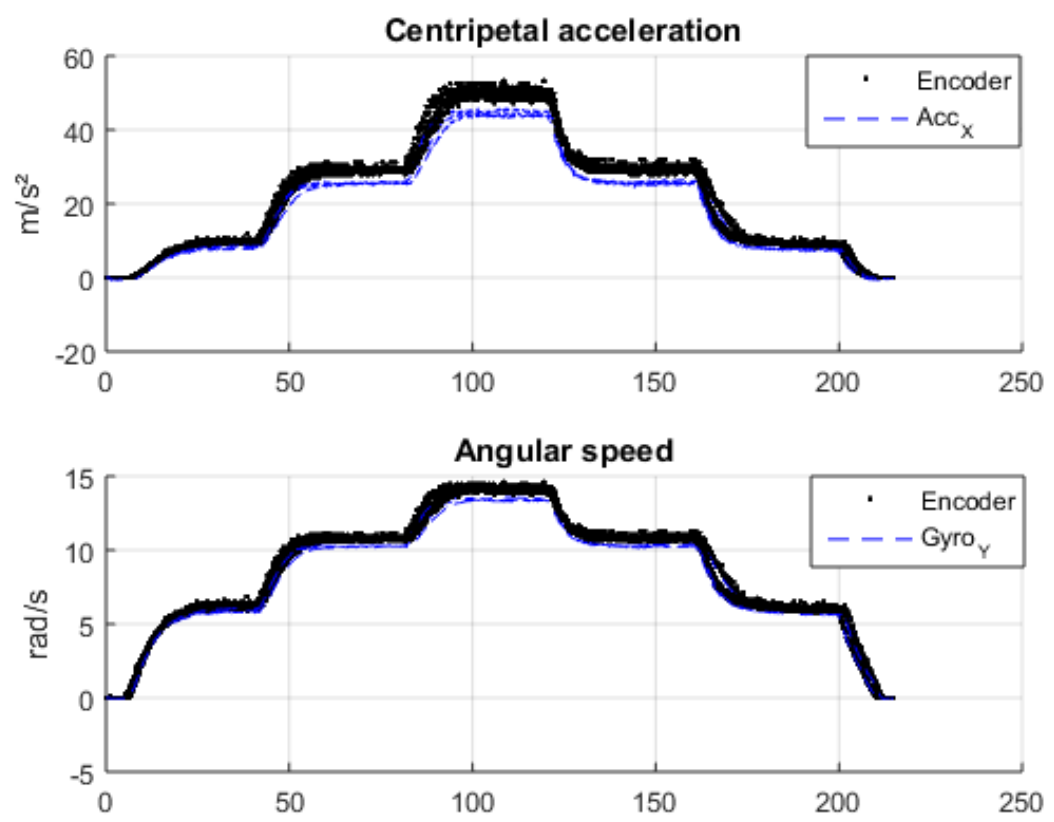

Figure C.7 - Comparison with encoder measurements, $z$ axis. 
The second test consisted of repeating the previous one, with an additional inclination to the IMU, simulating a constant roll angle to the system (Figure C.8). The IMU was fixed with a 15 degrees ( $0.26 \mathrm{rad}$ ) inclination and the new relations between centripetal acceleration and angular speed are defined in Eq. (C.2) and (C.3). Figure C.10 present the results, considering the $x$ axis positive in the direction of the tangential speed, $y$ axis positive in the centripetal direction and the $z$ axis, positive in the gravitational direction.

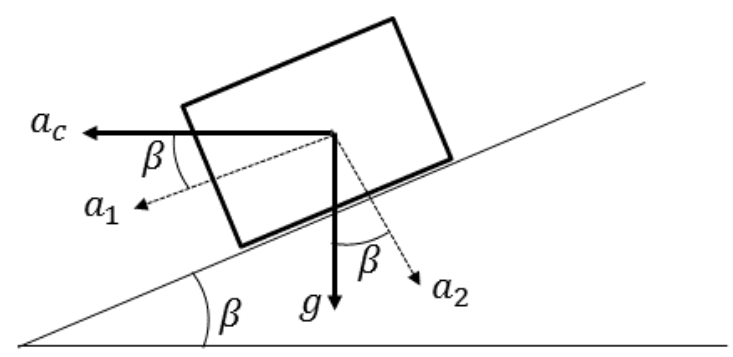

Figure C.8 - Inclined IMU position.

$a_{c}=r \omega^{2}=a_{1} \cos (\beta)-a_{2} \sin (\beta)$

$\omega=\omega_{1} \sin (\beta)+\omega_{2} \cos (\beta)$
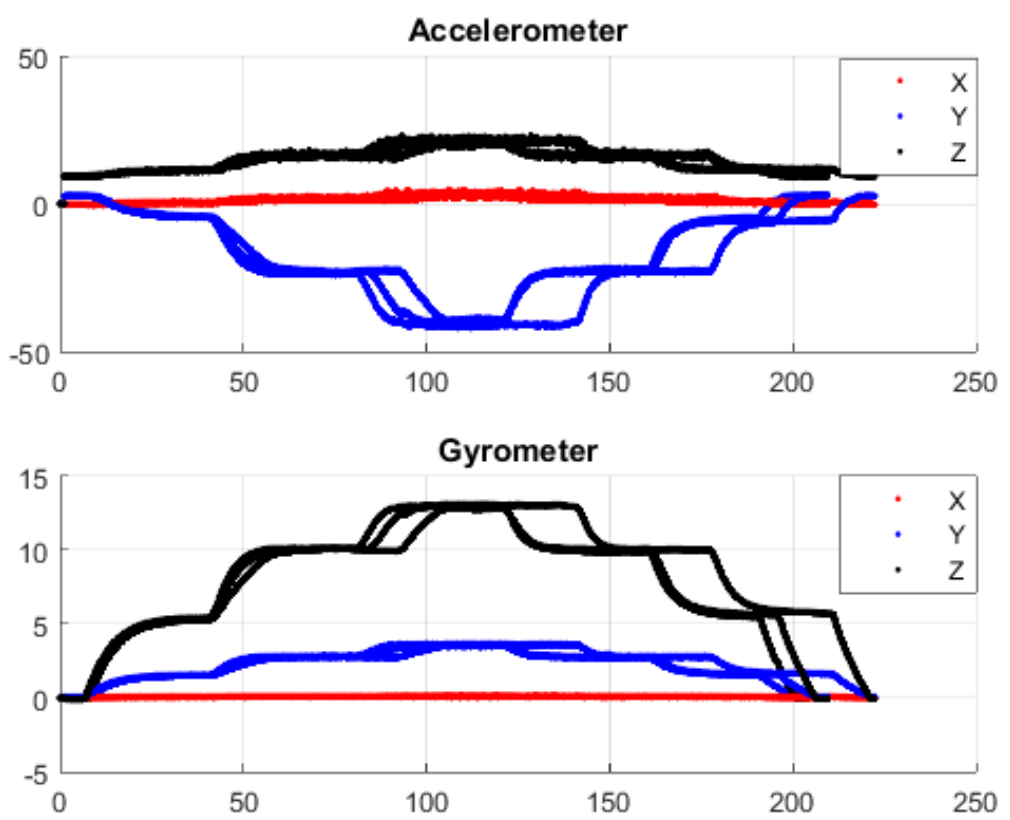

Figure C.9 - Accelerations with leaning position on the $x$ axis. 

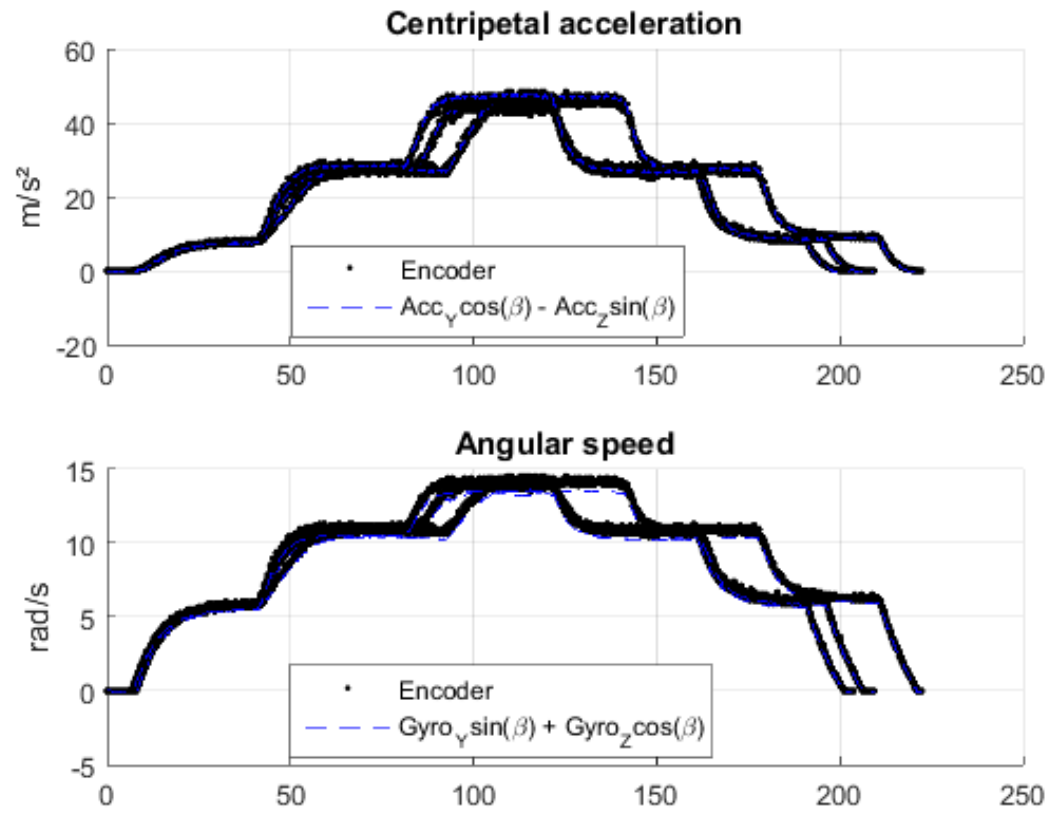

Figure C.10 - Comparison with encoder measurements, leaning position on the $x$ axis. 


\section{Appendix D. Programs}

The following programs were used during this thesis and can be obtained in the attached CD. The Matlab version used was R2015a; the Labview license was 2013; the Arduino ide was the version 1.6.9 and the Pixhawk firmware was version 1.4 .

\section{Chapter 2}

\section{Matlab}

Center_of mass.m

TractionSystem_Dynamometer.m

TractionSystem_Embedded.m/ calcula_w.m / dvx_dt.slx

SteeringSystem.m/ steering_simulation.slx

\section{Labview}

Arduino_serial.vi

Main_Motor_Moto.vi

Matlab_Plot.vi

Pwm.vi

Px4_Start.vi/ Px4_Stop.vi

\section{Arduino}

Read_Hall_RPM_Sensor.ino

\section{Chapter 3}

Model_analysis.m

\section{Chapter 4}

Control_main.m

Error_prediction.m/ error_prediction.slx

LQR_feedback.m/lqr_feedback.slx

Observer_feedback.m/ observer_feedback.slx

Output_feedback.m/ output_feedback.slx 
Path_comparison.m

Path_preview.m/ path_preview.slx

Roll_review.m/ roll_preview.slx

State_feedback.m/ state_feedback.slx

\section{Chapter 5}

Filter_part1.m

Filter_part2.m

Kalman_loop.m

Motorcycle_IMU.m

Sensor_AccGiro.m

Simulation_with_WhiteNoise.m

\section{Chapter 6}

\section{Matlab}

Read_Labview.m

\section{Labview}

Arduino_Read.vi

Arduino_Send.vi

Arduino_Write.vi

Draw_bike.vi

PlataformaAtuada_Main.vi

Px4_Read.vi / Px4_Start.vi/ Px4_Stop.vi

Simulacao_Teste2.txt

\section{Arduino}

Arduino_Plataforma_Atuada.ino

\section{Pixhawk}

rc.txt

arduino_publisher.c

kalman_orientation.c/ kalman_orientation.h

kalman_publisher.c

matrix_manipulation.c/ matrix_manipulation.h

motorcycle.c

motorcycle_parameter.c/ motorcycle_parameter.h 